\title{
Oxazines: A New Class Of Second-Order Nonlinear Optical Switches - Supporting Information
}

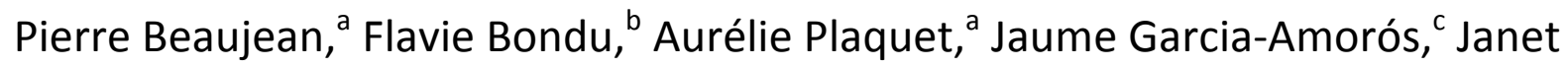
Cusido, ${ }^{\mathrm{c}}$ Françisco M. Raymo, ${ }^{\mathrm{c},{ }^{*}}$ Frédéric Castet, ${ }^{\mathrm{b},{ }^{*}}$ Vincent Rodriguez, ${ }^{\mathrm{b},{ }^{*}}$ and Benoît Champagne ${ }^{a,{ }^{*}}$

a University of Namur, Laboratoire de Chimie Théorique, Unité de Chimie Physique Théorique et Structurale, rue de Bruxelles, 61, B-5000 Namur, Belgium

${ }^{b}$ Université de Bordeaux, Institut des Sciences Moléculaires (ISM), UMR 5255 CNRS, Cours de la Libération 351, F-33405 Talence Cedex, France

c Laboratory for Molecular Photonics, Department of Chemistry, University of Miami, 1301 Memorial Drive, Coral Gables, FL 33146-0431, USA

\begin{tabular}{|c|c|c|c|}
\hline & c & a & $\mathbf{R}^{\mathbf{2}}$ \\
\hline M06-2X & 0.985 & 0.415 & 0.979 \\
\hline
\end{tabular}

Table S1. Correlation $\left[\Delta \mathrm{E}^{\mathrm{TDDFT}}=\mathrm{c} \times \Delta \mathrm{E}^{\mathrm{Exp} .}+\right.$ a] between experimental maximum absorption energies and TDDFT/M06-2X vertical excitation energies for the lowest-energy band of the UV/vis absorption spectra of compounds 3, 4, 11, and $\mathbf{1 2}$ in their closed and protonated open forms. IEFPCM was used to describe the solvent (chloroform) effects. The $6-311+G(d)$ basis set was employed. 


\begin{tabular}{|c|c|c|c|c|c|c|}
\hline & \multicolumn{3}{|c|}{ Closed (a) } & \multicolumn{3}{c|}{ Open $\left(\mathrm{b}^{+}\right)$} \\
\hline & $\Delta \mathrm{E}(\mathrm{eV})$ & $\Gamma\left(\mathrm{cm}^{-1}\right)$ & $\mathrm{F}$ & $\Delta \mathrm{E}(\mathrm{eV})$ & $\Gamma\left(\mathrm{cm}^{-1}\right)$ & $\mathrm{F}$ \\
\hline $\mathbf{3}$ & 4.25 & 6320 & 1.31 & 2.78 & 4160 & 2.07 \\
$\mathbf{4}$ & 3.90 & 5390 & 1.40 & 2.94 & 4490 & 1.88 \\
$\mathbf{1 1}$ & 3.04 & 3730 & 1.91 & 2.03 & 2070 & 1.97 \\
$\mathbf{1 2}$ & 4.29 & 4780 & 1.32 & 2.27 & 2530 & 3.05 \\
\hline
\end{tabular}

Table S2. Characteristics of the main low-energy band of the UV/Vis absorption spectra of the closed and protonated open forms in chloroform [Energies of the maximum of absorption $(\Delta E)$, full width at half maximum, $\Gamma]$, and frequency dispersion factor, $F=\beta_{\text {HRS }}(1064) / \beta_{\text {HRS }}(1907)$.

\begin{tabular}{|c|c|c|c|c|c|c|c|c|c|c|}
\hline & \multicolumn{5}{|c|}{ Closed (a) } & \multicolumn{3}{c|}{ Open (b+ ${ }^{+}$} & \multicolumn{3}{c|}{ Open (b) } \\
\hline & $\beta^{\mathrm{MP2}}(0)$ & $\beta^{\mathrm{CPHF}}(0)$ & $\beta^{\mathrm{TDHF}}(1907)$ & $\beta^{\mathrm{MP} 2}(1907)$ & $\beta^{\mathrm{MP2}}(0)$ & $\beta^{\mathrm{CPHF}}(0)$ & $\beta^{\mathrm{TDHF}}(1907)$ & $\beta^{\mathrm{MP} 2}(1907)$ & $\beta^{\mathrm{MP2}}(0)$ & $\beta^{\mathrm{CPHF}}(0)$ \\
\hline $\mathbf{3}$ & 1451 & 803 & 725 & $\mathbf{1 3 0 8}$ & 5391 & 2433 & 2137 & $\mathbf{4 7 3 4}$ & 3501 & 7831 \\
$\mathbf{4}$ & 1418 & 771 & 697 & $\mathbf{1 2 8 3}$ & 4558 & 1928 & 1666 & $\mathbf{3 9 3 9}$ & 2968 & 6556 \\
$\mathbf{1 1}$ & 6723 & 3001 & 2865 & $\mathbf{6 4 1 6}$ & 32367 & 12670 & 12365 & $\mathbf{3 1 5 8 8}$ & 11487 & 31589 \\
$\mathbf{1 2}$ & 2252 & 1242 & 1154 & $\mathbf{2 0 9 2}$ & 18184 & 7955 & 7549 & $\mathbf{1 7 2 5 7}$ & 7271 & 16782 \\
\hline
\end{tabular}

Table S3. First hyperpolarizabilities (a.u.) of the protonated open and closed forms as determined at different levels of approximation within the IEFPCM solvation model (solvent = chloroform) with the $6-311+G(d)$ basis set together with the estimates of the MP2 dynamic first hyperpolarizabilities calculated using the multiplicative scheme. The last two columns report the static MP2 and HF first hyperpolarizabilities of the zwitterionic open forms evaluated within the IEFPCM solvation model (solvent = chloroform) and the $6-311+G(d)$ basis set. 

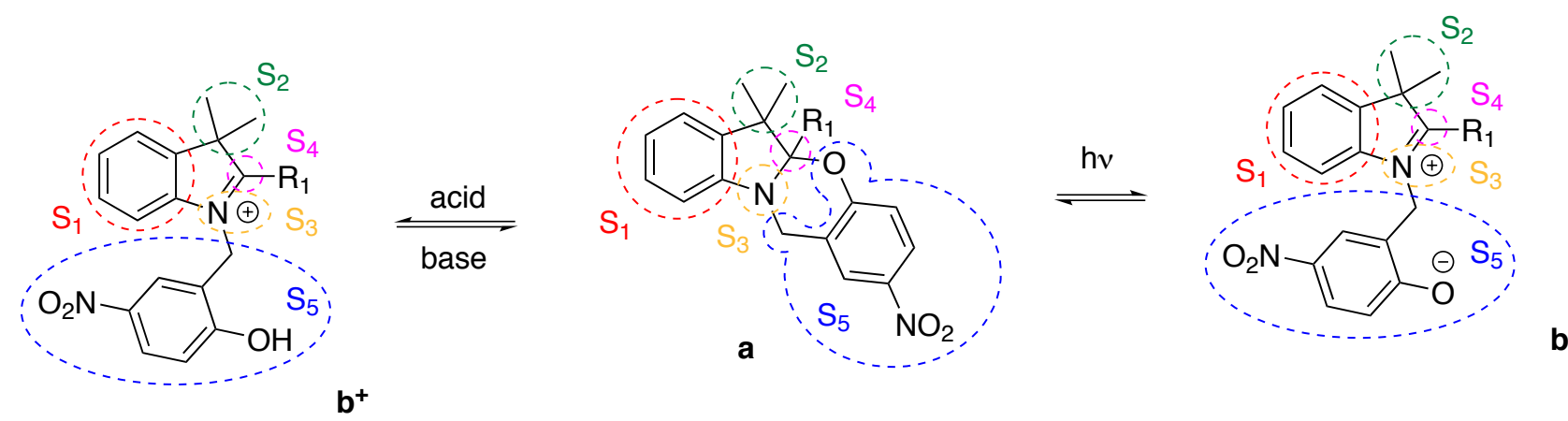

\begin{tabular}{|c|c|c|c|c|c|c|c|c|c|c|}
\hline & $3 a$ & $4 a$ & $5 a$ & $6 a$ & $7 a$ & $8 a$ & $9 a$ & $10 a$ & $11 a$ & $12 a$ \\
\hline$S_{1}$ & 0.261 & 0.259 & 0.257 & 0.261 & 0.246 & 0.252 & 0.267 & 0.264 & 0.253 & 0.256 \\
\hline $\mathrm{S}_{2}$ & -0.022 & -0.023 & -0.022 & -0.001 & -0.029 & -0.027 & -0.011 & -0.013 & -0.023 & -0.027 \\
\hline $\mathrm{S}_{3}$ & -0.411 & -0.411 & -0.410 & -0.417 & -0.408 & -0.407 & -0.408 & -0.408 & -0.406 & -0.410 \\
\hline $\mathrm{S}_{4}$ & 0.166 & 0.166 & 0.166 & 0.132 & 0.039 & 0.167 & 0.164 & 0.164 & 0.181 & 0.165 \\
\hline $\mathrm{S}_{5}$ & -0.118 & -0.118 & -0.120 & -0.102 & -0.117 & -0.128 & -0.109 & -0.113 & -0.132 & -0.121 \\
\hline \multirow[t]{2}{*}{$\mathrm{R}_{1}$} & 0.124 & 0.127 & 0.129 & 0.126 & 0.269 & 0.144 & 0.097 & 0.105 & 0.127 & 0.136 \\
\hline & & $4 b$ & $5 b$ & $6 b$ & $7 b$ & $8 b$ & $9 b$ & $10 \mathrm{~b}$ & $11 b$ & $12 b$ \\
\hline$S_{1}$ & 0.435 & 0.437 & 0.415 & 0.471 & 0.480 & 0.404 & 0.471 & 0.463 & 0.44 & 0.435 \\
\hline $\mathrm{S}_{2}$ & -0.016 & -0.014 & -0.022 & -0.020 & -0.006 & -0.026 & -0.002 & -0.005 & -0.021 & -0.016 \\
\hline $\mathrm{S}_{3}$ & -0.441 & -0.440 & -0.448 & -0.364 & -0.319 & -0.453 & -0.429 & -0.431 & -0.454 & -0.460 \\
\hline $\mathrm{S}_{4}$ & 0.435 & 0.437 & 0.442 & 0.330 & 0.143 & 0.439 & 0.453 & 0.452 & 0.454 & 0.444 \\
\hline $\mathrm{S}_{5}$ & -0.636 & -0.629 & -0.652 & -0.615 & -0.612 & -0.66 & -0.609 & -0.617 & -0.694 & -0.702 \\
\hline \multirow[t]{2}{*}{$\mathrm{R}_{1}$} & 0.223 & 0.208 & 0.266 & 0.198 & 0.314 & 0.296 & 0.117 & 0.139 & 0.274 & 0.299 \\
\hline & $3 b^{+}$ & $4 b^{+}$ & $5 b^{+}$ & $6 b^{+}$ & $7 b^{+}$ & $8 b^{+}$ & $9 b^{+}$ & $10 b^{+}$ & $11 b^{+}$ & $12 b^{+}$ \\
\hline $\mathrm{S}_{1}$ & 0.468 & 0.468 & 0.437 & 0.520 & 0.533 & 0.430 & 0.505 & 0.496 & 0.455 & 0.455 \\
\hline $\mathrm{S}_{2}$ & 0.002 & 0.004 & -0.008 & 0.008 & 0.021 & -0.011 & 0.017 & 0.014 & -0.006 & -0.002 \\
\hline $\mathrm{S}_{3}$ & -0.467 & -0.454 & -0.477 & -0.368 & -0.333 & -0.478 & -0.451 & -0.452 & -0.478 & -0.475 \\
\hline $\mathrm{S}_{4}$ & 0.455 & 0.457 & 0.456 & 0.321 & 0.154 & 0.461 & 0.475 & 0.479 & 0.454 & 0.440 \\
\hline $\mathrm{S}_{5}$ & 0.229 & 0.241 & 0.203 & 0.288 & 0.298 & 0.202 & 0.263 & 0.241 & 0.217 & 0.138 \\
\hline $\mathrm{R}_{1}$ & 0.313 & 0.284 & 0.389 & 0.231 & 0.327 & 0.395 & 0.192 & 0.223 & 0.358 & 0.445 \\
\hline
\end{tabular}

Table S4. Mulliken charges on the fragments of the oxazine compounds 3-12 in their closed (a) as well as photochemically- (b) and acidly-open $\left(\mathbf{b}^{+}\right)$forms as determined at the IEF-PCM/M06/6$311 \mathrm{G}(\mathrm{d})$ level (in chloroform). 


\begin{tabular}{|c|c|c|c|c|c|c|c|c|c|c|c|c|}
\hline & \multicolumn{4}{|c|}{ Closed (a) } & \multicolumn{4}{|c|}{ open (b) } & \multicolumn{4}{|c|}{ open $\left(\mathbf{b}^{+}\right)$} \\
\hline & \multicolumn{2}{|c|}{ static } & \multicolumn{2}{|c|}{$1064 \mathrm{~nm}$} & \multicolumn{2}{|c|}{ static } & \multicolumn{2}{|c|}{$1064 \mathrm{~nm}$} & \multicolumn{2}{|c|}{ static } & \multicolumn{2}{|c|}{$1064 \mathrm{~nm}$} \\
\hline & $\beta_{\mathrm{HRS}}$ & $\mathrm{DR}$ & $\beta_{\mathrm{HRS}}$ & $\mathrm{DR}$ & $\beta_{\mathrm{HRS}}$ & $\mathrm{DR}$ & $\beta_{\mathrm{HRS}}$ & DR & $\beta_{\mathrm{HRS}}$ & $\mathrm{DR}$ & $\beta_{\mathrm{HRS}}$ & DR \\
\hline 1 & 1239 & 4.65 & 1442 & 4.79 & 8003 & 4.21 & 13375 & 4.57 & 8868 & 4.36 & 15044 & 4.69 \\
\hline 2 & 577 & 2.94 & 661 & 3.19 & 4534 & 2.81 & 9330 & 3.65 & 819 & 2.51 & 972 & 2.69 \\
\hline 3 & 803 & 3.57 & 902 & 3.91 & 3501 & 5.41 & 4770 & 5.93 & 2521 & 4.53 & 3522 & 4.83 \\
\hline 4 & 771 & 3.55 & 862 & 3.86 & 2968 & 5.33 & 3681 & 6.01 & 1928 & 4.43 & 2405 & 4.75 \\
\hline 5 & 1386 & 3.48 & 1764 & 3.78 & 10860 & 5.04 & 20298 & 5.17 & 11603 & 4.59 & 22927 & 4.84 \\
\hline 6 & 697 & 3.01 & 790 & 3.30 & 1656 & 3.38 & 1802 & 3.64 & 413 & 3.58 & 408 & 3.87 \\
\hline 7 & 718 & 2.94 & 796 & 3.23 & 1738 & 3.41 & 1959 & 3.68 & 421 & 3.60 & 425 & 3.79 \\
\hline 8 & 1648 & 4.78 & 1947 & 5.07 & 9200 & 5.11 & 15215 & 5.28 & 8517 & 4.47 & 14285 & 4.76 \\
\hline 9 & 1038 & 2.58 & 1310 & 2.92 & 1658 & 2.84 & 1830 & 2.70 & 604 & 2.63 & 814 & 2.93 \\
\hline 10 & 853 & 2.58 & 1015 & 2.81 & 2011 & 4.15 & 2340 & 4.74 & 1057 & 4.18 & 1239 & 4.60 \\
\hline 11 & 3001 & 4.21 & 3929 & 4.62 & 11487 & 4.27 & 21392 & 4.67 & 12670 & 4.18 & 24891 & 4.55 \\
\hline 12 & 1242 & 4.26 & 1437 & 4.58 & 7271 & 4.55 & 11652 & 4.89 & 7955 & 4.35 & 13499 & 4.71 \\
\hline
\end{tabular}

Table S5: Static and dynamic $(1064 \mathrm{~nm})$ hyper-Rayleigh scattering first hyperpolarizabilities $\left(\beta_{H R S}\right.$, a.u.) and depolarization ratios (DR) of the closed and open forms of compounds 1-12, obtained at the IEF-PCM/TDHF/6-311+G(d) level (in chloroform).

\begin{tabular}{cccccc}
\hline \hline & \multicolumn{2}{c}{$\beta_{\text {HRS }}(\mathbf{b}) / \beta_{\text {HRS }}(\mathbf{a})$} & & \multicolumn{2}{c}{$\beta_{\text {HRS }}\left(\mathbf{b}^{+}\right) / \beta_{\text {HRS }}(\mathbf{a})$} \\
\cline { 2 - 3 } \cline { 5 - 6 } & Static & $1064 \mathrm{~nm}$ & & Static & $1064 \mathrm{~nm}$ \\
\hline $\mathbf{1}$ & 6.46 & 9.28 & & 7.16 & 10.43 \\
$\mathbf{2}$ & 7.86 & 14.12 & & 1.42 & 1.47 \\
$\mathbf{3}$ & 4.36 & 5.29 & & 3.14 & 3.91 \\
$\mathbf{4}$ & 3.85 & 4.27 & & 2.50 & 2.79 \\
$\mathbf{5}$ & 7.84 & 11.51 & & 8.37 & 13.00 \\
$\mathbf{6}$ & 2.37 & 2.28 & & 0.59 & 0.52 \\
$\mathbf{7}$ & 2.42 & 2.46 & & 0.59 & 0.53 \\
$\mathbf{8}$ & 5.58 & 7.81 & & 5.17 & 7.34 \\
$\mathbf{9}$ & 1.60 & 1.40 & & 0.58 & 0.62 \\
$\mathbf{1 0}$ & 2.36 & 2.31 & & 1.24 & 1.22 \\
$\mathbf{1 1}$ & 3.83 & 5.44 & & 4.22 & 6.33 \\
$\mathbf{1 2}$ & 5.85 & 8.11 & 6.40 & 9.39 \\
\hline \hline
\end{tabular}

Table S6: Static and dynamic (1064nm) $\beta_{\mathrm{HRS}}$ (open)/ $\beta_{\mathrm{HRS}}$ (closed) contrast ratios for the photo- and acido-triggered opening of compounds 1-12, as determined at the IEF-PCM/TDHF/6-311+G(d) level (in chloroform). 

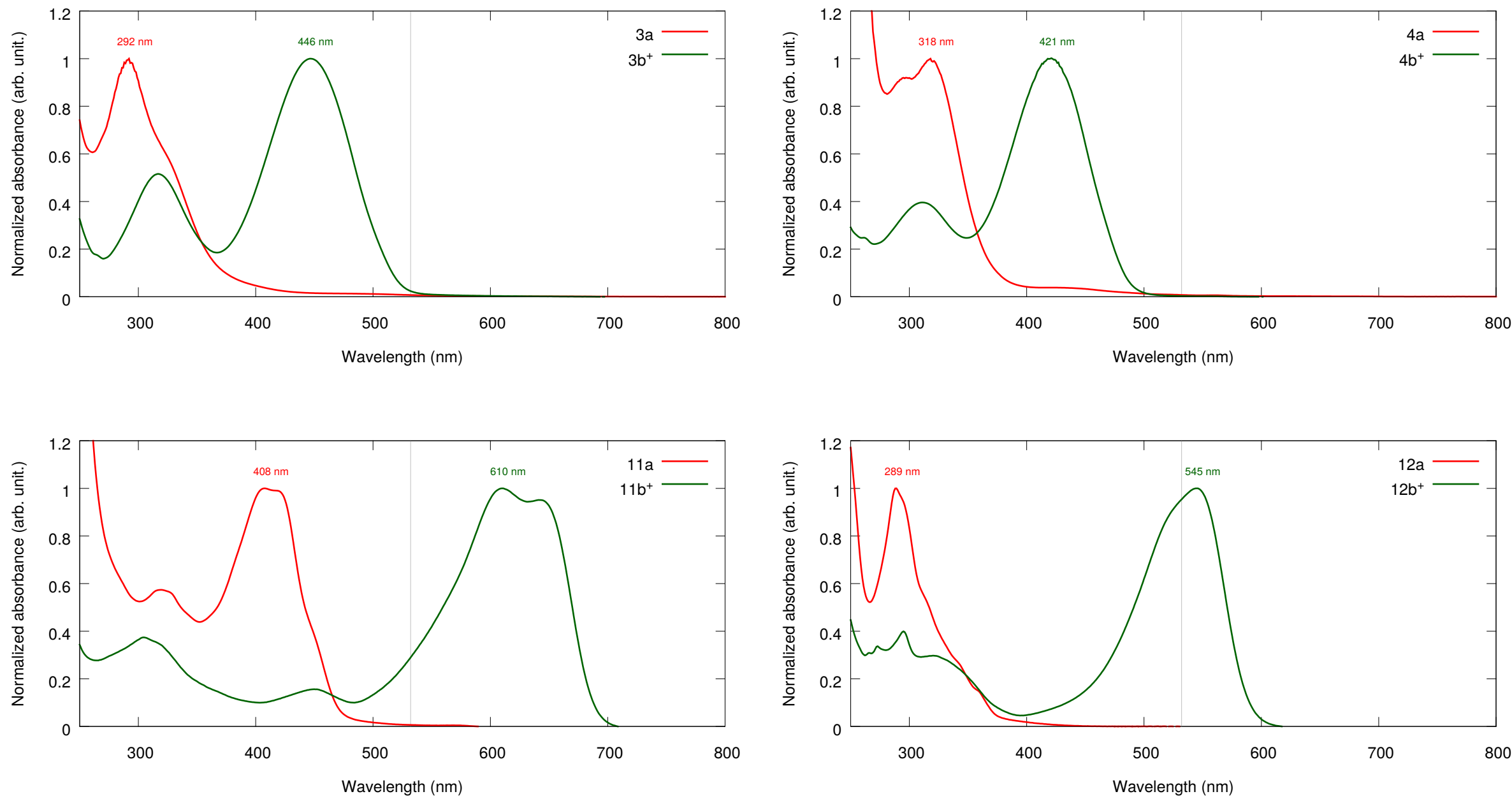

Figure S1. Experimental UV/vis absorption spectra of the closed and protonated open forms of 3, 4, 11, and 12 recorded in chloroform. The vertical grey line at $532 \mathrm{~nm}$ highlights the SHG signal wavelength. 

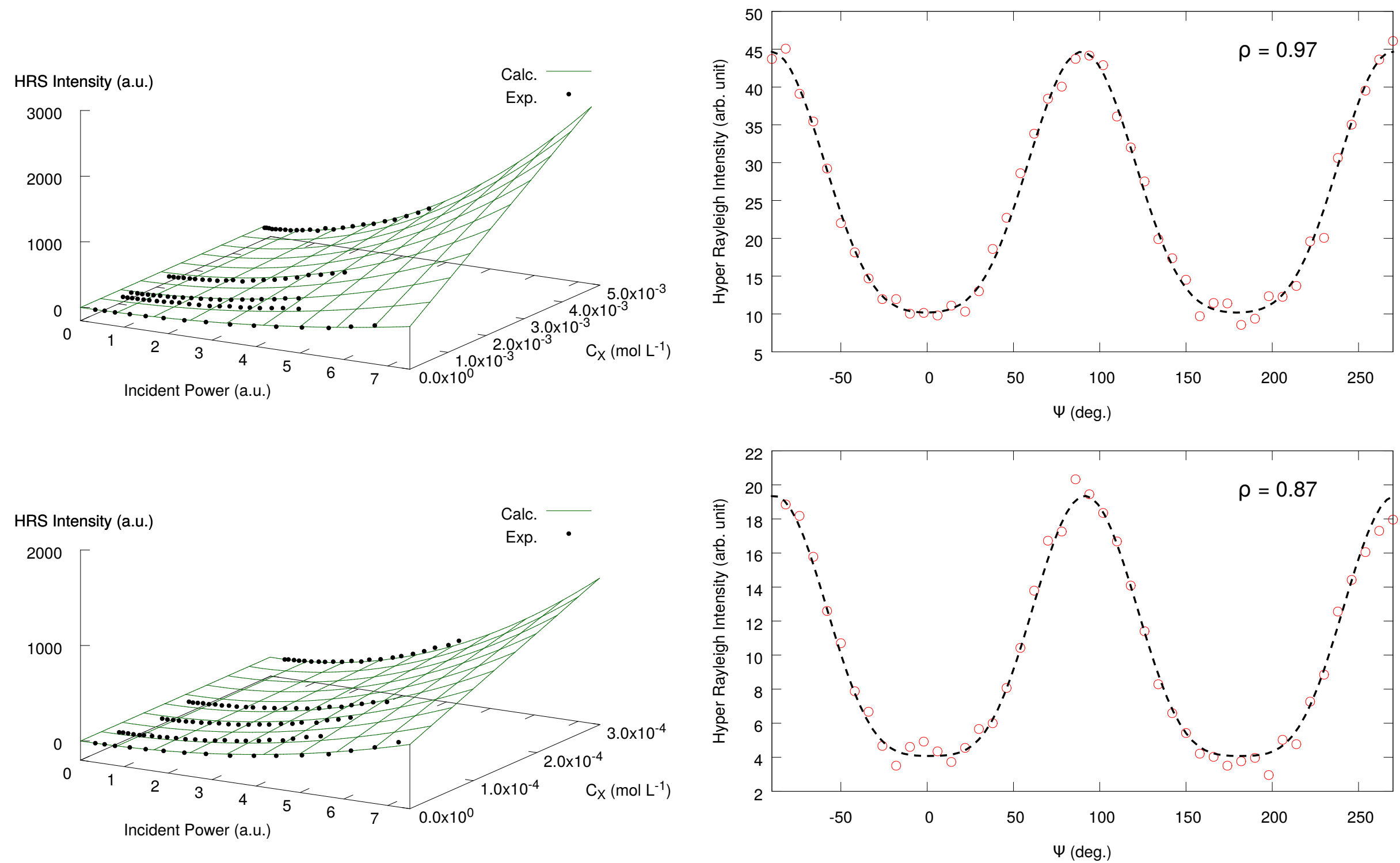

Figure S2. Power scan (left) and polarization scan (right) of closed (top) and protonated open (bottom) forms of 3. 

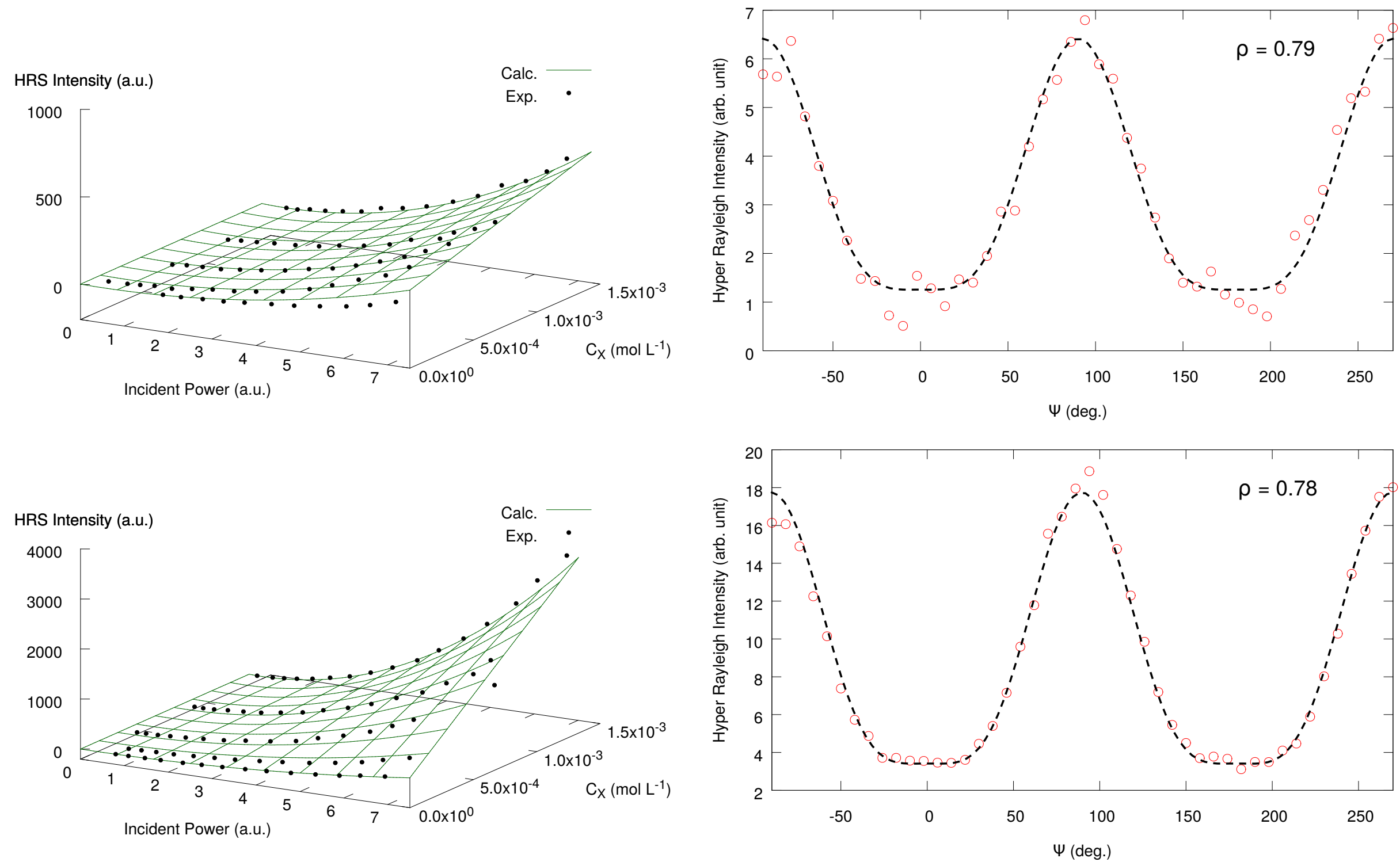

Figure S3. Power scan (left) and polarization scan (right) of closed (top) and protonated open (bottom) forms of 4. 

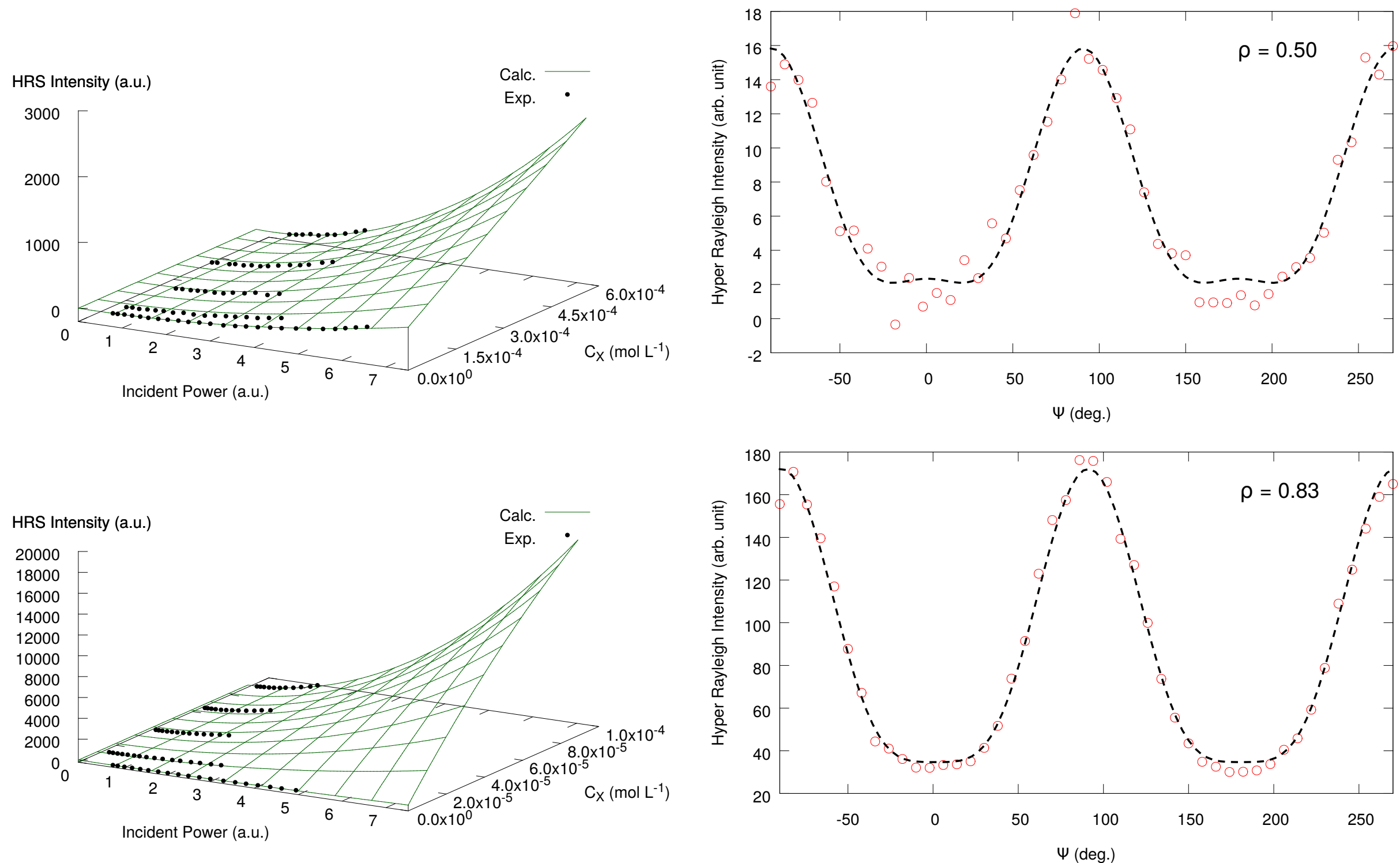

Figure S4. Power scan (left) and polarization scan (right) of closed (top) and protonated open (bottom) forms of 11. 

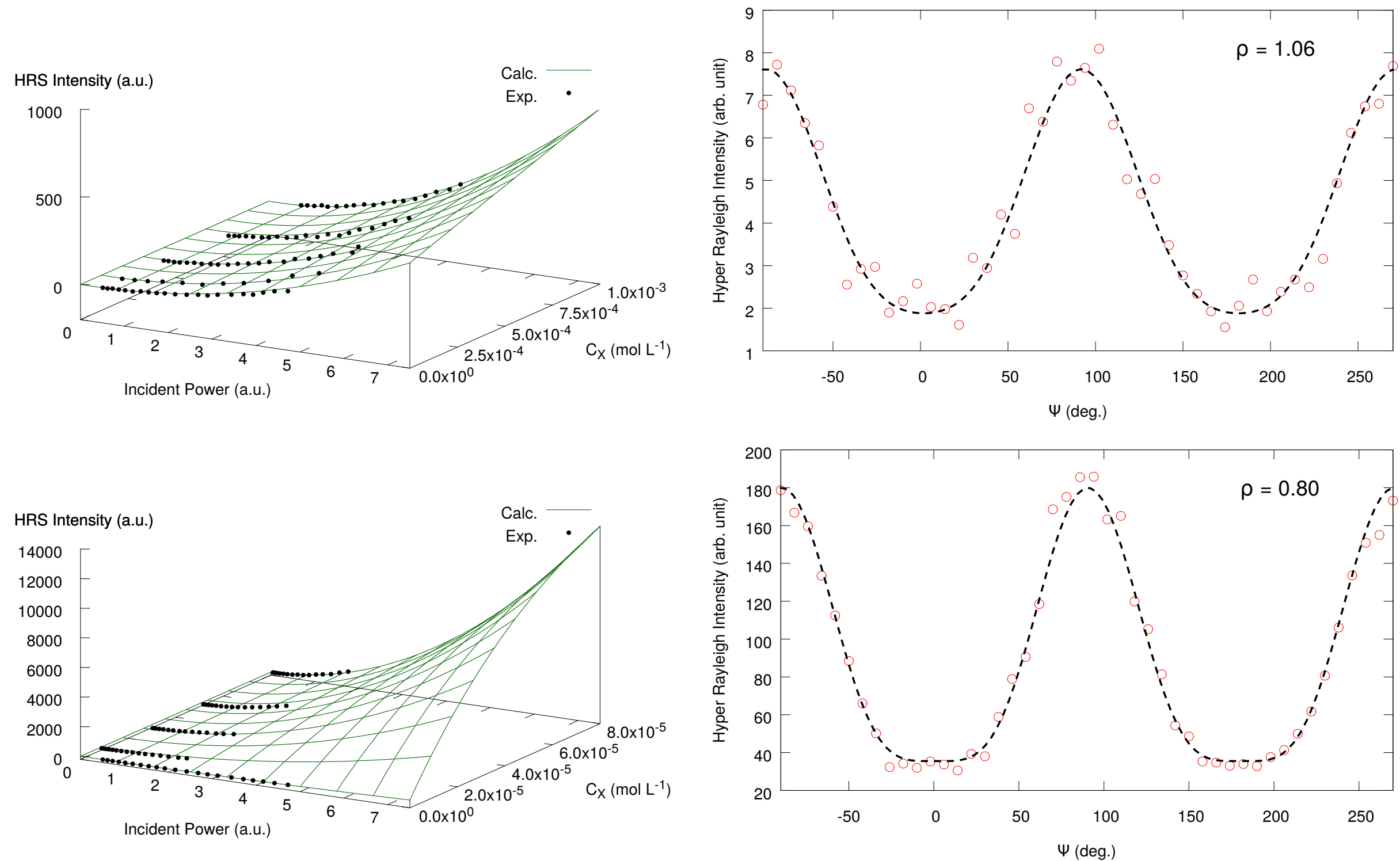

Figure S5. Power scan (left) and polarization scan (right) of closed (top) and protonated open (bottom) forms of 12. 


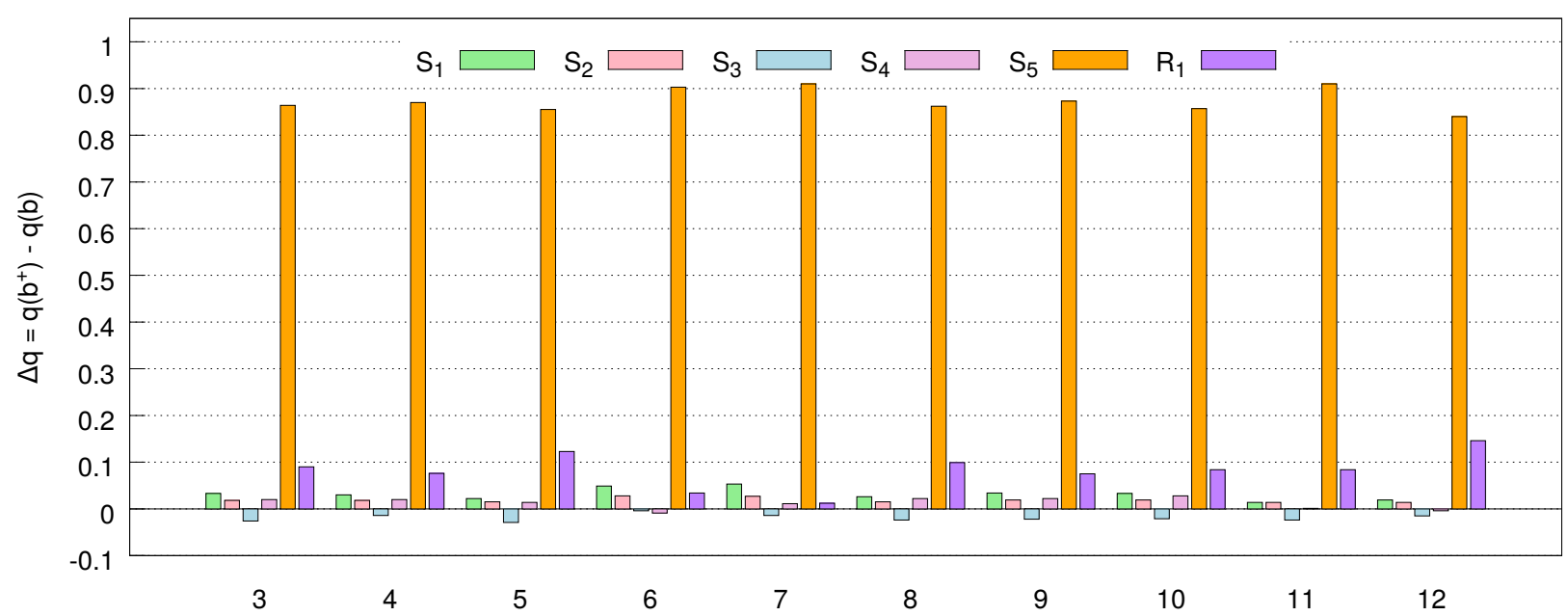

Figure S6a. Variations of the Mulliken charges from the zwitterionic to protonated open forms, $\Delta \mathrm{q}$ $=q\left(\mathbf{b}^{+}\right)-q(\mathbf{b})$ of the oxazines (3-12) as determined at the IEF-PCM/M06/6-311G(d) level (in chloroform). 

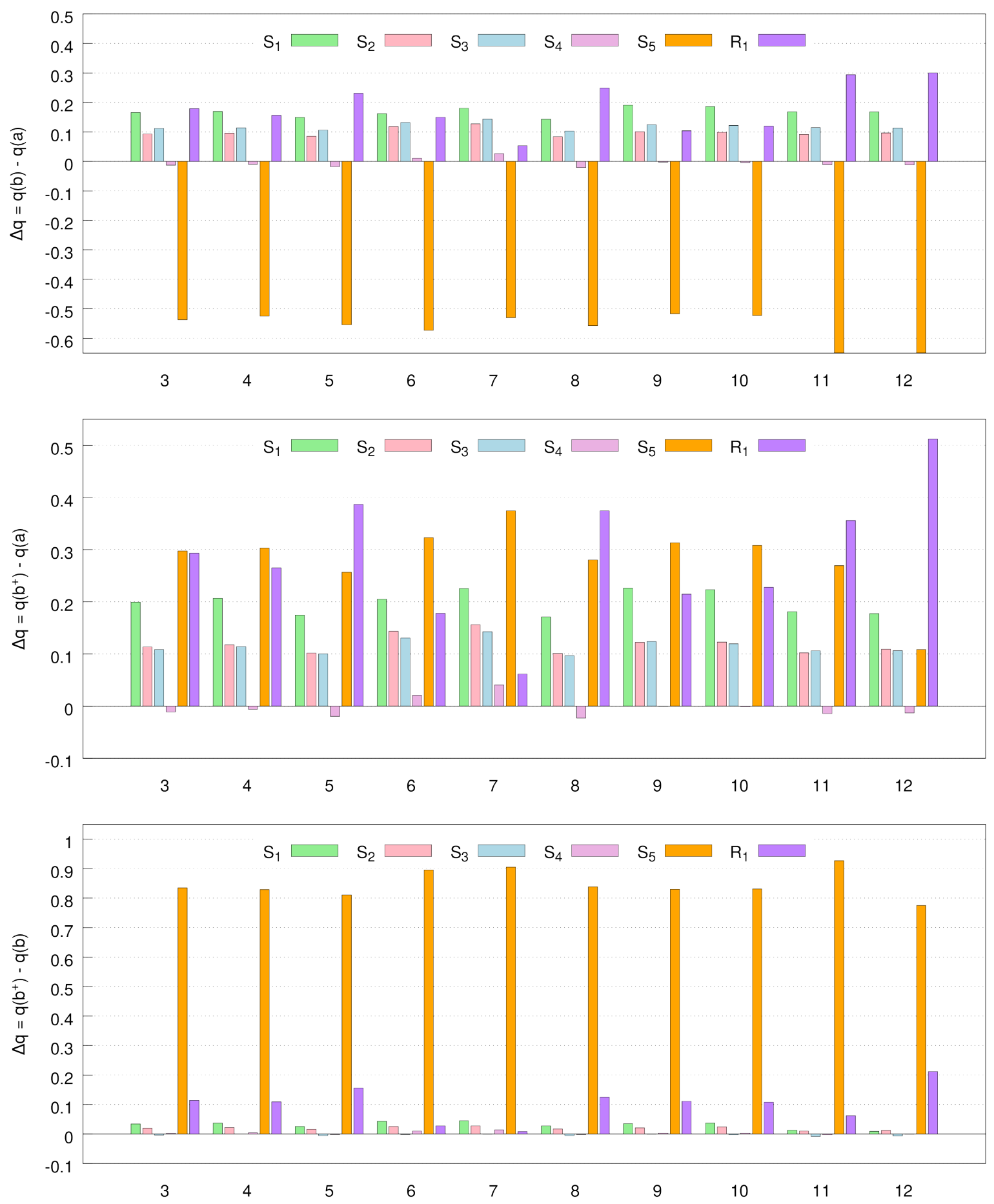

Figure S6b Spatial distribution on the $S_{1}-S_{5}$ and $R_{1}$ molecular moieties of the variations of the Hirshfeld charges when opening the oxazine, as determined at the IEF-PCM/M06/6-311G(d) level (in chloroform). Top) photo-triggered opening, $\Delta q=q(b)-q(a)$; middle) acido-triggered opening, $\Delta q=q\left(\mathbf{b}^{+}\right)-q(\mathbf{a})$ and bottom) from the photo- to the acido-triggered open forms, $\Delta q=q\left(\mathbf{b}^{+}\right)-q(\mathbf{b})$. 

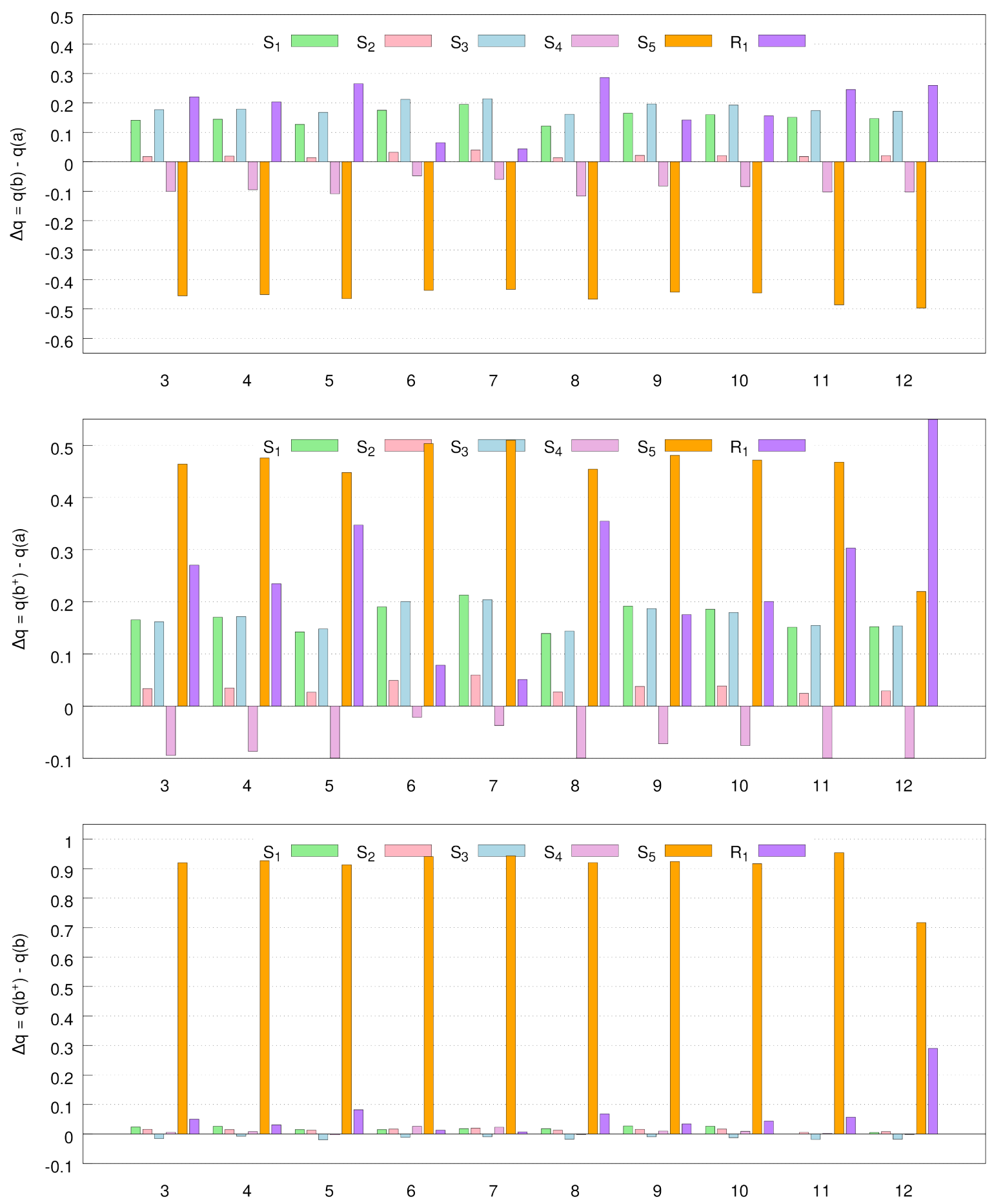

Figure S6c Spatial distribution on the $S_{1}-S_{5}$ and $R_{1}$ molecular moieties of the variations of the NPA (natural population analysis) charges when opening the oxazine, as determined at the IEFPCM/M06/6-311G(d) level (in chloroform). Top) photo-triggered opening, $\Delta q=q(\mathbf{b})-q(\mathbf{a})$; middle) acido-triggered opening, $\Delta q=q\left(b^{+}\right)-q(a)$ and bottom) from the photo- to the acido-triggered open forms, $\Delta q=q\left(b^{+}\right)-q(b)$. 
Figure S7(1-12). IEF-PCM(solvent = chloroform)/TDDFT/M06-2X/6-311+G(d) UV/vis absorption spectra of the closed (a), photochemically open (b), and acidly open $(\mathbf{b}+)$ forms of compounds 1-12 together with details (excitation energies and wavelengths, oscillator strengths, and molecular orbitals involved in the major singly-excited determinants) on dominant low-energy transitions.

\section{Molecule 1}

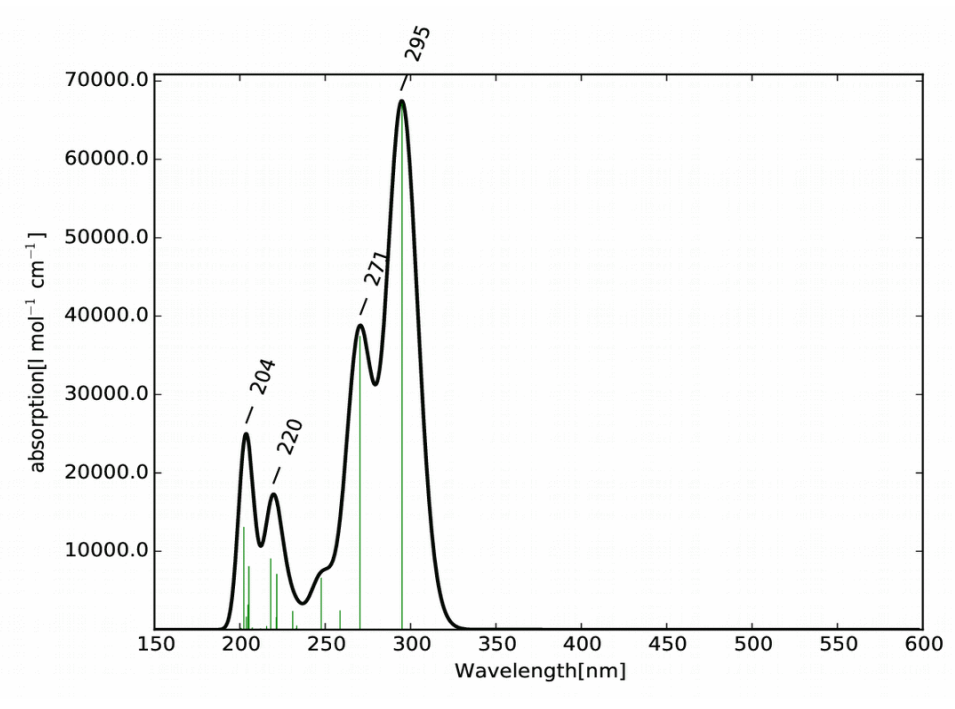

Closed (a)

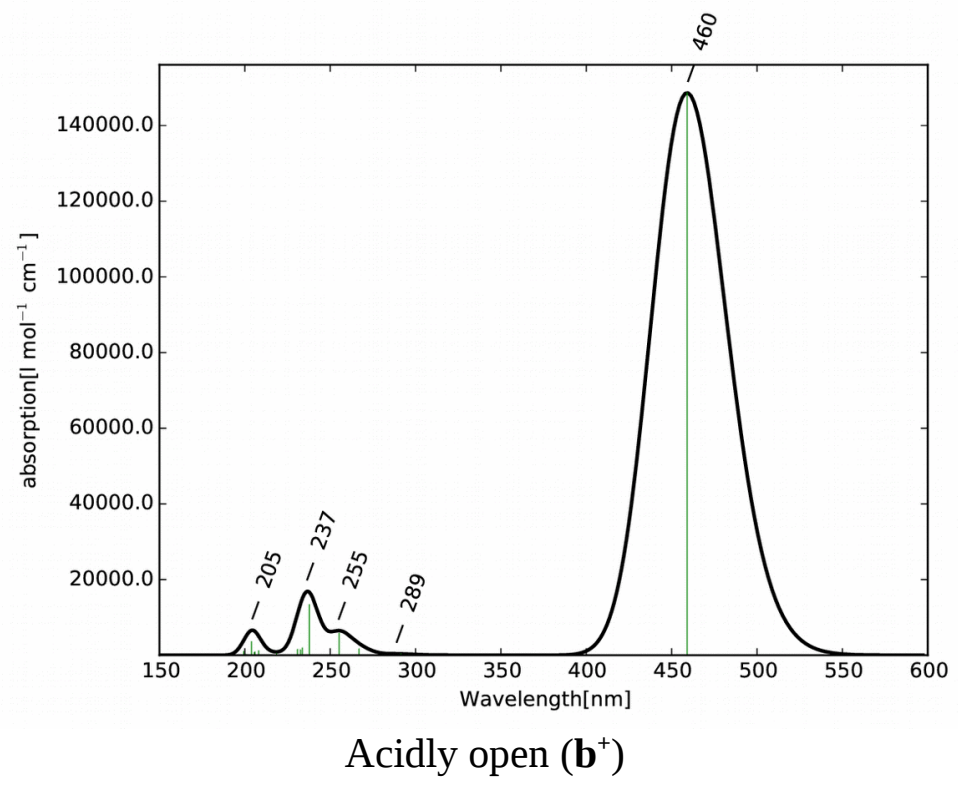

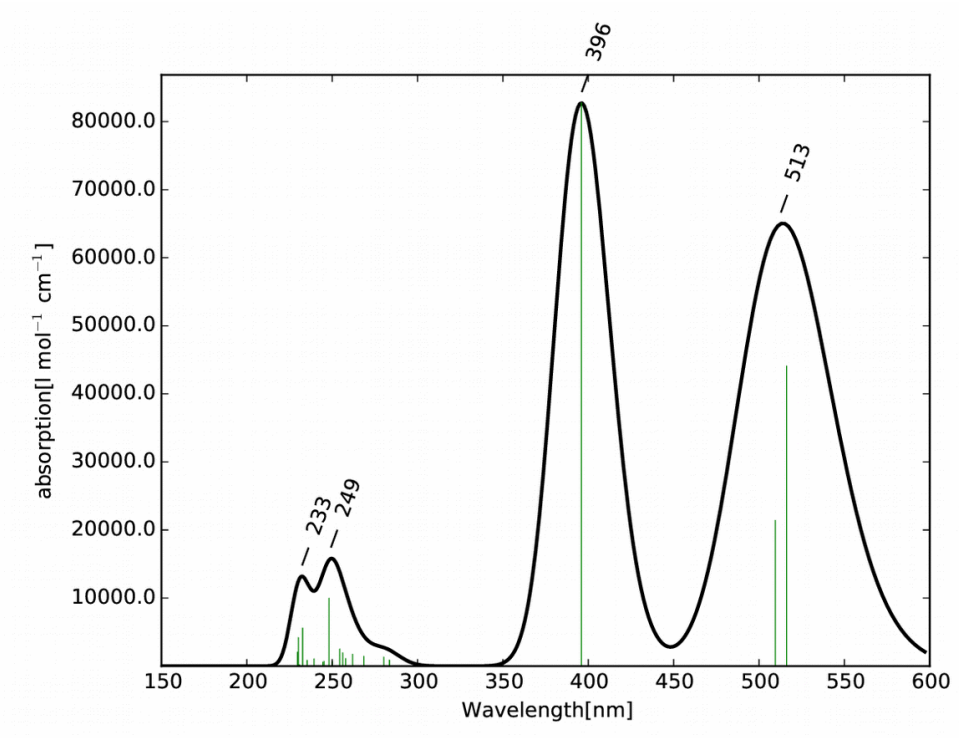

Photochemically open (b)

\begin{tabular}{cccccc}
\hline Root & Energy $(\mathrm{eV})$ & Wavelength $(\mathrm{nm})$ & $\mathrm{f}$ & Transition & $\%$ \\
\hline \multicolumn{6}{c}{ Closed (a) } \\
1 & 4.203 & 295 & 0.746 & HOMO $\rightarrow$ LUMO & 89.6 \\
2 & 4.586 & 270 & 0.416 & HOMO $\rightarrow$ LUMO+5 & 69.8 \\
\hline \multicolumn{5}{c}{ Photochemically open $(\mathbf{b})$} \\
1 & 2.402 & 516 & 0.490 & HOMO $\rightarrow$ LUMO & 56.9 \\
2 & 2.433 & 510 & 0.238 & HOMO- $\rightarrow$ LUMO & 57.3 \\
3 & 3.311 & 395 & 0.921 & HOMO-2 $\rightarrow$ LUMO & 94.9 \\
\hline \multicolumn{7}{c}{ Acidly open $\left(\mathbf{b}^{+}\right)$} \\
1 & 2.701 & 459 & 1.654 & HOMO $\rightarrow$ LUMO & 96.6 \\
6 & 5.213 & 238 & 0.149 & HOMO $\rightarrow$ LUMO+1 & 31.9 \\
\hline
\end{tabular}




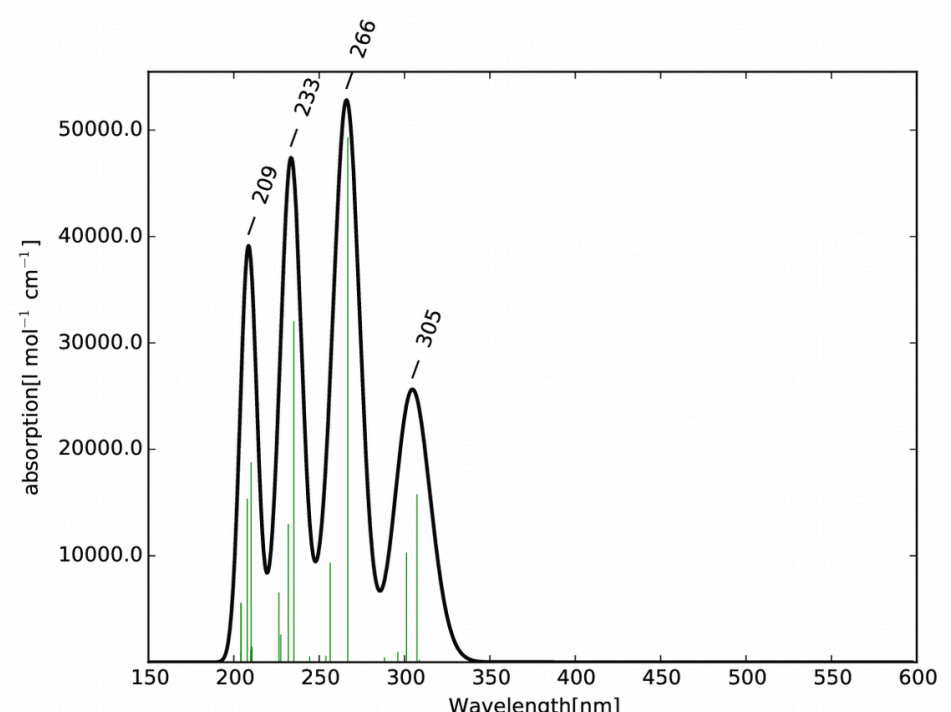

Closed (a)

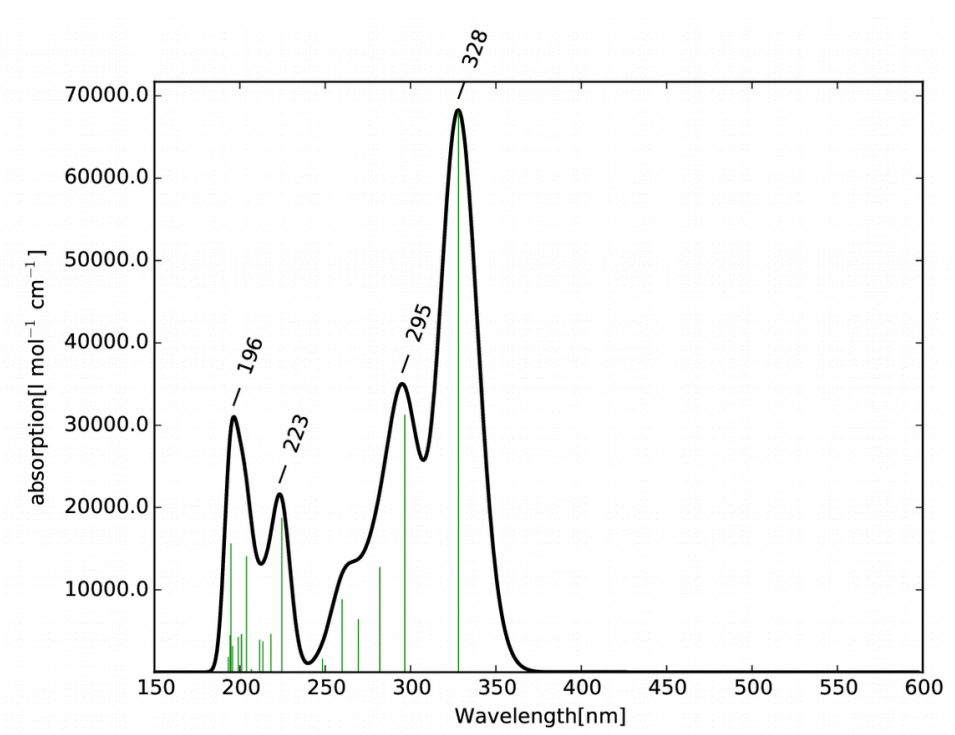

Acidly open ( $\left.\mathbf{b}^{+}\right)$

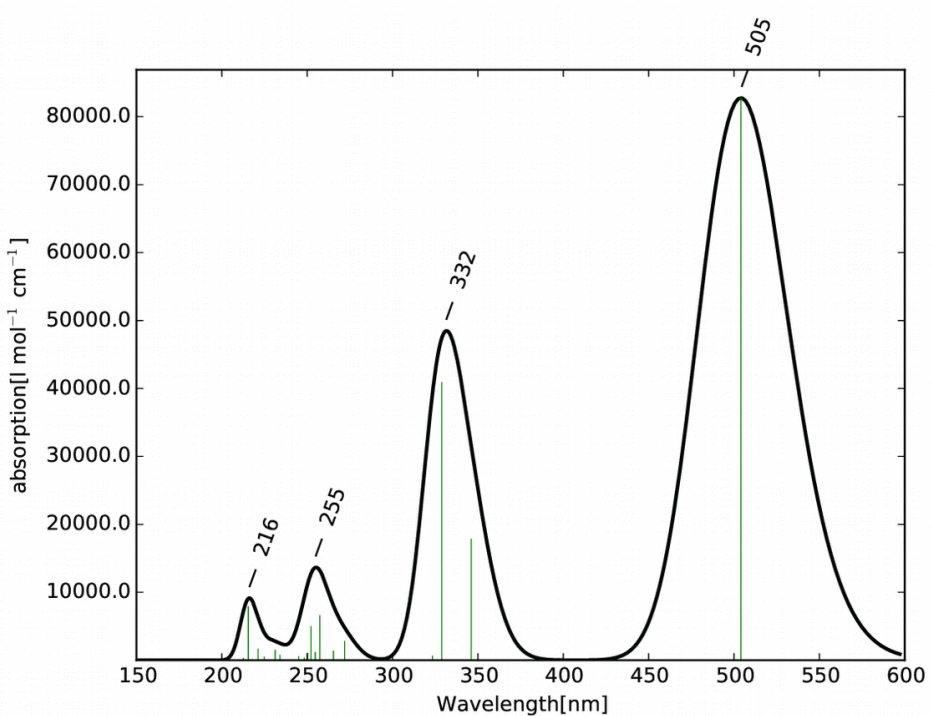

Photochemically open (b)

\begin{tabular}{|c|c|c|c|c|c|}
\hline Root & Energy $(\mathrm{eV})$ & Wavelength (nm) & $\mathrm{f}$ & Transition & $\%$ \\
\hline \multicolumn{6}{|c|}{ Closed (a) } \\
\hline 2 & 4.035 & 307 & 0.175 & $\left\{\begin{array}{l}\text { HOMO } \rightarrow \text { LUMO } \\
\text { HOMO- } 1 \rightarrow \text { LUMO }\end{array}\right.$ & $\begin{array}{l}37.5 \\
28.9\end{array}$ \\
\hline 3 & 4.117 & 301 & 0.114 & HOMO-1 $\rightarrow$ LUMO & 59.8 \\
\hline 7 & 4.647 & 267 & 0.548 & HOMO-1 $\rightarrow$ LUMO+1 & 83.2 \\
\hline 11 & 5.273 & 235 & 0.356 & $\left\{\begin{array}{l}\text { HOMO-2 } \rightarrow \text { LUMO+1 } \\
\text { HOMO-3 } \rightarrow \text { LUMO+1 }\end{array}\right.$ & $\begin{array}{l}43.8 \\
36.0\end{array}$ \\
\hline 16 & 5.900 & 210 & 0.209 & HOMO-2 $\rightarrow$ LUMO+3 & 22.9 \\
\hline \multicolumn{6}{|c|}{ Photochemically open (b) } \\
\hline 1 & 2.460 & 504 & 0.921 & HOMO $\rightarrow$ LUMO & 96.6 \\
\hline 3 & 3771 & 328 & 0.455 & HOMO-1 $\rightarrow$ LUMO & 83.3 \\
\hline \multicolumn{6}{|c|}{ Acidly open $\left(\mathbf{b}^{+}\right)$} \\
\hline 1 & 3.780 & 328 & 0.757 & HOMO $\rightarrow$ LUMO & 66.9 \\
\hline 3 & 4.180 & 297 & 0.347 & HOMO-1 $\rightarrow$ LUMO & 51.5 \\
\hline 9 & 5.521 & 225 & 0.208 & $\mathrm{HOMO} \rightarrow \mathrm{LUMO}+2$ & 57.2 \\
\hline
\end{tabular}




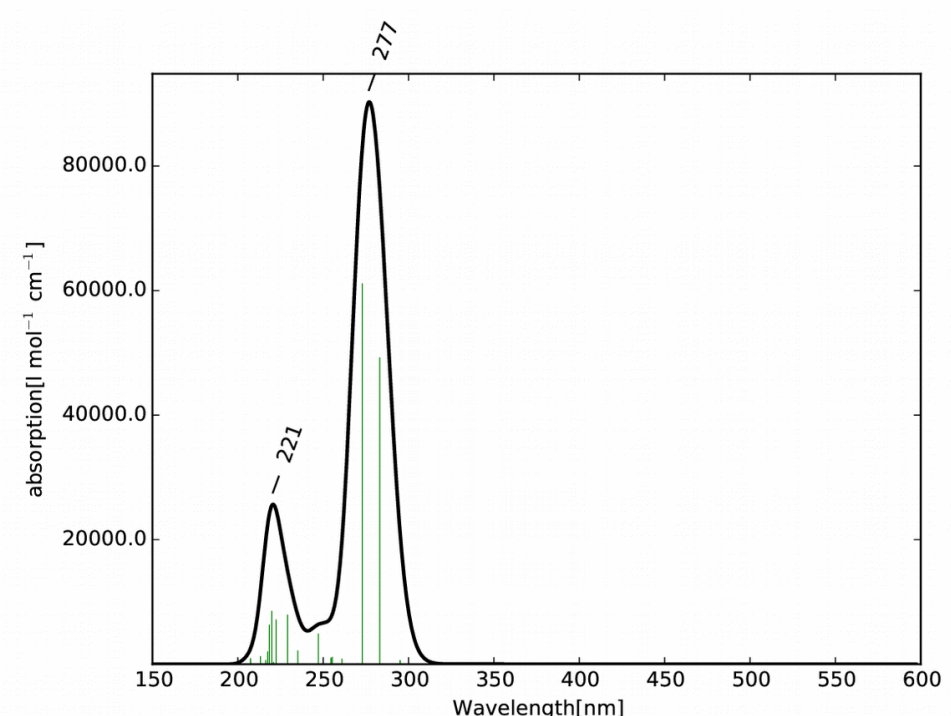

Closed (a)

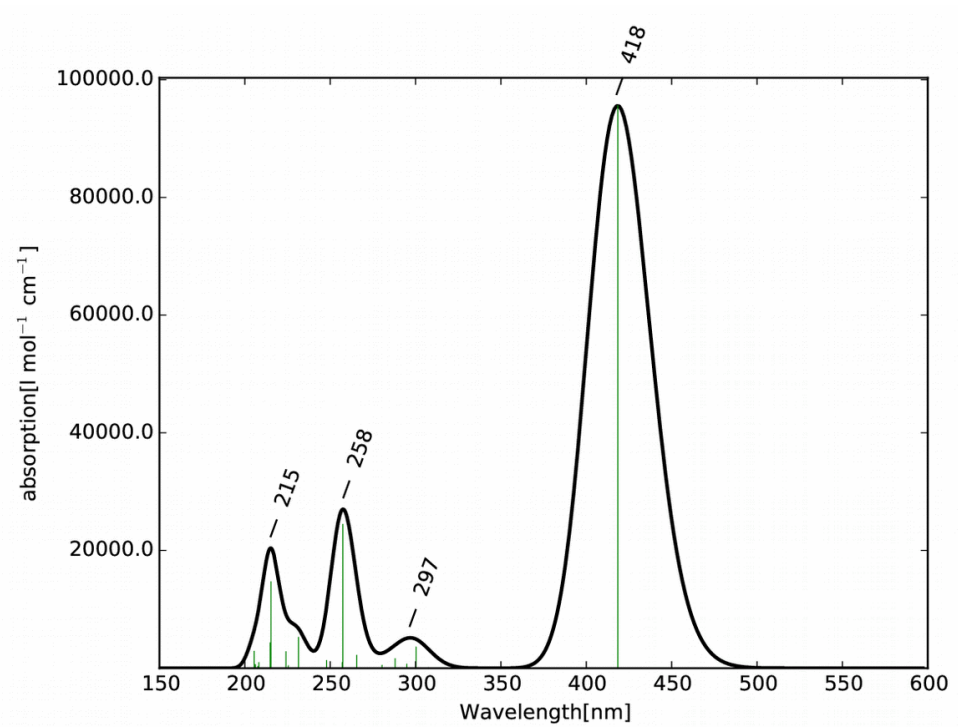

Acidly open ( $\mathbf{b}^{+}$)

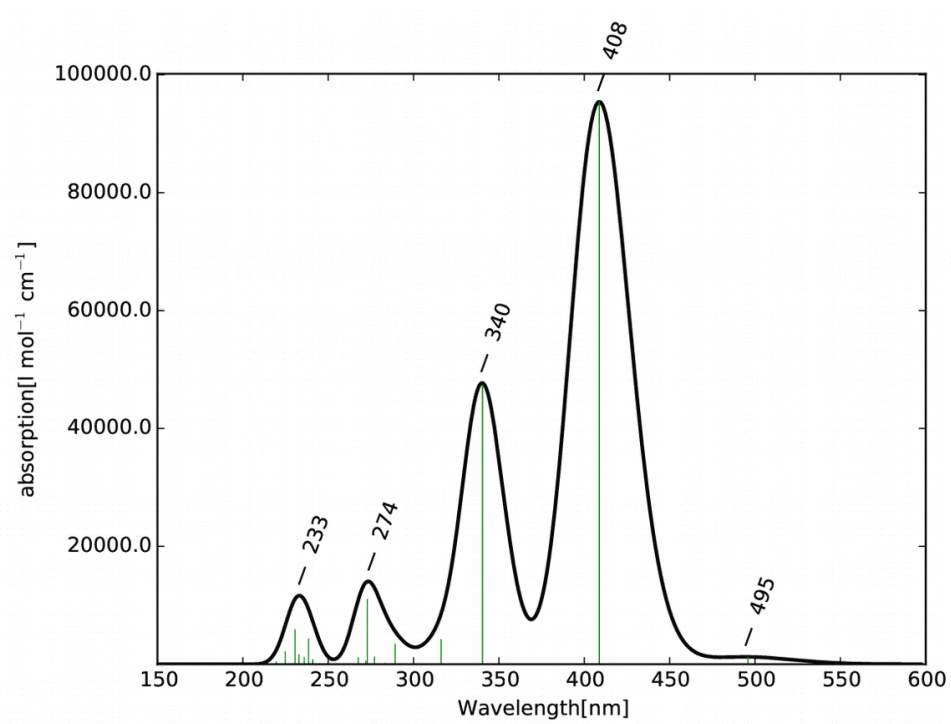

Photochemically open (b)

\begin{tabular}{cccccc}
\hline Root & Energy $(\mathrm{eV})$ & Wavelength $(\mathrm{nm})$ & $\mathrm{f}$ & Transition & $\%$ \\
\hline \multicolumn{7}{c}{ Closed $(\mathbf{a})$} \\
3 & 4.379 & 283 & 0.548 & HOMO-2 $\rightarrow$ LUMO & 58.3 \\
4 & 4.543 & 273 & 0.680 & HOMO- $1 \rightarrow$ LUMO+1 & 66.7 \\
\hline \multicolumn{7}{c}{ Photochemically open $(\mathbf{b})$} \\
3 & 3.033 & 409 & 1.062 & HOMO-1 $\rightarrow$ LUMO & 96.9 \\
3 & 3.643 & 340 & 0.527 & HOMO $\rightarrow$ LUMO+1 & 92.7 \\
\hline & 2.963 & Acidly open $\left(\mathbf{b}^{+}\right)$ & \\
9 & 4.817 & 418 & 1.064 & HOMO $\rightarrow$ LUIMO & 97.0 \\
\hline
\end{tabular}




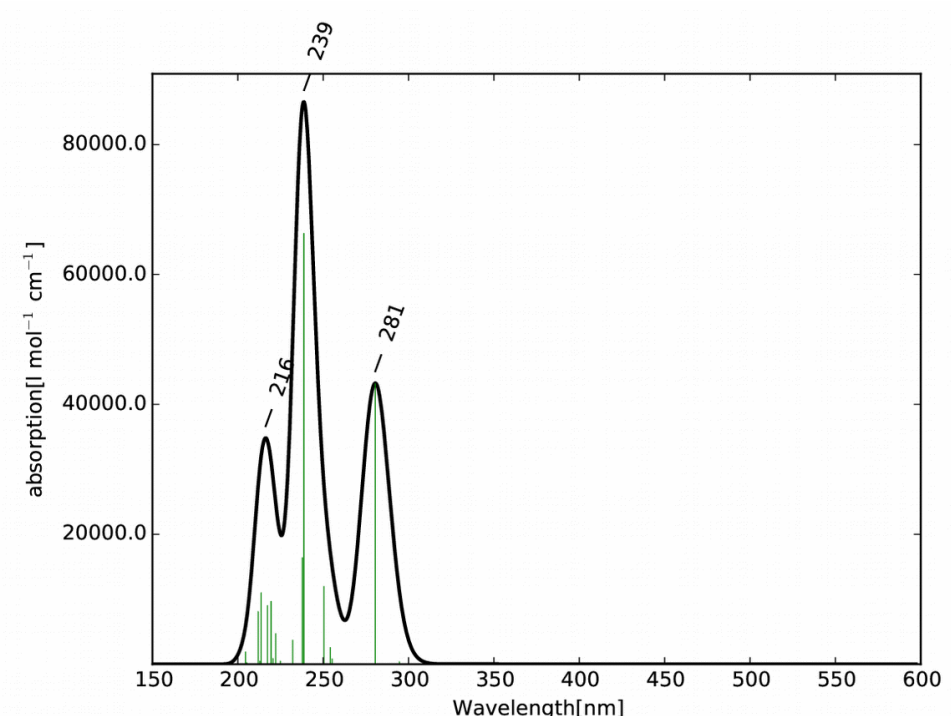

Closed (a)

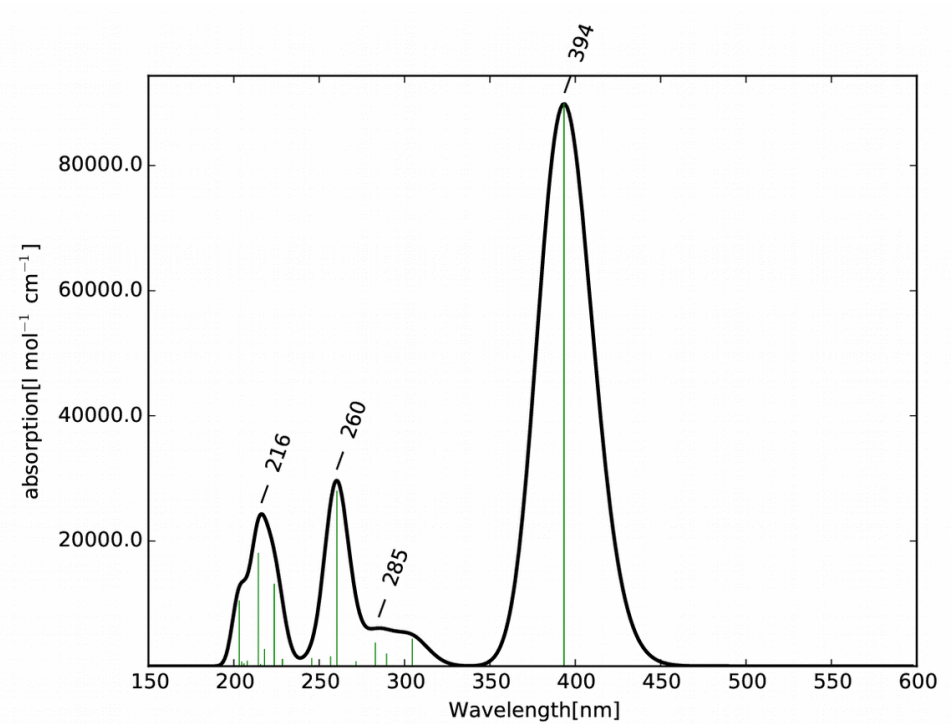

Acidly open $\left(\mathbf{b}^{+}\right)$

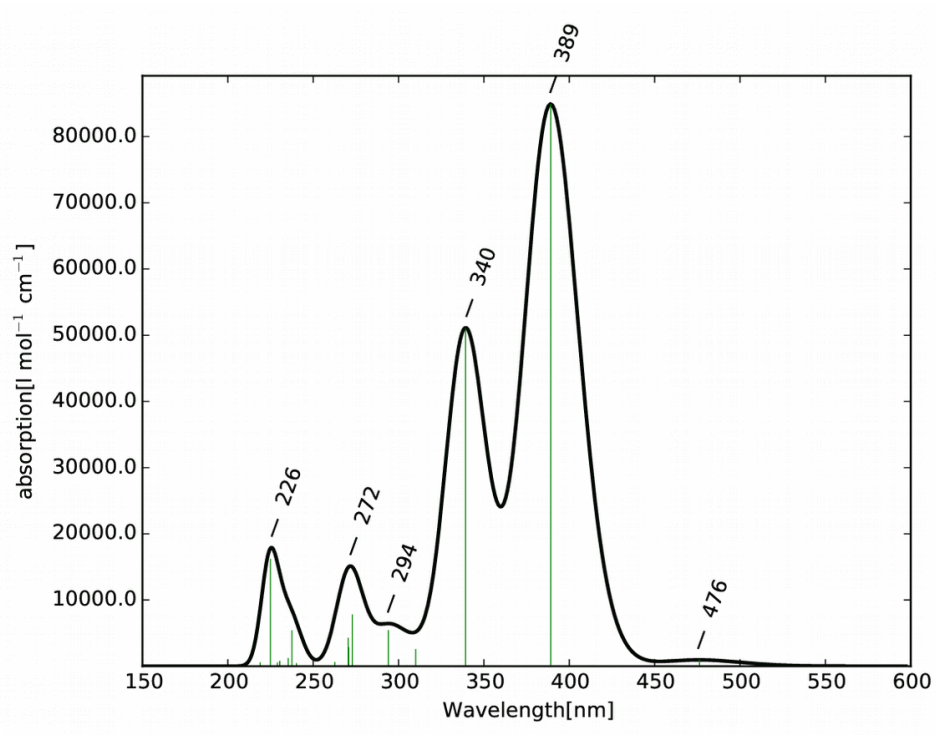

Photochemically open (b)

\begin{tabular}{|c|c|c|c|c|c|}
\hline Root & Energy $(\mathrm{eV})$ & Wavelength (nm) & $\mathrm{f}$ & Transition & $\%$ \\
\hline \multicolumn{6}{|c|}{ Closed (a) } \\
\hline 3 & 4.420 & 280 & 0.480 & HOMO-2 $\rightarrow$ LUMO & 80.3 \\
\hline 8 & 5.194 & 238 & 0.738 & HOMO-1 $\rightarrow$ LUMO+1 & 67.2 \\
\hline \multicolumn{6}{|c|}{ Photochemically open (b) } \\
\hline 2 & 3.186 & 389 & 0.944 & HOMO-1 $\rightarrow$ LUMO & 96.9 \\
\hline 3 & 3.654 & 339 & 0.567 & HOMO $\rightarrow$ LUMO+1 & 94.2 \\
\hline \multicolumn{6}{|c|}{ Acidly open $\left(\mathbf{b}^{+}\right)$} \\
\hline 1 & 3.151 & 393 & 1.000 & HOMO $\rightarrow$ LUMO & 97.1 \\
\hline 8 & 4.761 & 260 & 0.311 & HOMO-1 $\rightarrow$ LUMO+1 & 79.7 \\
\hline 16 & 5.783 & 214 & 0.201 & HOMO-1 $\rightarrow$ LUMO+2 & 41.9 \\
\hline
\end{tabular}




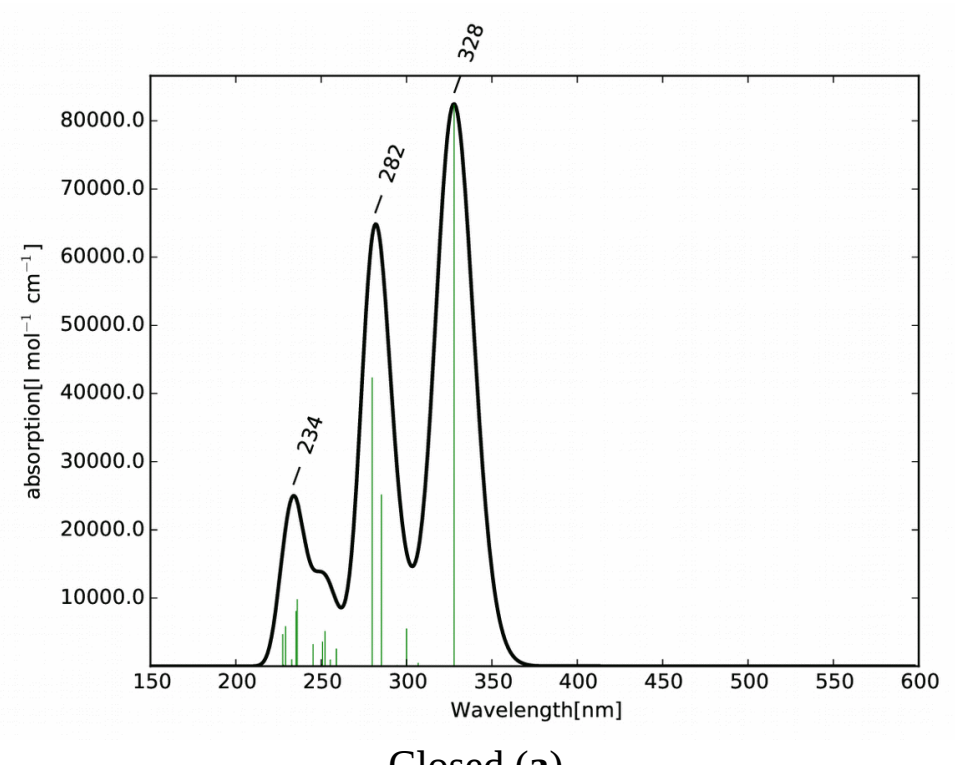

Closed (a)

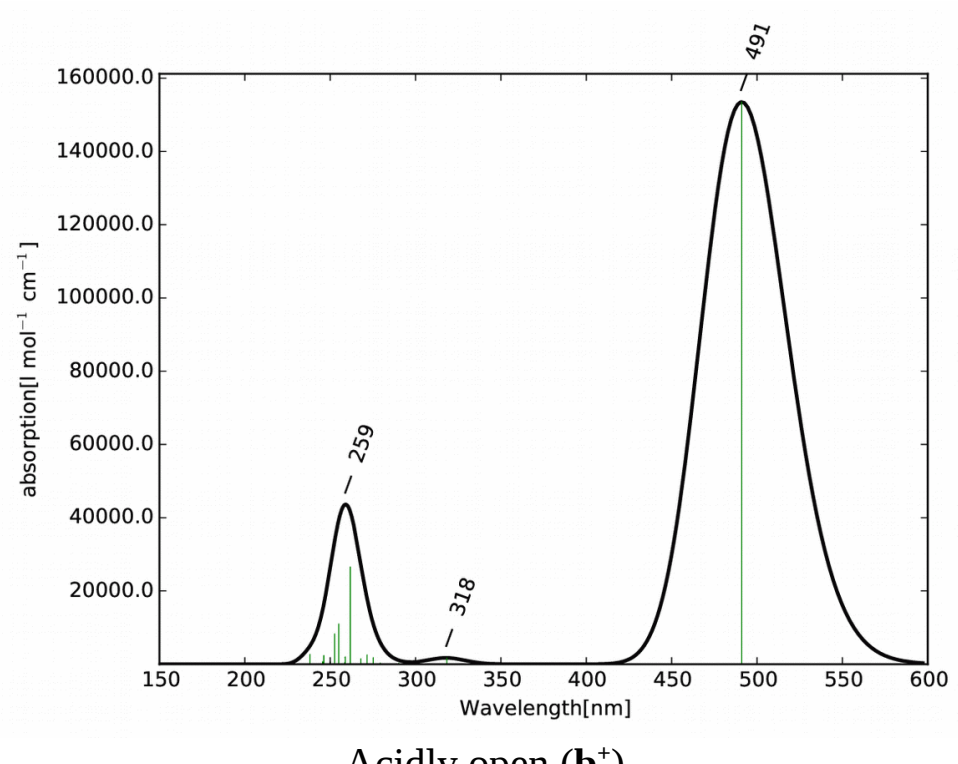

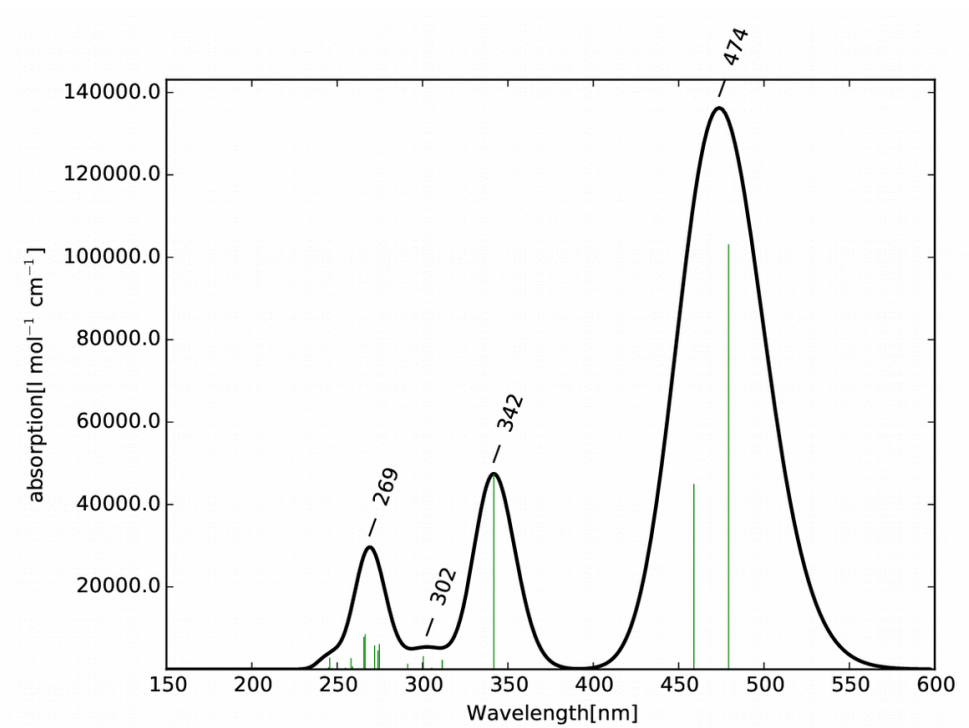

Photochemically open (b)

\begin{tabular}{|c|c|c|c|c|c|}
\hline Root & Energy $(\mathrm{eV})$ & Wavelength (nm) & $\mathrm{f}$ & Transition & $\%$ \\
\hline \multicolumn{6}{|c|}{ Closed (a) } \\
\hline 1 & 3.782 & 328 & 0.916 & $\mathrm{HOMO} \rightarrow \mathrm{LUMO}+1$ & 90.1 \\
\hline 6 & 4.347 & 285 & 0.280 & $\mathrm{HOMO} \rightarrow \mathrm{LUMO}+4$ & 57.5 \\
\hline 7 & 4.430 & 280 & 0.470 & HOMO-3 $\rightarrow$ LUMO & 71.8 \\
\hline \multicolumn{6}{|c|}{ Photochemically open (b) } \\
\hline 1 & 2.587 & 479 & 1.147 & HOMO $\rightarrow$ LUMO & 62.8 \\
\hline 2 & 2.701 & 458 & 0.500 & HOMO-1 $\rightarrow$ LUMO & 61.8 \\
\hline 3 & 3.628 & 341 & 0.528 & HOMO $\rightarrow$ LUMO+1 & 85.0 \\
\hline \multicolumn{6}{|c|}{ Acidly open $\left(\mathbf{b}^{+}\right)$} \\
\hline 1 & 2.525 & 491 & 1.708 & HOMO $\rightarrow$ LUMO & 93.6 \\
\hline 11 & 4.736 & 262 & 0.295 & HOMO-4 $\rightarrow$ LUMO+1 & 88.5 \\
\hline
\end{tabular}




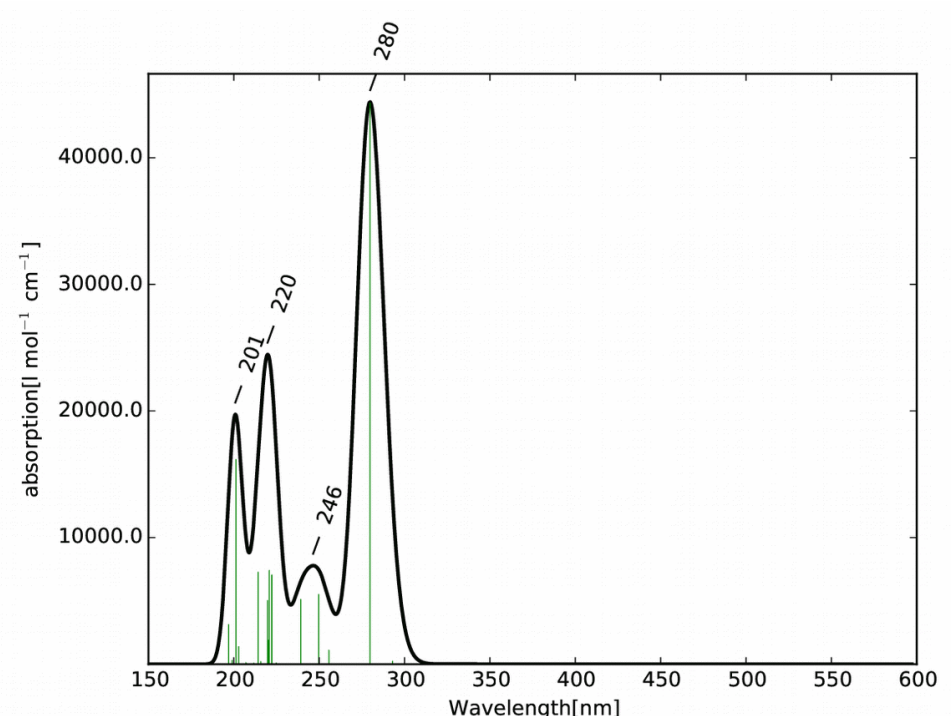

Closed (a)

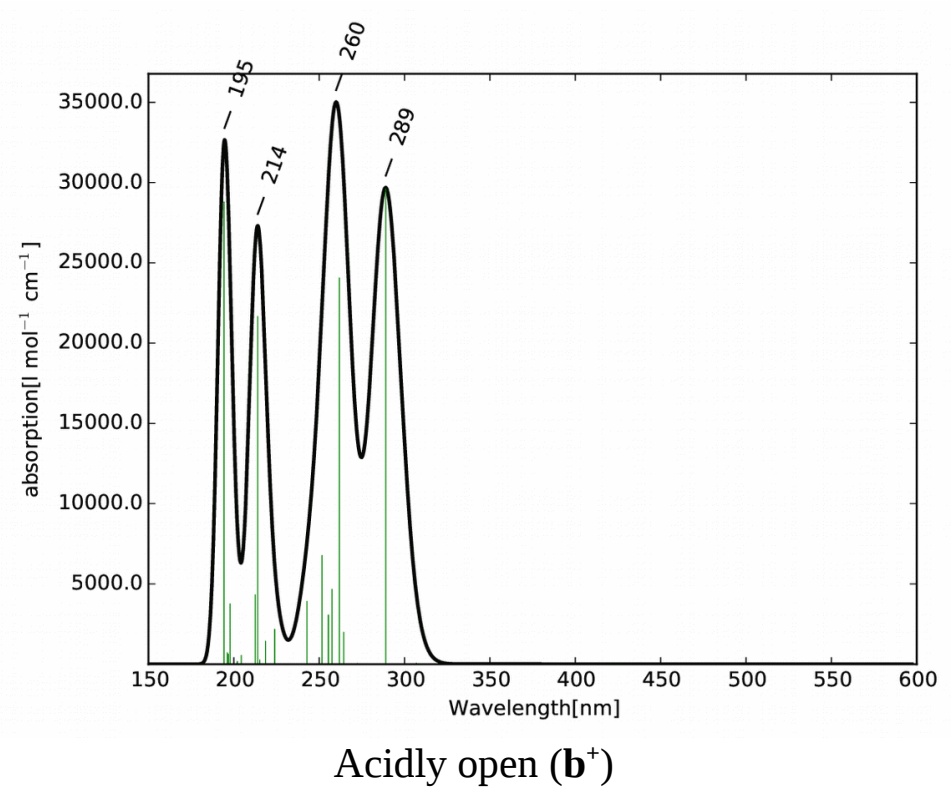

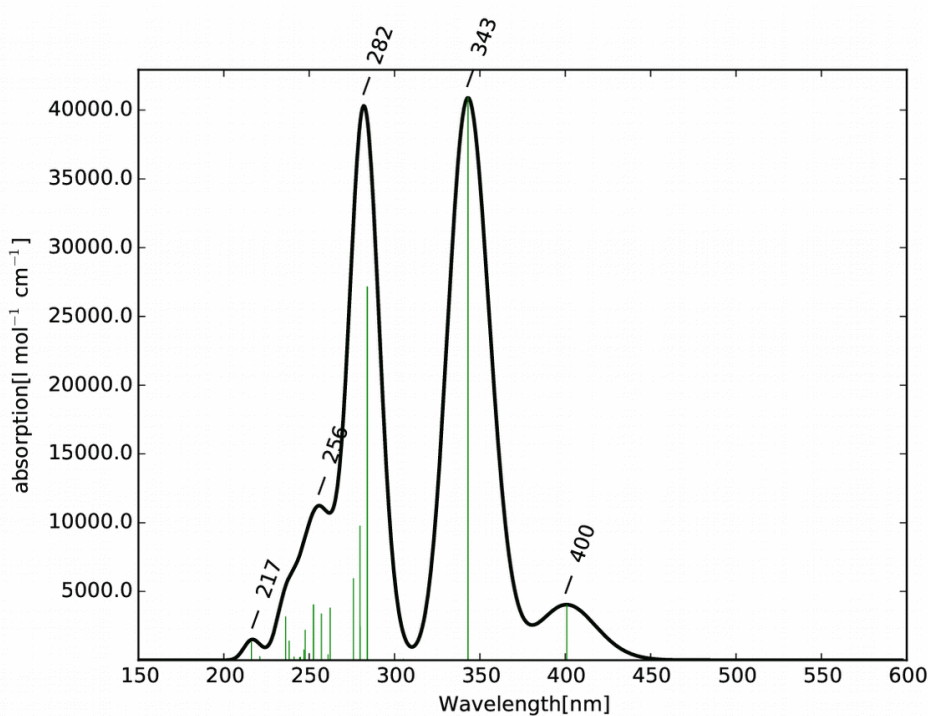

Photochemically open (b)

\begin{tabular}{|c|c|c|c|c|c|}
\hline Root & Energy $(\mathrm{eV})$ & Wavelength (nm) & $\mathrm{f}$ & Transition & $\%$ \\
\hline \multicolumn{6}{|c|}{ Closed (a) } \\
\hline 3 & 4.432 & 280 & 0.493 & HOMO-1 $\rightarrow$ LUMO & 82.7 \\
\hline 18 & 6.162 & 201 & 0.180 & $\begin{array}{l}\text { HOMO-3 } \rightarrow \text { LUMO+1 } \\
\text { HOMO-3 } \rightarrow \text { LUMO+3 }\end{array}$ & $\begin{array}{l}31.5 \\
22.0\end{array}$ \\
\hline \multicolumn{6}{|c|}{ Photochemically open (b) } \\
\hline 2 & 3.614 & 343 & 0.455 & $\mathrm{HOMO} \rightarrow \mathrm{LUMO}+1$ & 95.6 \\
\hline 4 & 4.365 & 284 & 0.302 & $\left\{\begin{array}{l}\text { HOMO-3 } \rightarrow \text { LUMO } \\
\text { HOMO-1 } \rightarrow \text { LUMO }\end{array}\right.$ & $\begin{array}{l}38.5 \\
30.9\end{array}$ \\
\hline \multicolumn{6}{|c|}{ Acidly open $\left(\mathbf{b}^{+}\right)$} \\
\hline 2 & 4.290 & 289 & 0.329 & $\left\{\begin{array}{l}\text { HOMO-1 } \rightarrow \text { LUMO } \\
\text { HOMO } \rightarrow \text { LUMO }\end{array}\right.$ & $\begin{array}{l}50.1 \\
40.5\end{array}$ \\
\hline 5 & 4.735 & 262 & 0.268 & $\mathrm{HOMO} \rightarrow \mathrm{LUMO}+1$ & 61.6 \\
\hline 13 & 5.795 & 214 & 0.241 & $\mathrm{HOMO} \rightarrow \mathrm{LUMO}+2$ & 40.9 \\
\hline 20 & 6.383 & 194 & 0.320 & HOMO-6 $\rightarrow$ LUMO+1 & 74.3 \\
\hline
\end{tabular}




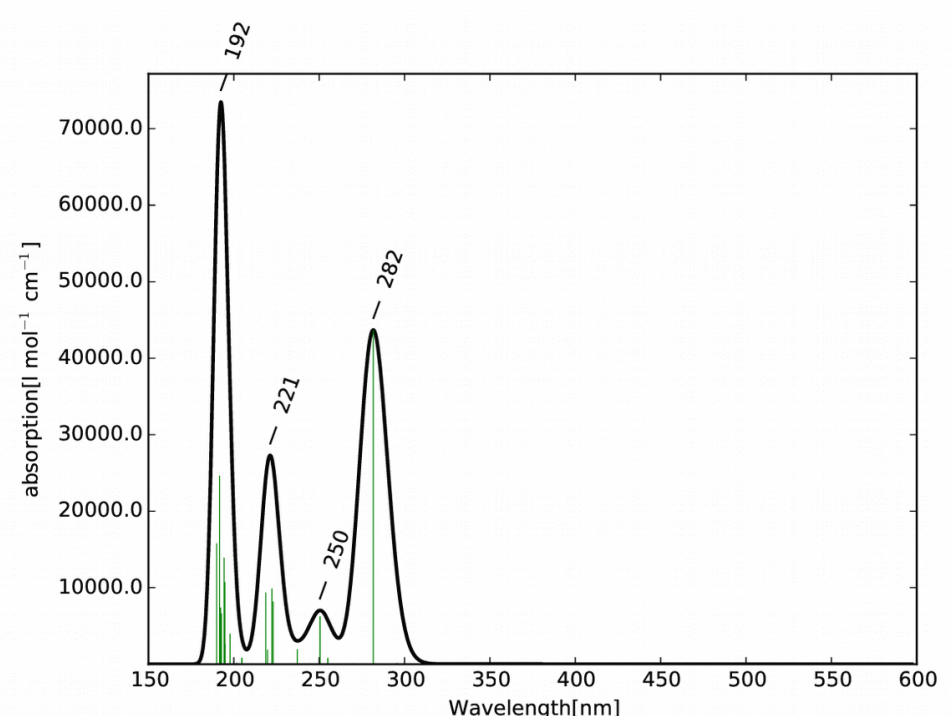

Closed (a)

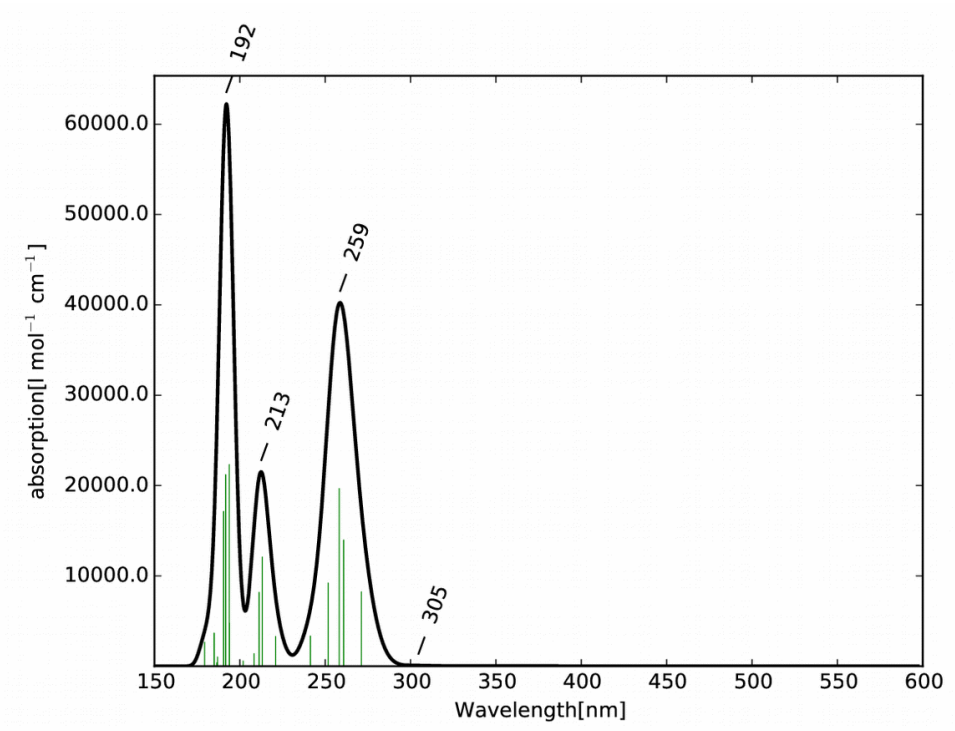

Acidly open $\left(\mathbf{b}^{+}\right)$

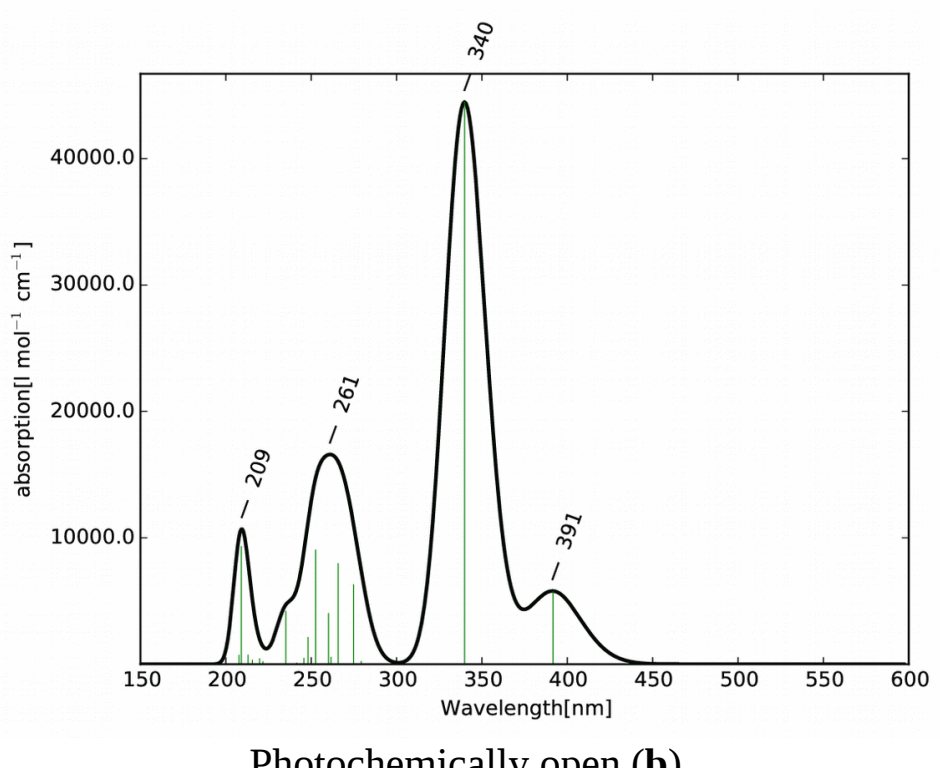

Photochemically open (b)

\begin{tabular}{|c|c|c|c|c|c|}
\hline Root & Energy $(\mathrm{eV})$ & Wavelength (nm) & $\mathrm{f}$ & Transition & $\%$ \\
\hline \multicolumn{6}{|c|}{ Closed (a) } \\
\hline 3 & 4.402 & 282 & 0.486 & HOMO-1 $\rightarrow$ LUMO & 93.8 \\
\hline 19 & 6.470 & 192 & 0.274 & $\left\{\begin{array}{l}\text { HOMO-3 } \rightarrow \text { LUMO+1 } \\
\text { HOMO-3 } \rightarrow \text { LUMO+3 }\end{array}\right.$ & $\begin{array}{c}36.4 \\
9.7\end{array}$ \\
\hline \multicolumn{6}{|c|}{ Photochemically open (b) } \\
\hline 2 & 3.648 & 340 & 0.495 & $\mathrm{HOMO} \rightarrow \mathrm{LUMO}+1$ & 96.0 \\
\hline \multicolumn{6}{|c|}{ Acidly open $\left(\mathbf{b}^{+}\right)$} \\
\hline 5 & 4.801 & 258 & 0.219 & $\mathrm{HOMO} \rightarrow \mathrm{LUMO}+1$ & 39.1 \\
\hline 14 & 6.369 & 194 & 0.248 & HOMO-4 $\rightarrow$ LUMO+1 & 67.1 \\
\hline 15 & 6.468 & 192 & 0.236 & HOMO-2 $\rightarrow$ LUMO+3 & 37.4 \\
\hline
\end{tabular}




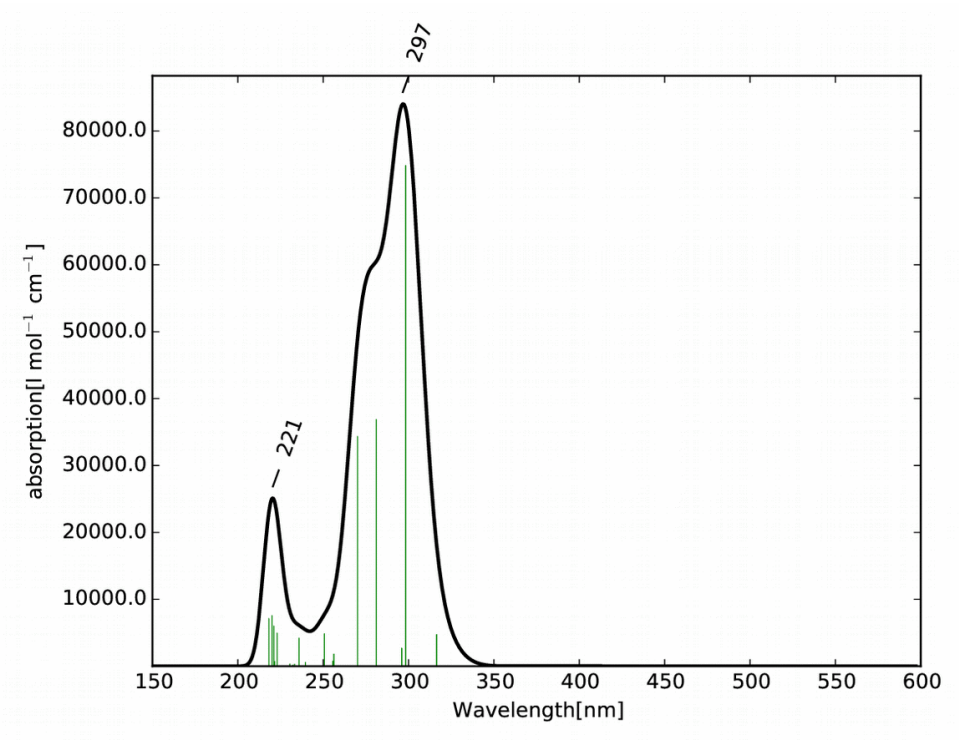

Closed (a)

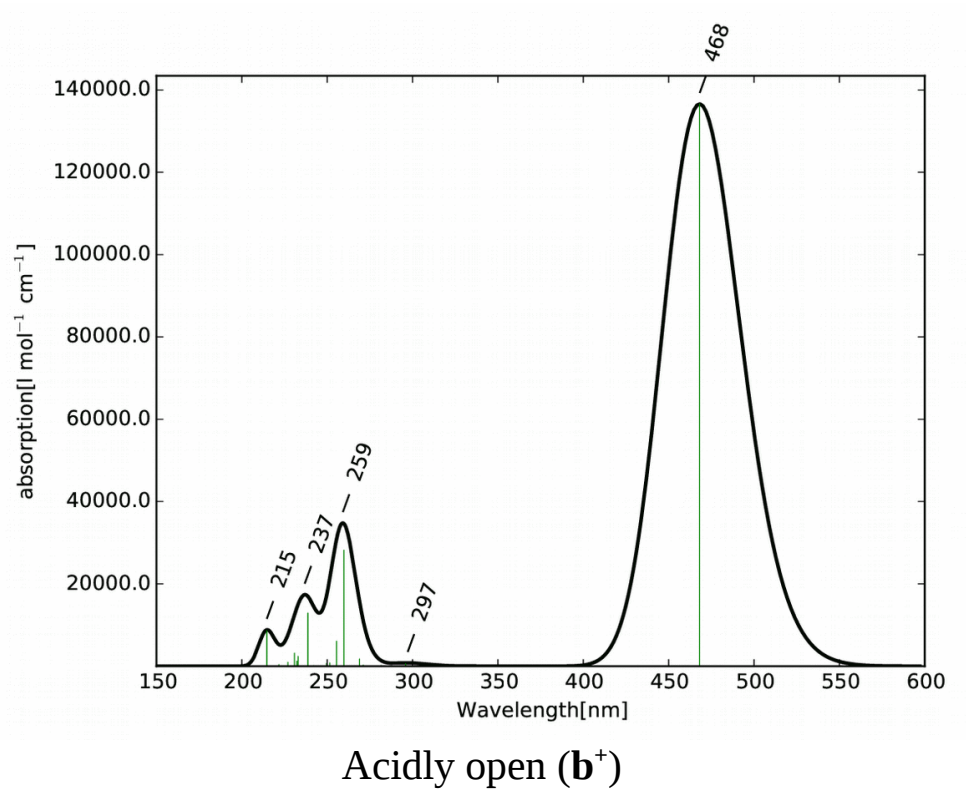

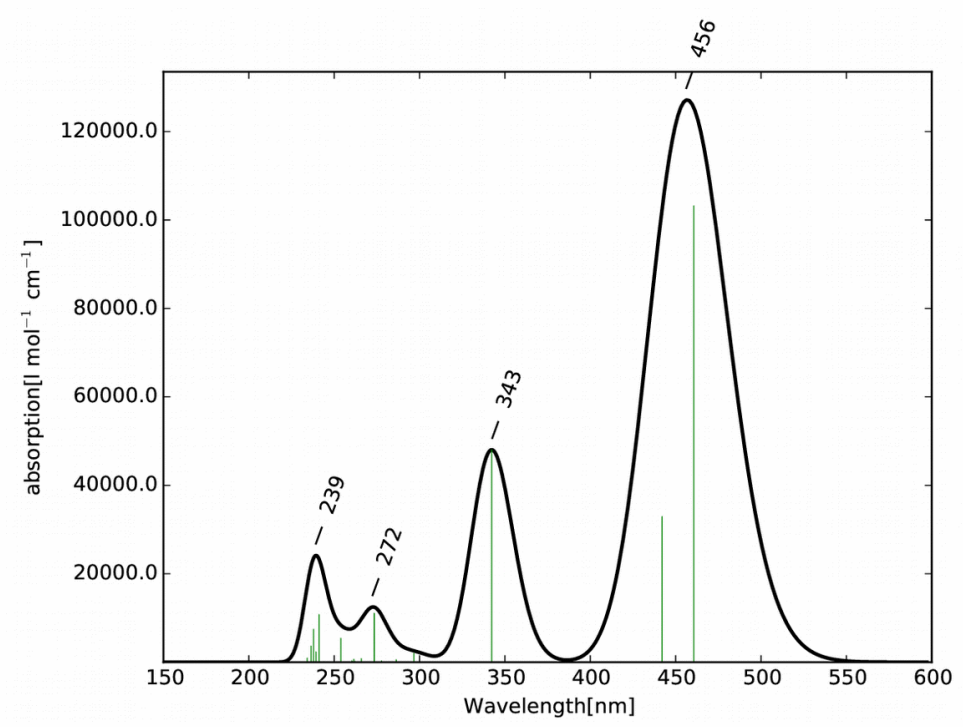

Photochemically open (b)

\begin{tabular}{|c|c|c|c|c|c|}
\hline Root & Energy (eV) & Wavelength (nm) & $\mathrm{f}$ & Transition & $\%$ \\
\hline \multicolumn{6}{|c|}{ Closed (a) } \\
\hline 3 & 4.156 & 298 & 0.833 & $\mathrm{HOMO} \rightarrow \mathrm{LUMO}+1$ & 82.0 \\
\hline 5 & 4.412 & 281 & 0.410 & HOMO-2 $\rightarrow$ LUMO & 82.6 \\
\hline 6 & 4.589 & 270 & 0.382 & $\mathrm{HOMO} \rightarrow \mathrm{LUMO}+8$ & 40.4 \\
\hline \multicolumn{6}{|c|}{ Photochemically open (b) } \\
\hline 1 & 2.692 & 461 & 1.148 & $\left\{\begin{array}{l}\text { HOMO-1 } \rightarrow \text { LUMO } \\
\text { HOMO } \rightarrow \text { LUMO }\end{array}\right.$ & $\begin{array}{l}53.3 \\
43.4\end{array}$ \\
\hline 2 & 2.805 & 442 & 0.336 & $\left\{\begin{array}{l}\text { HOMO } \rightarrow \text { LUMO } \\
\text { HOMO-1 } \rightarrow \text { LUMO }\end{array}\right.$ & $\begin{array}{l}55.3 \\
42.9\end{array}$ \\
\hline 3 & 3.623 & 342 & 0.534 & HOMO $\rightarrow$ LUMO+1 & 92.2 \\
\hline \multicolumn{6}{|c|}{ Acidly open $\left(\mathbf{b}^{+}\right)$} \\
\hline 1 & 2.648 & 468 & 1.52 & HOMO $\rightarrow$ LUMO & 96.5 \\
\hline 9 & 4.771 & 260 & 0.314 & HOMO-2 $\rightarrow$ LUMO+1 & 91.1 \\
\hline
\end{tabular}




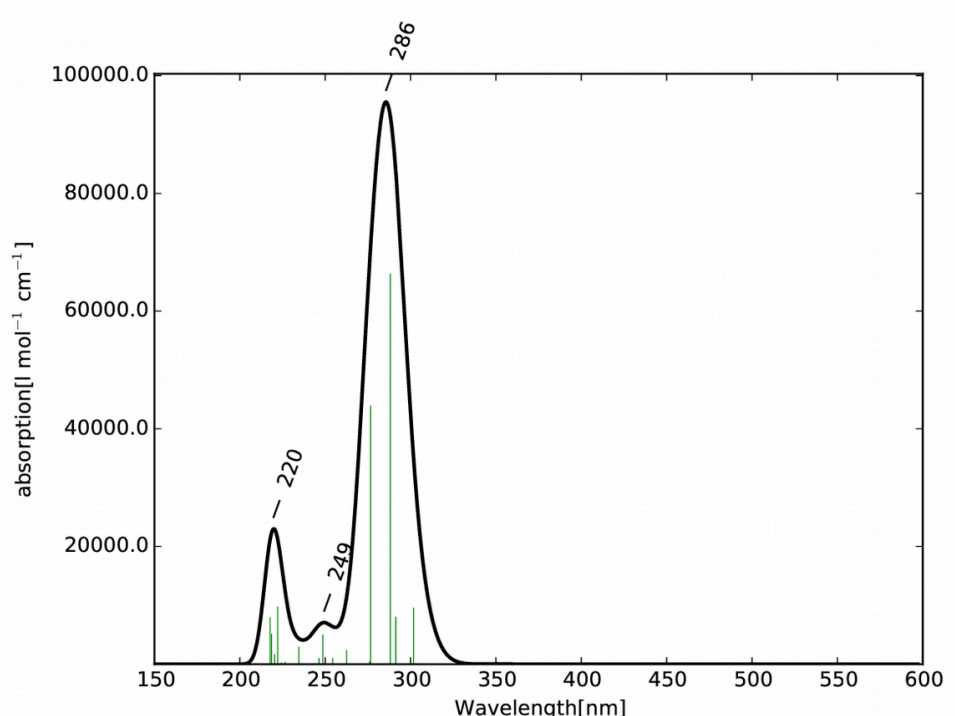

Closed (a)

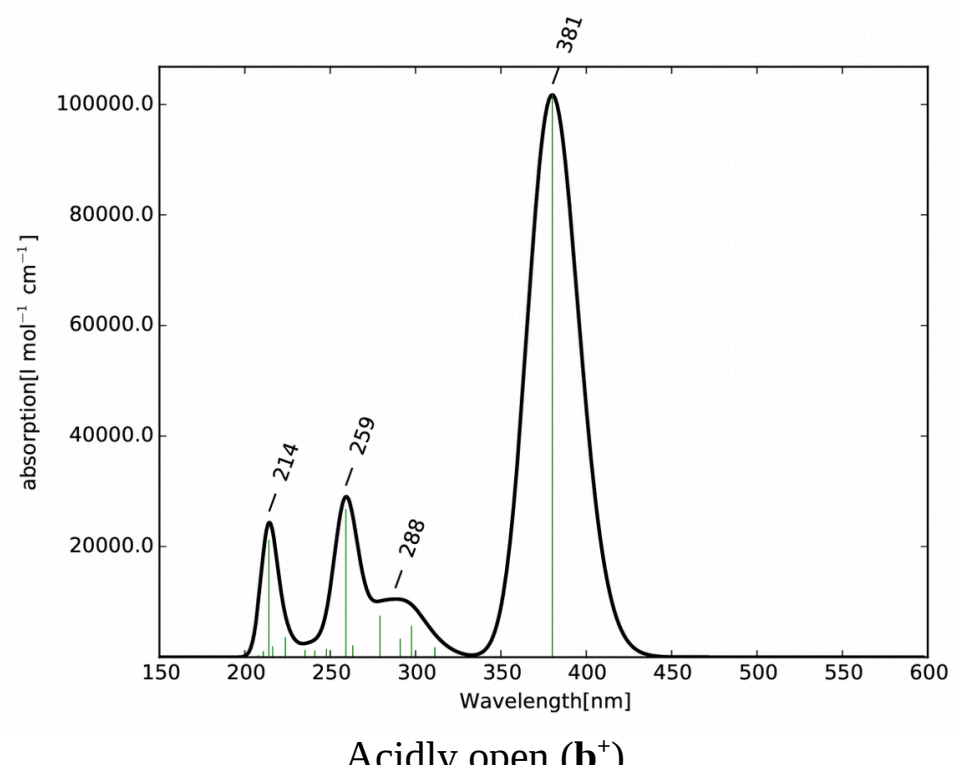

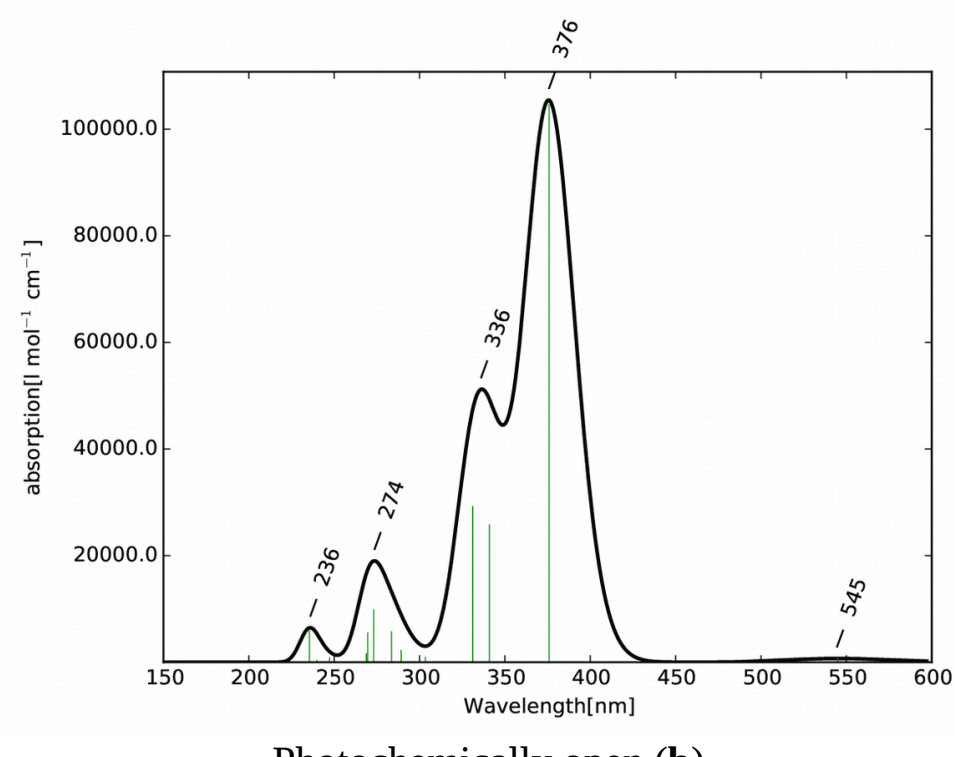

Photochemically open (b)

\begin{tabular}{|c|c|c|c|c|c|}
\hline Root & Energy $(\mathrm{eV})$ & Wavelength (nm) & $\mathrm{f}$ & Transition & $\%$ \\
\hline \multicolumn{6}{|c|}{ Closed (a) } \\
\hline 5 & 4.303 & 288 & 0.738 & $\left\{\begin{array}{l}\text { HOMO-2 } \rightarrow \text { LUMO } \\
\text { HOMO } \rightarrow \text { LUMO+1 }\end{array}\right.$ & $\begin{array}{l}42.7 \\
5.51\end{array}$ \\
\hline 6 & 4.483 & 277 & 0.488 & HOMO-1 $\rightarrow$ LUMO+1 & 69.4 \\
\hline \multicolumn{6}{|c|}{ Photochemically open (b) } \\
\hline 2 & 3.298 & 376 & 1.164 & HOMO-1 $\rightarrow$ LUMO & 93.3 \\
\hline 3 & 3.636 & 341 & 0.287 & $\mathrm{HOMO} \rightarrow \mathrm{LUMO}+2$ & 57.0 \\
\hline 4 & 3.744 & 331 & 0.325 & HOMO-2 $\rightarrow$ LUMO & 55.2 \\
\hline \multicolumn{6}{|c|}{ Acidly open $\left(\mathbf{b}^{+}\right)$} \\
\hline 1 & 3.262 & 380 & 1.132 & HOMO $\rightarrow$ LUMO & 94.1 \\
\hline 11 & 4.784 & 259 & 0.297 & HOMO-1 $\rightarrow$ LUMO+1 & 90.3 \\
\hline 18 & 5.789 & 214 & 0.236 & HOMO-1 $\rightarrow$ LUMO+3 & 48.8 \\
\hline
\end{tabular}




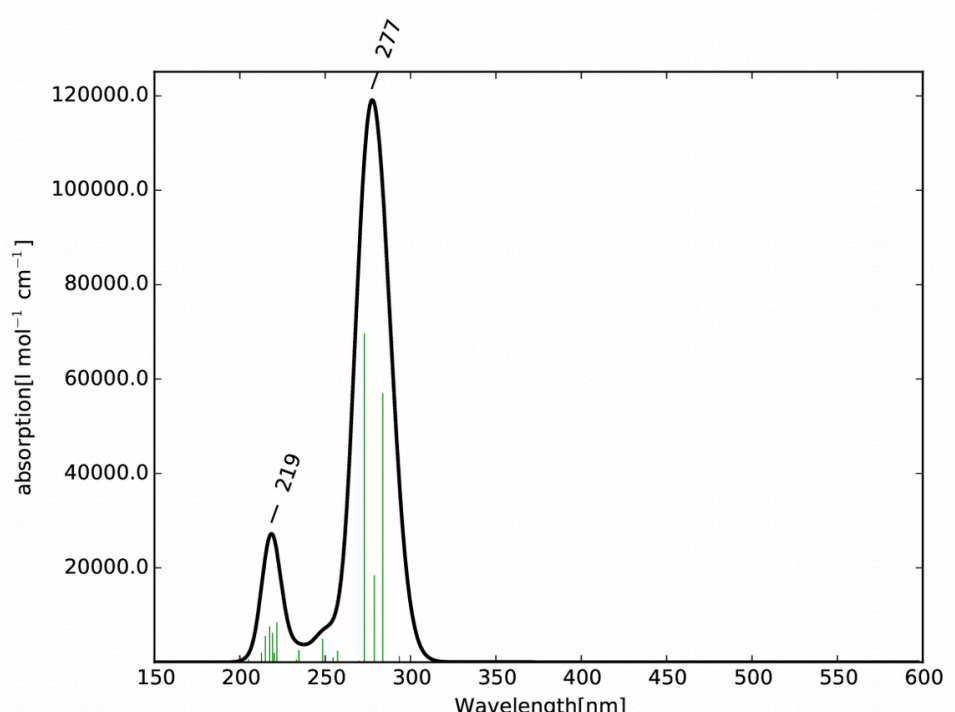

Closed (a)

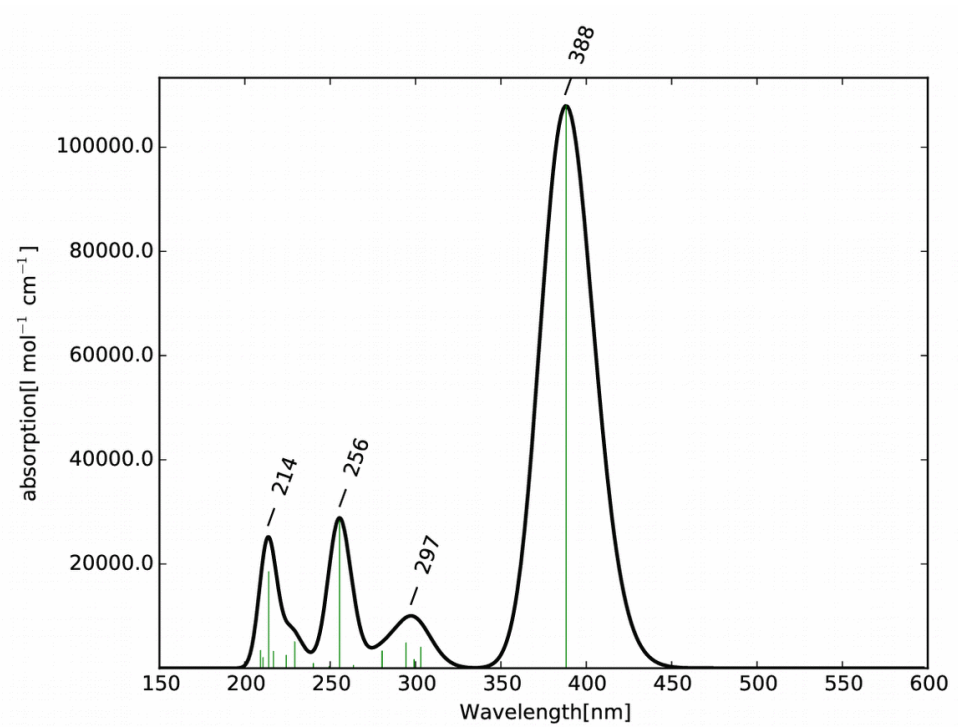

Acidly open $\left(\mathbf{b}^{+}\right)$

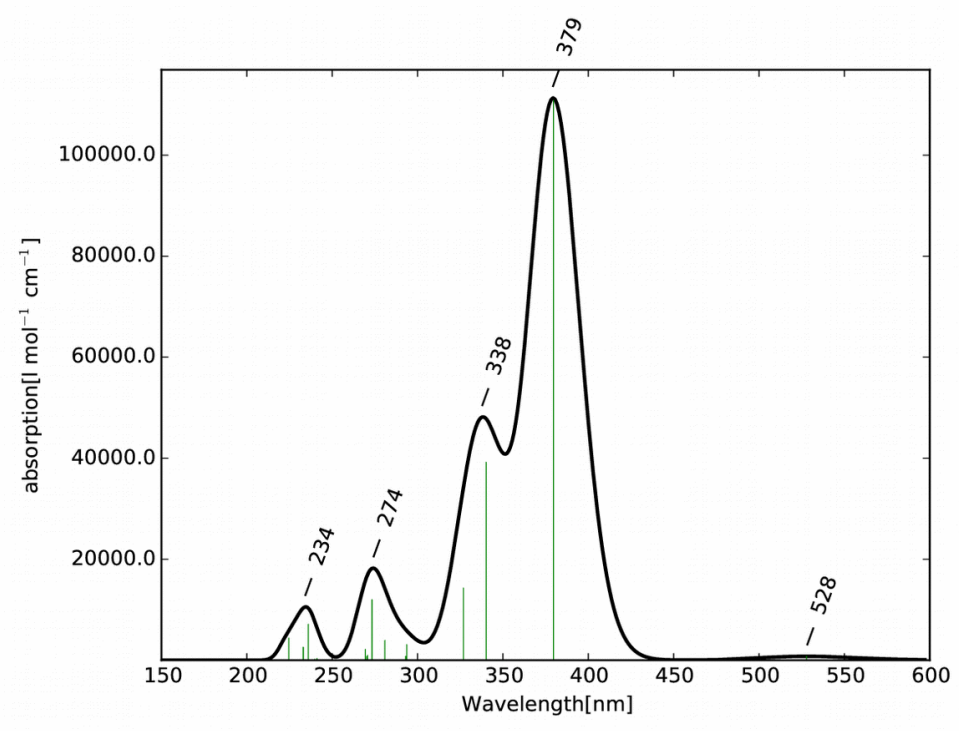

Photochemically open (b)

\begin{tabular}{cccccc}
\hline Root & Energy $(\mathrm{eV})$ & Wavelength $(\mathrm{nm})$ & $\mathrm{f}$ & Transition & $\%$ \\
\hline \multicolumn{7}{c}{ Closed $(\mathbf{a})$} \\
4 & 4.370 & 284 & 0.633 & $\left\{\begin{array}{l}\text { HOMO } \rightarrow \text { LUMO+1 } \\
\text { HOMO- } \rightarrow \text { LUMO+1 }\end{array}\right.$ & 34.2 \\
5 & 4.448 & 279 & 0.204 & HOMO $\rightarrow$ LUMO+1 & 47.7 \\
6 & 4.544 & 273 & 0.774 & HOMO-1 $\rightarrow$ LUMO+1 & 58.0 \\
\hline \multicolumn{7}{c}{ Photochemically open $(\mathbf{b})$} \\
2 & 3.266 & 380 & 1.233 & HOMO-1 $\rightarrow$ LUMO & 95.3 \\
4 & 3.645 & 340 & 0.436 & HOMO $\rightarrow$ LUMO+1 & 81.5 \\
\hline 1 & 3.194 & Acidly open $\left(\mathbf{b}^{+}\right)$ & & 95.8 \\
10 & 4.853 & 388 & 1.201 & HOMO $\rightarrow$ LUMO & 92.3 \\
18 & 5.794 & 255 & 0.315 & HOMO-1 $\rightarrow$ LUMO+1 & 48.8 \\
\hline
\end{tabular}



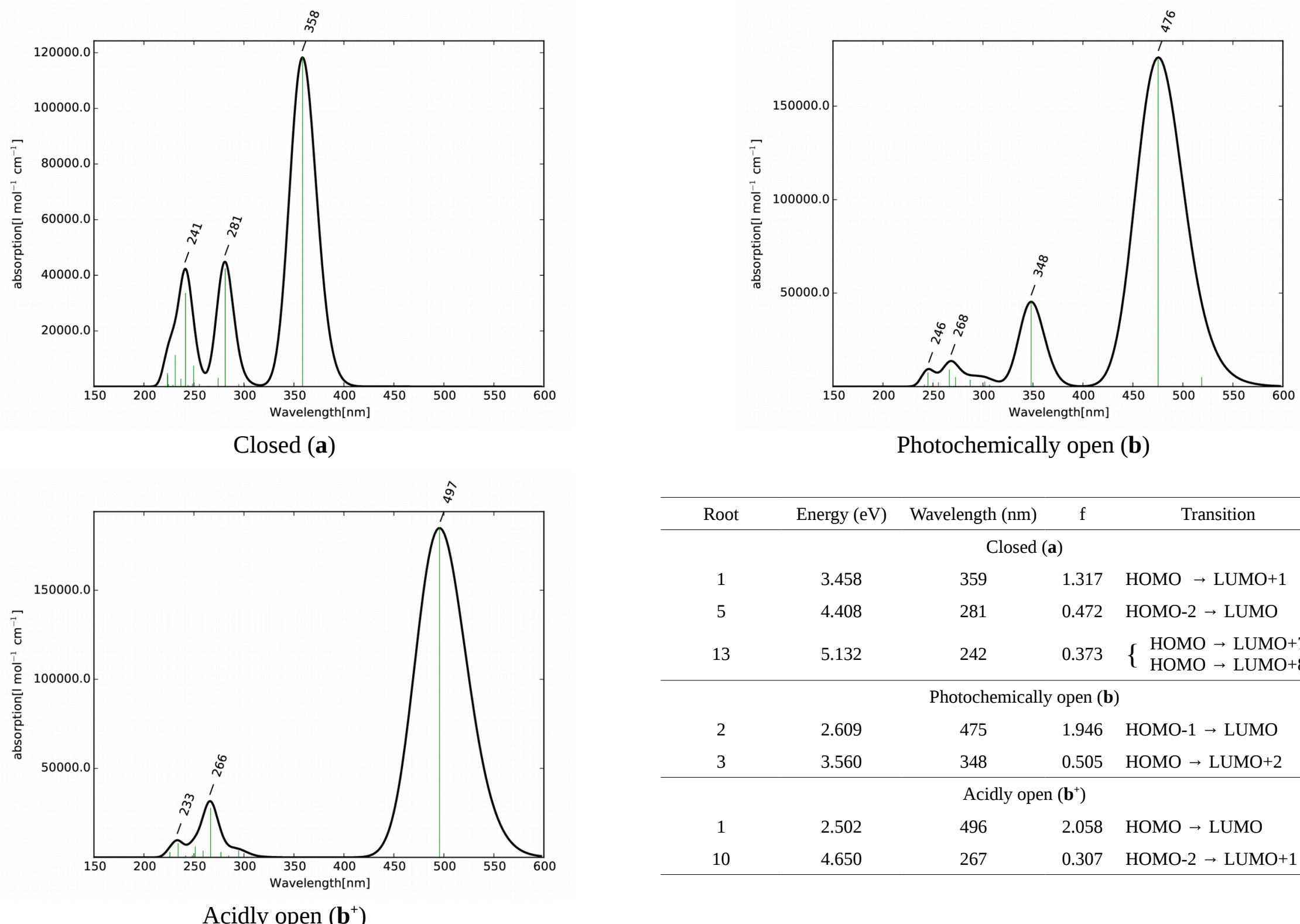

\begin{tabular}{|c|c|c|c|c|c|}
\hline Root & Energy (eV) & Wavelength (nm) & $\mathrm{f}$ & Transition & $\%$ \\
\hline \multicolumn{6}{|c|}{ Closed (a) } \\
\hline 1 & 3.458 & 359 & 1.317 & $\mathrm{HOMO} \rightarrow \mathrm{LUMO}+1$ & 94.7 \\
\hline 5 & 4.408 & 281 & 0.472 & HOMO-2 $\rightarrow$ LUMO & 84.9 \\
\hline 13 & 5.132 & 242 & 0.373 & $\left\{\begin{array}{l}\mathrm{HOMO} \rightarrow \mathrm{LUMO}+7 \\
\mathrm{HOMO} \rightarrow \mathrm{LUMO}+8\end{array}\right.$ & $\begin{array}{l}22.3 \\
11.6\end{array}$ \\
\hline \multicolumn{6}{|c|}{ Photochemically open (b) } \\
\hline 2 & 2.609 & 475 & 1.946 & HOMO-1 $\rightarrow$ LUMO & 93.1 \\
\hline 3 & 3.560 & 348 & 0.505 & $\mathrm{HOMO} \rightarrow \mathrm{LUMO}+2$ & 94.5 \\
\hline \multicolumn{6}{|c|}{ Acidly open $\left(\mathbf{b}^{+}\right)$} \\
\hline 1 & 2.502 & 496 & 2.058 & HOMO $\rightarrow$ LUMO & 94.1 \\
\hline 10 & 4.650 & 267 & 0.307 & HOMO-2 $\rightarrow$ LUMO +1 & 90.9 \\
\hline
\end{tabular}




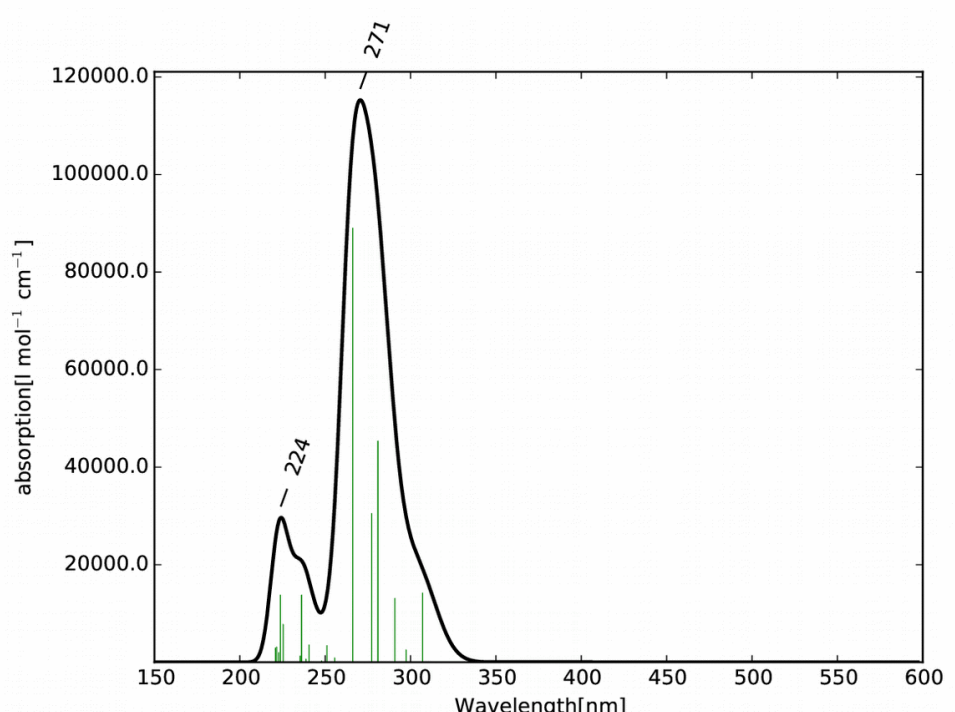

Closed (a)

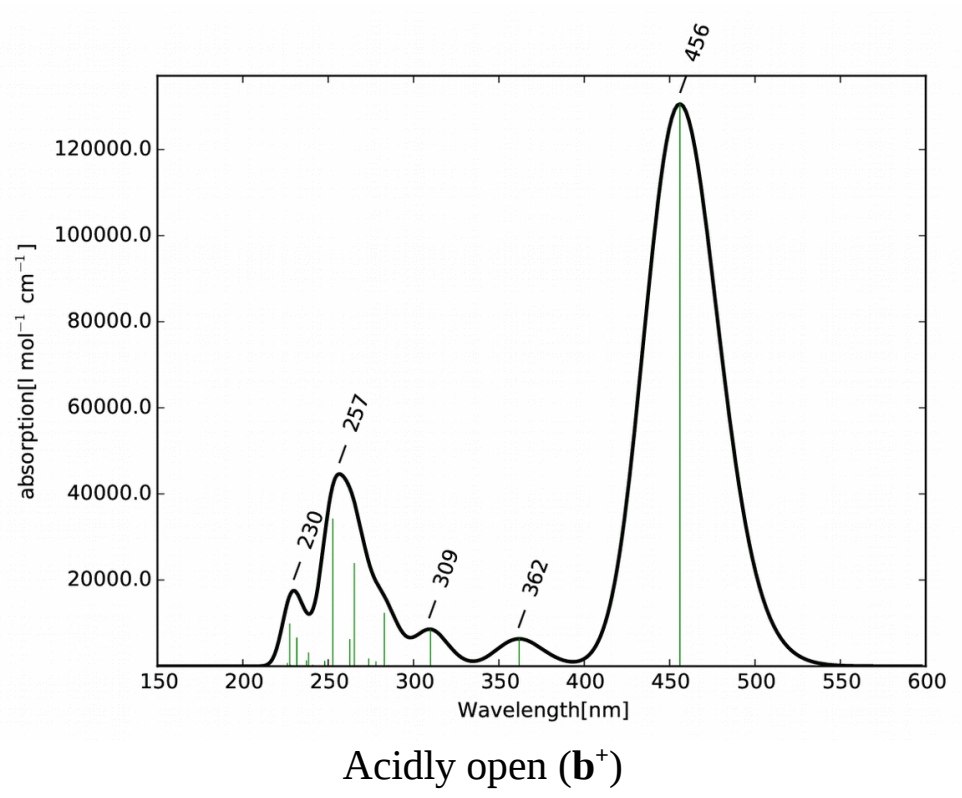

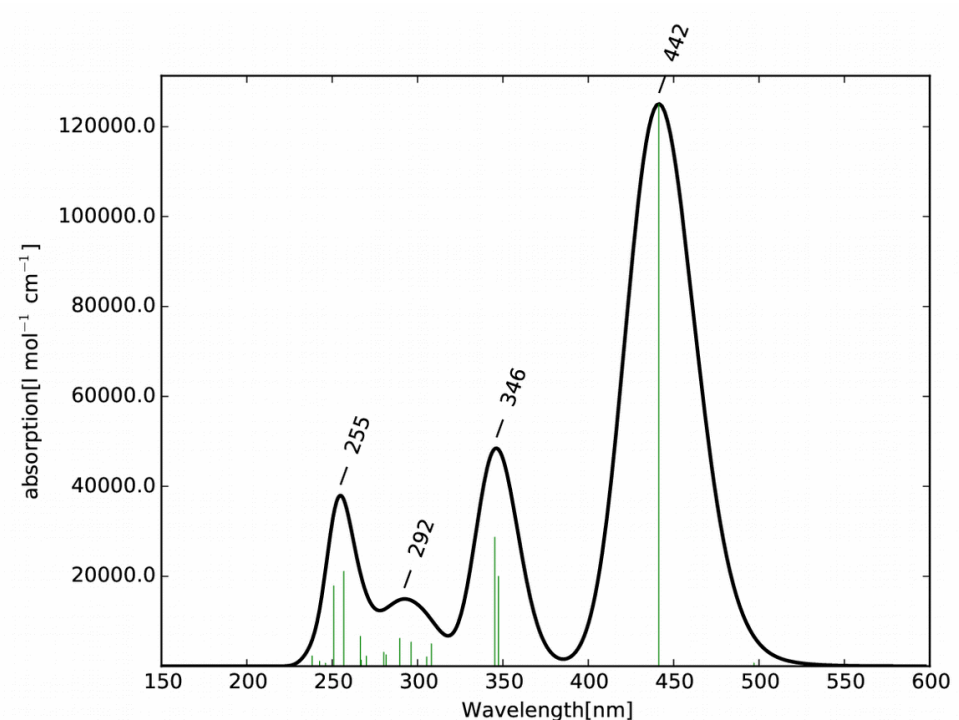

Photochemically open (b)

\begin{tabular}{|c|c|c|c|c|c|}
\hline Root & Energy $(\mathrm{eV})$ & Wavelength (nm) & $\mathrm{f}$ & Transition & $\%$ \\
\hline \multicolumn{6}{|c|}{ Closed (a) } \\
\hline 5 & 4.414 & 281 & 0.505 & $\left\{\begin{array}{l}\mathrm{HOMO}-3 \rightarrow \mathrm{LUMO} \\
\mathrm{HOMO} \rightarrow \mathrm{LUMO}+2\end{array}\right.$ & $\begin{array}{l}39.5 \\
26.1\end{array}$ \\
\hline 6 & 4.472 & 277 & 0.339 & $\mathrm{HOMO} \rightarrow \mathrm{LUMO}+2$ & 37.5 \\
\hline 8 & 4.658 & 266 & 0.990 & HOMO-2 $\rightarrow$ LUMO+1 & 70.1 \\
\hline \multicolumn{6}{|c|}{ Photochemically open (b) } \\
\hline 2 & 2.809 & 441 & 1.391 & HOMO-1 $\rightarrow$ LUMO & 92.4 \\
\hline 3 & 3.569 & 347 & 0.222 & HOMO-2 $\rightarrow$ LUMO & 51.9 \\
\hline 4 & 3.591 & 345 & 0.319 & $\mathrm{HOMO} \rightarrow \mathrm{LUMO}+1$ & 55.1 \\
\hline 15 & 4.827 & 257 & 0.235 & HOMO-7 $\rightarrow$ LUMO & 43.2 \\
\hline \multicolumn{6}{|c|}{ Acidly open $\left(\mathbf{b}^{+}\right)$} \\
\hline 1 & 2.719 & 456 & 1.453 & HOMO $\rightarrow$ LUMO & 92.8 \\
\hline 10 & 4.673 & 265 & 0.266 & HOMO-3 $\rightarrow$ LUMO+1 & 64.6 \\
\hline 12 & 4.907 & 253 & 0.380 & HOMO-1 $\rightarrow$ LUMO+3 & 71.5 \\
\hline
\end{tabular}




\section{p-nitrophenol}
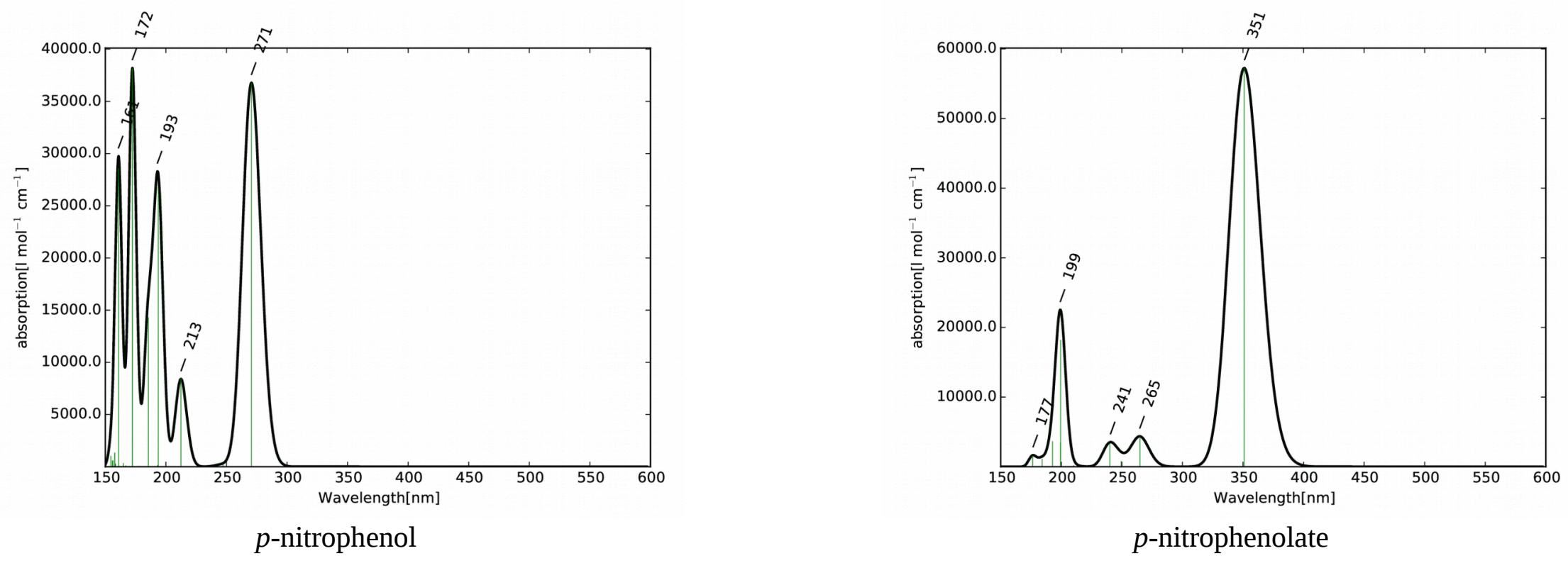

\begin{tabular}{cccclc}
\hline Root & Energy $(\mathrm{eV})$ & Wavelength $(\mathrm{nm})$ & $\mathrm{f}$ & \multicolumn{1}{c}{ Transition } & $\%$ \\
\hline \multicolumn{5}{c}{ Neutral } \\
3 & 4.581 & 271 & 0.409 & HOMO $\rightarrow$ LUMO & 96.7 \\
6 & 6.403 & 194 & 0.297 & HOMO-3 $\rightarrow$ LUMO & 87.3 \\
11 & 7.193 & 172 & 0.424 & HOMO $\rightarrow$ LUMO+5 & 55.1 \\
15 & 7.700 & 161 & 0.318 & HOMO-1 $\rightarrow$ LUMO+5 & 92.4 \\
\hline & & Negatively charged & \\
13 & 3.532 & 351 & 0.637 & HOMO $\rightarrow$ LUMO & 97.5 \\
& 6.216 & 199 & 0.202 & HOMO-4 $\rightarrow$ LUMO & 91.9 \\
\hline
\end{tabular}




\section{Closed (a)}

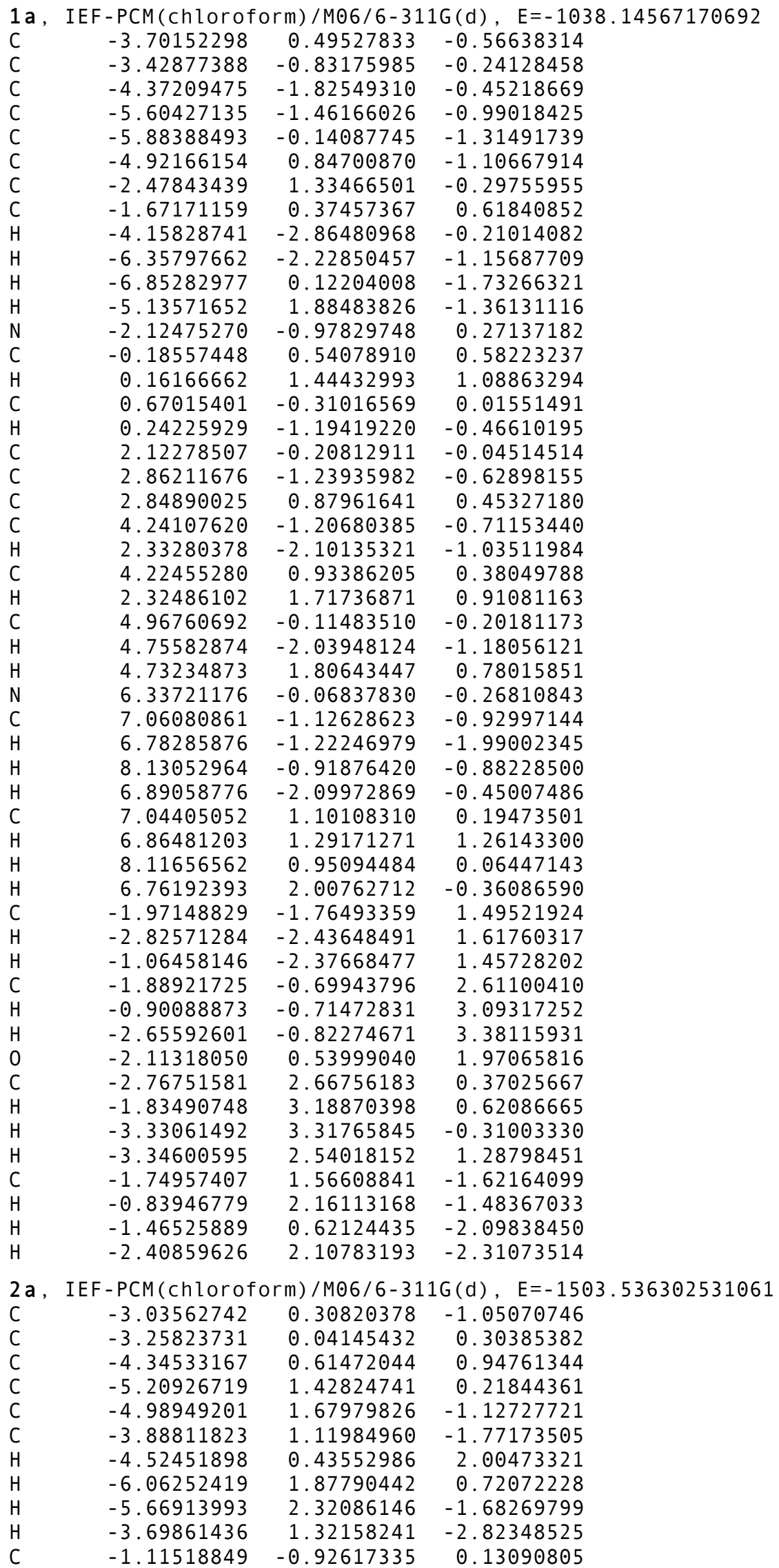




$\begin{array}{rrrr}\mathrm{C} & -0.46171103 & -2.27653521 & 0.17439991 \\ \mathrm{C} & 1.06377933 & 0.06245980 & 0.34904930 \\ \mathrm{C} & 0.87223081 & -2.34249987 & 0.09830364 \\ \mathrm{C} & 1.78919017 & 1.26737756 & 0.40499839 \\ \mathrm{C} & 1.69319090 & -1.14950633 & 0.06709209 \\ \mathrm{H} & 1.37025952 & -3.31055437 & 0.05458842 \\ \mathrm{C} & 3.15049653 & 1.23612275 & 0.15704148 \\ \mathrm{C} & 3.06405637 & -1.17169709 & -0.17642770 \\ \mathrm{C} & 3.76000742 & 0.01730829 & -0.13159738 \\ \mathrm{H} & 3.75663173 & 2.13399540 & 0.17421961 \\ \mathrm{H} & 3.57847978 & -2.10324817 & -0.39016740 \\ \mathrm{~S} & -1.59611023 & -0.53640630 & -1.63289641 \\ \mathrm{~N} & -2.33162371 & -0.83044940 & 0.86614352 \\ \mathrm{O} & -0.24885583 & 0.11337202 & 0.62630749 \\ \mathrm{C} & -1.35268575 & -3.46870547 & 0.17948597 \\ \mathrm{H} & -1.97572167 & -3.50726985 & 1.08082948 \\ \mathrm{H} & -2.04482867 & -3.44471192 & -0.67092432 \\ \mathrm{H} & -0.76794916 & -4.39097200 & 0.12447018 \\ \mathrm{C} & -2.25528263 & -0.96600374 & 2.30657437 \\ \mathrm{H} & -2.05671673 & -0.00502949 & 2.79997370 \\ \mathrm{H} & -3.19330869 & -1.37727095 & 2.69253474 \\ \mathrm{H} & -1.44984293 & -1.65656622 & 2.56771717 \\ \mathrm{~N} & 5.20093264 & 0.00506967 & -0.38846245 \\ \mathrm{O} & 1.08092980 & 2.36797342 & 0.69873730 \\ \mathrm{C} & 1.77327083 & 3.59724775 & 0.75561954 \\ \mathrm{H} & 2.53712923 & 3.58853122 & 1.54281511 \\ \mathrm{H} & 2.24465458 & 3.83385586 & -0.20624388 \\ \mathrm{H} & 1.02656516 & 4.35616401 & 0.98606534 \\ \mathrm{O} & 5.72667375 & -1.06330381 & -0.63519580 \\ \mathrm{O} & 5.79754764 & 1.06412638 & -0.34065000\end{array}$

3a , IEF-PCM (chlor oform) /M06/6-311G(d), E=-1621.156568718205

$\begin{array}{lrrr}\text { C } & -2.06645419 & -1.86397527 & -0.73182271 \\ \mathrm{C} & -1.75312231 & -2.79195990 & 0.26286590 \\ \mathrm{C} & -2.72402893 & -3.62756557 & 0.77036069 \\ \mathrm{C} & -4.02989441 & -3.52252161 & 0.28572289 \\ \mathrm{C} & -4.33358629 & -2.59010498 & -0.69553573 \\ \mathrm{C} & -3.35652068 & -1.74209331 & -1.21860152 \\ \mathrm{C} & 0.03597399 & -1.30281449 & -0.02321499 \\ \mathrm{C} & -0.27222189 & -2.71760293 & 0.55038178 \\ \mathrm{H} & -2.47963502 & -4.35543128 & 1.54307939 \\ \mathrm{H} & -4.81010210 & -4.16820236 & 0.68105273 \\ \mathrm{H} & -5.35412891 & -2.50821101 & -1.06307158 \\ \mathrm{H} & -3.61598557 & -1.01272160 & -1.98239022 \\ \mathrm{~N} & -0.90339464 & -1.17339753 & -1.11491456 \\ \mathrm{C} & 0.44505382 & -3.79366250 & -0.26728589 \\ \mathrm{H} & 1.52853540 & -3.76581052 & -0.10120296 \\ \mathrm{H} & 0.08384397 & -4.78048611 & 0.04288498 \\ \mathrm{H} & 0.25516890 & -3.68950555 & -1.34106047 \\ \mathrm{C} & 0.10406096 & -2.86282387 & 2.01318420 \\ \mathrm{H} & -0.06285598 & -3.89632746 & 2.33750008 \\ \mathrm{H} & 1.16812254 & -2.64085996 & 2.16271515 \\ \mathrm{H} & -0.47997581 & -2.20368813 & 2.65903295 \\ \mathrm{O} & -0.30487288 & -0.36912485 & 1.04145259 \\ \mathrm{C} & -0.97380862 & 0.15411994 & -1.69026733 \\ \mathrm{H} & -1.80259129 & 0.20177192 & -2.40321905 \\ \mathrm{H} & -0.06002698 & 0.33512487 & -2.27280510 \\ \mathrm{C} & -1.12103256 & 1.18665253 & -0.61211776 \\ \mathrm{C} & -0.75855870 & 0.84840567 & 0.69962173 \\ \mathrm{C} & -0.87324366 & 1.78233430 & 1.73310632 \\ \mathrm{H} & -0.58743877 & 1.48338942 & 2.73779892 \\ \mathrm{C} & -1.58728837 & 2.46304503 & -0.86543666 \\ \mathrm{H} & -1.87899526 & 2.75036492 & -1.87207426 \\ \mathrm{C} & -1.33681047 & 3.05145480 & 1.47165542 \\ \mathrm{C} & -1.68764534 & 3.38234167 & 0.16718793 \\ \mathrm{~N} & -2.18115014 & 4.72289814 & -0.12044095 \\ & & & \end{array}$




$\begin{array}{rrrr}0 & -2.26820110 & 5.51181984 & 0.80291568 \\ \mathrm{O} & -2.48120002 & 4.98989003 & -1.27007650 \\ \mathrm{H} & -1.42951243 & 3.78928451 & 2.26090311 \\ \mathrm{C} & 1.44389243 & -1.06430258 & -0.45747082 \\ \mathrm{H} & 1.72023432 & -1.55719239 & -1.39002445 \\ \mathrm{C} & 2.33332808 & -0.33654987 & 0.22131091 \\ \mathrm{H} & 2.00895121 & 0.15358394 & 1.14048455 \\ \mathrm{C} & 3.71793553 & -0.14202494 & -0.15889094 \\ \mathrm{C} & 4.45531924 & -0.73310371 & -1.15238755 \\ \mathrm{C} & 6.06765561 & 0.66018674 & -0.25290890 \\ \mathrm{H} & 4.05110940 & -1.48751341 & -1.82155628 \\ \mathrm{C} & 5.79105771 & -0.27429289 & -1.20648652 \\ \mathrm{H} & 7.00162323 & 1.17449954 & -0.06449697 \\ \mathrm{H} & 6.52501144 & -0.62801875 & -1.92365224 \\ \mathrm{~S} & 4.69526815 & 0.99499461 & 0.72909071\end{array}$

4a, IEF-PCM (chloroform) /MO6/6-311G(d), E=-1621.157658923725

\begin{tabular}{|c|c|c|c|}
\hline C & -2.09197918 & -1.85922927 & -0.75197970 \\
\hline $\mathrm{C}$ & -1.79616929 & -2.79125890 & ๑. 24358990 \\
\hline C & -2.77266891 & -3.63794857 & 0.72038972 \\
\hline C & -4.06616840 & -3.54043860 & ๑. 20314992 \\
\hline C & -4.35249828 & -2.60394897 & -0.77891969 \\
\hline C & -3.36976867 & -1.74452931 & -1.27069950 \\
\hline C & $-\odot .011450 \odot \odot$ & -1.28654949 & $\odot .012 \odot 8 \odot \odot \odot$ \\
\hline C & -0.32324987 & -2.70767894 & $\odot .56732978$ \\
\hline $\mathrm{H}$ & -2.54210900 & -4.36843828 & 1.49509941 \\
\hline $\mathrm{H}$ & -4.85106809 & -4.19477835 & ๑. 57421977 \\
\hline $\mathrm{H}$ & -5.36416787 & -2.52764900 & -1.17138954 \\
\hline $\mathrm{H}$ & -3.61545857 & -1.01085960 & -2.03516920 \\
\hline $\mathrm{N}$ & -0.92516964 & -1.15654954 & -1.10125957 \\
\hline C & 0.42114983 & -3.77211851 & -0.24109991 \\
\hline $\mathrm{H}$ & 1. 50060941 & -3.73591853 & -0.05184998 \\
\hline $\mathrm{H}$ & $\odot .06182998$ & -4.76434812 & 0.05379998 \\
\hline $\mathrm{H}$ & ๑. 25179990 & -3.66263855 & -1.31774948 \\
\hline C & $\odot .01849999$ & -2.86023887 & 2.03767920 \\
\hline $\mathrm{H}$ & -0.14894994 & -3.89702846 & 2.35164907 \\
\hline $\mathrm{H}$ & 1.07697958 & -2.63177896 & 2.21460913 \\
\hline $\mathrm{H}$ & -0.58638977 & -2.20908913 & 2.67223895 \\
\hline 0 & $-\odot .38437985$ & $-\odot .36433986$ & 1.07471958 \\
\hline C & -0.99320961 & ๑.17397993 & -1.66817934 \\
\hline $\mathrm{H}$ & -1.81132929 & ๑.21978991 & -2.39399906 \\
\hline $\mathrm{H}$ & $-\odot .07094997$ & $\odot .36440986$ & -2.23488912 \\
\hline C & -1.16495954 & 1. 20008952 & -0.58755977 \\
\hline C & -0.83160967 & $\odot .85466966$ & ๑. 72964971 \\
\hline C & -0.97062962 & 1.78354929 & 1.76435930 \\
\hline $\mathrm{H}$ & $-\odot .70990972$ & 1.47782942 & 2.77392891 \\
\hline C & -1.62471936 & 2.47818903 & -0.84490967 \\
\hline $\mathrm{H}$ & -1.89358925 & 2.77296890 & -1.85592927 \\
\hline C & -1.42751944 & 3.05449879 & 1.49876941 \\
\hline C & -1.74818931 & 3.39229866 & ๑.18889993 \\
\hline $\mathrm{N}$ & -2.23410912 & 4.73685813 & -0.10416996 \\
\hline 0 & -2.33187908 & 5.52198782 & 0.82014968 \\
\hline 0 & -2.51482901 & $5.0 \odot 4 \odot 5803$ & -1.25786950 \\
\hline $\mathrm{H}$ & -1.53931939 & 3.78993851 & 2.28794910 \\
\hline C & 1.40492945 & -1.03406959 & -0.38811985 \\
\hline $\mathrm{H}$ & 1.69835933 & -1.51307941 & -1.32284948 \\
\hline C & 2.27768910 & -0.31565988 & ๑. 31947987 \\
\hline $\mathrm{H}$ & 1.93915924 & $\odot .14540994$ & 1. 24886951 \\
\hline C & 3.67007856 & -0.08398997 & -0.02680999 \\
\hline C & 4.50540823 & $\odot .66295974$ & $\odot .76101970$ \\
\hline C & 4.34876828 & $-\odot .57875977$ & -1.18440953 \\
\hline$S$ & 6.09585757 & $\odot .77214969$ & ๑. 11956995 \\
\hline $\mathrm{H}$ & 4.26020832 & 1.15720954 & 1.69387933 \\
\hline $\mathrm{C}$ & 5.64936776 & $-\odot .20007992$ & -1.23846951 \\
\hline $\mathrm{H}$ & 3.88132847 & -1.19374953 & -1.94762923 \\
\hline $\mathrm{H}$ & 6.38629748 & -0.43089983 & -1.9973192 \\
\hline
\end{tabular}


5a, IEF-PCM (chloroform) /MO6/6-311G(d), E=-1817.517521564118

\begin{tabular}{|c|c|c|c|}
\hline C & 4.89641367 & -1.51737881 & 1. 00990487 \\
\hline C & 4.80037202 & $-2.590 \odot 8048$ & ๑. 12206992 \\
\hline C & 5.91080570 & -3.34605674 & -0.18244709 \\
\hline C & 7.13728500 & -3.01446126 & ๑. 39745678 \\
\hline C & 7. 22476008 & -1.93999496 & 1. 26952707 \\
\hline C & 6.10507330 & -1.17112981 & 1.58787427 \\
\hline C & 2.83214578 & -1.31720973 & 0.04248926 \\
\hline C & 3.36043597 & -2.73863743 & -0.30894712 \\
\hline $\mathrm{H}$ & 5.83641252 & -4.18563752 & -0.87263111 \\
\hline $\mathrm{H}$ & 8.02554865 & -3.59449507 & $\odot .15976589$ \\
\hline $\mathrm{H}$ & 8.18516394 & -1.68116386 & 1.70994217 \\
\hline $\mathrm{H}$ & 6.19581186 & -0.32584317 & 2.26608863 \\
\hline $\mathrm{N}$ & 3.62584835 & $-\odot .94361774$ & 1.19278470 \\
\hline C & 2.68481176 & -3.78994637 & $\odot .57344194$ \\
\hline $\mathrm{H}$ & 1.62831883 & -3.91571437 & ๑.30799360 \\
\hline $\mathrm{H}$ & 3.18352253 & -4.75520521 & $\odot .43140526$ \\
\hline $\mathrm{H}$ & 2.74896423 & -3.53450518 & 1.63662341 \\
\hline C & 3.16591656 & -3.10222566 & -1.76904610 \\
\hline $\mathrm{H}$ & 3.48065600 & -4.13848160 & -1.93816915 \\
\hline $\mathrm{H}$ & 2.10706932 & -3.03276799 & -2.04821096 \\
\hline $\mathrm{H}$ & 3.74259352 & -2.45675269 & -2.43500142 \\
\hline 0 & 3.18364090 & -0.47950424 & -1.09620827 \\
\hline C & 3.47543891 & 0.43803221 & 1.59880917 \\
\hline $\mathrm{H}$ & 4.21655598 & ๑.67599131 & 2.36871726 \\
\hline $\mathrm{H}$ & 2.49190603 & 0.56014859 & 2.07399982 \\
\hline C & 3.60431462 & 1.35188090 & 0.41657575 \\
\hline C & 3.44024787 & 0.81978069 & -0.87004691 \\
\hline C & 3.56018258 & 1.63798597 & -1.99709418 \\
\hline $\mathrm{H}$ & 3.43635425 & 1.18879119 & -2.97876286 \\
\hline C & 3.87314599 & 2.70108416 & ๑. 55085143 \\
\hline $\mathrm{H}$ & 4.00761572 & 3.13901887 & 1.53643678 \\
\hline C & 3.82881338 & 2.98027427 & -1.85404289 \\
\hline C & 3.97753377 & 3.50315429 & -0.57446398 \\
\hline $\mathrm{N}$ & 4.25840193 & 4.92617413 & -0.41277913 \\
\hline 0 & 4.35478146 & 5.60573759 & -1.41738594 \\
\hline 0 & 4.38010916 & 5.36053047 & $\odot .71766994$ \\
\hline $\mathrm{H}$ & 3.92204186 & 3.63029524 & -2.71691868 \\
\hline C & 1.36738204 & -1.20350648 & ๑. 31089012 \\
\hline $\mathrm{H}$ & 1.06246910 & -1.59464485 & 1. 28192807 \\
\hline C & $\odot .47809327$ & -0.68904869 & -0.54021770 \\
\hline $\mathrm{H}$ & $\odot .84935718$ & $-\odot .31091486$ & -1.49500947 \\
\hline C & -0.96143801 & $-\odot .56458211$ & -0.34325198 \\
\hline C & -1.62511845 & $-1 . \odot \odot \odot 14531$ & $\odot .81018411$ \\
\hline C & -1.73535838 & $\odot .02336383$ & -1.34738069 \\
\hline C & -2.98941555 & $-\odot .85419880$ & $\odot .95119709$ \\
\hline $\mathrm{H}$ & -1.06747584 & -1.47872943 & 1.61297921 \\
\hline C & -3.10229650 & ๑. 18269729 & -1.21325920 \\
\hline $\mathrm{H}$ & -1.24548567 & ๑. 37963981 & -2.25264785 \\
\hline C & -3.75282668 & $-\odot .25515314$ & -0.05867367 \\
\hline $\mathrm{H}$ & -3.48431593 & -1.21235918 & 1.85111357 \\
\hline $\mathrm{H}$ & -3.67607397 & ๑.65702913 & -2.00600371 \\
\hline $\mathrm{N}$ & -5.14232337 & -0.10439393 & $\odot .08714814$ \\
\hline C & -5.69846544 & ๑. 23952599 & 1.34187047 \\
\hline C & -5.09504473 & 1.20911420 & 2.14306197 \\
\hline C & -6.86686349 & -0.37925163 & 1.78638182 \\
\hline C & -5.64683740 & 1.54243018 & 3.36999762 \\
\hline $\mathrm{H}$ & -4.18982162 & 1.70150398 & 1.79400269 \\
\hline C & -7.42245573 & $-\odot .02764237$ & $3.0 \odot 636564$ \\
\hline $\mathrm{H}$ & -7.33690358 & -1.13895328 & 1.16553172 \\
\hline C & -6.81499552 & $\odot .93115106$ & 3.80710782 \\
\hline $\mathrm{H}$ & -5.16566055 & 2.30088399 & 3.98340526 \\
\hline $\mathrm{H}$ & -8.33427131 & $-\odot .51821960$ & 3.33948007 \\
\hline $\mathrm{H}$ & -7.25005643 & 1. 20123305 & 4.76605699 \\
\hline C & -5.99540713 & -0.25460638 & -1.03329617 \\
\hline C & -5.83166320 & -1.33142505 & -1.90432967 \\
\hline
\end{tabular}




$\begin{array}{lrrr}\mathrm{C} & -7.01301688 & 0.66779064 & -1.27345520 \\ \mathrm{C} & -6.66697344 & -1.47450707 & -3.00086581 \\ \mathrm{H} & -5.04475957 & -2.05788951 & -1.71269789 \\ \mathrm{C} & -7.85527860 & 0.50909174 & -2.36287356 \\ \mathrm{H} & -7.13844220 & 1.51038493 & -0.59668436 \\ \mathrm{C} & -7.68499320 & -0.55848776 & -3.23464003 \\ \mathrm{H} & -6.52930286 & -2.31949181 & -3.67171450 \\ \mathrm{H} & -8.64472977 & 1.23640764 & -2.53830897 \\ \mathrm{H} & -8.34314577 & -0.67689489 & -4.09174227\end{array}$

$6 \mathrm{a}, \mathrm{IEF}-\mathrm{PCM}(\mathrm{chl}$ or of orm) $/$ M06/6-311G(d), E=-1223.003388217701

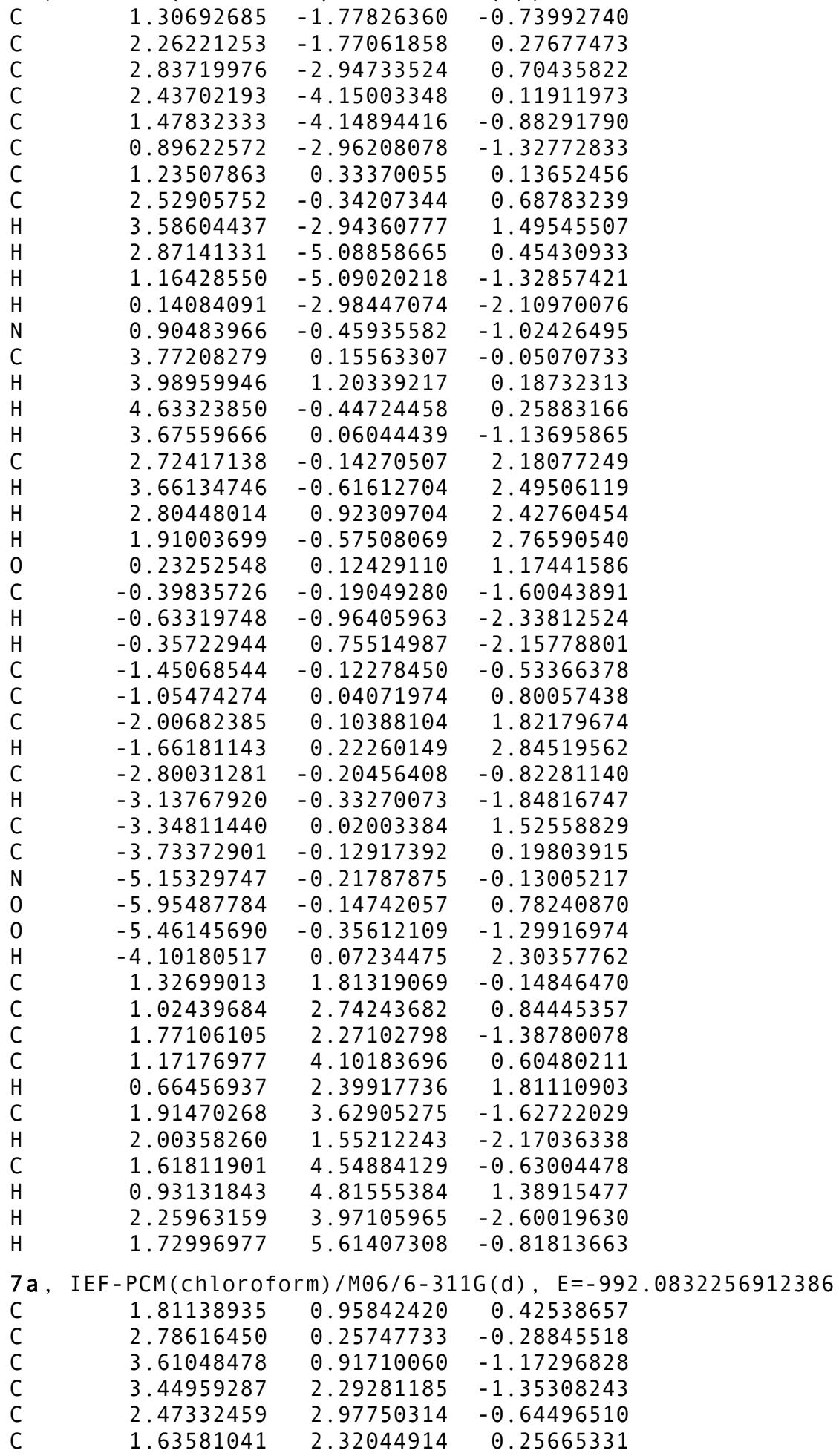




$\begin{array}{lrrr}\mathrm{C} & 1.35569538 & -1.24523936 & 0.78315163 \\ \mathrm{C} & 2.75070532 & -1.19278097 & 0.13991182 \\ \mathrm{H} & 4.37315315 & 0.37495404 & -1.73120275 \\ \mathrm{H} & 4.08598326 & 2.82710197 & -2.05402100 \\ \mathrm{H} & 2.34888573 & 4.04733736 & -0.79736715 \\ \mathrm{H} & 0.86959736 & 2.87139478 & 0.79743492 \\ \mathrm{~N} & 1.14540112 & 0.07513764 & 1.29262923 \\ \mathrm{C} & 3.79585990 & -1.43200790 & 1.23043353 \\ \mathrm{H} & 3.75224235 & -2.46642467 & 1.59381641 \\ \mathrm{H} & 4.80047335 & -1.25607310 & 0.83025555 \\ \mathrm{H} & 3.64949999 & -0.76070735 & 2.08331522 \\ \mathrm{C} & 2.94510825 & -2.18665785 & -0.99016710 \\ \mathrm{H} & 3.97965229 & -2.14074540 & -1.34932573 \\ \mathrm{H} & 2.76252014 & -3.21239162 & -0.64775161 \\ \mathrm{H} & 2.28307363 & -1.98378688 & -1.83513479 \\ \mathrm{O} & 0.39049673 & -1.58444716 & -0.24572994 \\ \mathrm{C} & -0.18753949 & 0.33675652 & 1.78922090 \\ \mathrm{H} & -0.29340662 & 1.40254190 & 2.01654258 \\ \mathrm{H} & -0.31563195 & -0.19261155 & 2.74385204 \\ \mathrm{C} & -1.21387535 & -0.11170952 & 0.79070302 \\ \mathrm{C} & -0.84328634 & -1.06381472 & -0.17059231 \\ \mathrm{C} & -1.76827600 & -1.50640176 & -1.12092887 \\ \mathrm{H} & -1.44516658 & -2.23917334 & -1.85493501 \\ \mathrm{C} & -2.50667471 & 0.37569045 & 0.78515498 \\ \mathrm{H} & -2.81841264 & 1.11924741 & 1.51400586 \\ \mathrm{C} & -3.05589350 & -1.02073610 & -1.11509964 \\ \mathrm{C} & -3.41590574 & -0.08051494 & -0.15589955 \\ \mathrm{~N} & -4.77624301 & 0.44590535 & -0.14466206 \\ \mathrm{O} & -5.56033336 & 0.01639982 & -0.97009133 \\ \mathrm{O} & -5.05757085 & 1.28603328 & 0.69008909 \\ \mathrm{H} & -3.78674067 & -1.35654876 & -1.84255402 \\ \mathrm{H} & 1.25332475 & -2.00340400 & 1.57241285\end{array}$

8a, IEF-PCM (chloroform) /M06/6-311G(d), E=-1434.259018644183

$\begin{array}{lrrr}\mathrm{C} & -2.96975019 & -1.69179543 & -0.82431886 \\ \mathrm{C} & -2.85812501 & -2.63739999 & 0.19661650 \\ \mathrm{C} & -3.95424618 & -3.37505767 & 0.58669602 \\ \mathrm{C} & -5.18141512 & -3.15149812 & -0.04112076 \\ \mathrm{C} & -5.28533339 & -2.19930355 & -1.04439355 \\ \mathrm{C} & -4.18043545 & -1.45137219 & -1.45064468 \\ \mathrm{C} & -0.91106649 & -1.32479637 & 0.11359827 \\ \mathrm{C} & -1.41990226 & -2.69359965 & 0.65419933 \\ \mathrm{H} & -3.86851032 & -4.11798794 & 1.37923184 \\ \mathrm{H} & -6.05822383 & -3.71859423 & 0.26167258 \\ \mathrm{H} & -6.24721220 & -2.02294903 & -1.52099439 \\ \mathrm{H} & -4.28321432 & -0.70300020 & -2.23320230 \\ \mathrm{~N} & -1.71112209 & -1.12077106 & -1.07635563 \\ \mathrm{C} & -0.71465008 & -3.84515105 & -0.06400690 \\ \mathrm{H} & 0.34185960 & -3.90642697 & 0.22323640 \\ \mathrm{H} & -1.19472899 & -4.79073644 & 0.21193262 \\ \mathrm{H} & -0.77386624 & -3.74570086 & -1.15323697 \\ \mathrm{C} & -1.23801428 & -2.84622514 & 2.15308123 \\ \mathrm{H} & -1.53136287 & -3.85639946 & 2.46153764 \\ \mathrm{H} & -0.18561994 & -2.71107935 & 2.43316401 \\ \mathrm{H} & -1.83985914 & -2.12930122 & 2.71570248 \\ \mathrm{O} & -1.27449648 & -0.34587624 & 1.13049110 \\ \mathrm{C} & -1.58870023 & 0.19991147 & -1.65610254 \\ \mathrm{H} & -2.30711693 & 0.31260042 & -2.47443601 \\ \mathrm{H} & -0.59049467 & 0.28994453 & -2.10680463 \\ \mathrm{C} & -1.78607205 & 1.25128667 & -0.60541125 \\ \mathrm{C} & -1.59312706 & 0.89753182 & 0.73873305 \\ \mathrm{C} & -1.75354537 & 1.84761483 & 1.75140298 \\ \mathrm{H} & -1.59887835 & 1.53798277 & 2.78136838 \\ \mathrm{C} & -2.14427339 & 2.55115407 & -0.90808766 \\ \mathrm{H} & -2.31080894 & 2.85093226 & -1.93955829 \\ \mathrm{C} & -2.10469598 & 3.14160340 & 1.44019206\end{array}$




$\begin{array}{lrrr}\mathrm{C} & -2.29842329 & 3.48292591 & 0.10636406 \\ \mathrm{~N} & -2.68194039 & 4.84811174 & -0.23524320 \\ \mathrm{O} & -2.80058494 & 5.65195888 & 0.67073293 \\ \mathrm{O} & -2.86391412 & 5.11369146 & -1.40920373 \\ \mathrm{H} & -2.23329686 & 3.89126232 & 2.21325521 \\ \mathrm{C} & 0.54947956 & -1.22295088 & -0.17491536 \\ \mathrm{H} & 0.86465027 & -1.78066083 & -1.05749155 \\ \mathrm{C} & 1.42842123 & -0.52049516 & 0.54402527 \\ \mathrm{H} & 1.05281564 & 0.03688695 & 1.40517710 \\ \mathrm{C} & 2.85907828 & -0.39490849 & 0.30567416 \\ \mathrm{C} & 3.55026495 & -1.11734335 & -0.67357523 \\ \mathrm{C} & 3.61083781 & 0.49555565 & 1.07628975 \\ \mathrm{C} & 4.90316884 & -0.95652303 & -0.87927331 \\ \mathrm{H} & 3.01787674 & -1.83486809 & -1.29612013 \\ \mathrm{C} & 4.96706410 & 0.67433370 & 0.88611958 \\ \mathrm{H} & 3.10868203 & 1.07591517 & 1.85015170 \\ \mathrm{C} & 5.65822507 & -0.05052484 & -0.10328318 \\ \mathrm{H} & 5.38331921 & -1.54516620 & -1.65499323 \\ \mathrm{H} & 5.49229295 & 1.38704580 & 1.51445469 \\ \mathrm{~N} & 7.00400283 & 0.11101189 & -0.30367006 \\ \mathrm{C} & 7.66314650 & -0.58623846 & -1.38199897 \\ \mathrm{H} & 7.58350753 & -1.67578085 & -1.27183291 \\ \mathrm{H} & 7.25257203 & -0.31665664 & -2.36611605 \\ \mathrm{H} & 8.72389973 & -0.33327093 & -1.38203962 \\ \mathrm{C} & 7.72967722 & 1.10190331 & 0.45400629 \\ \mathrm{H} & 8.77987718 & 1.08647113 & 0.16047143 \\ \mathrm{H} & 7.34669865 & 2.11891614 & 0.28417042 \\ \mathrm{H} & 7.68499695 & 0.90213609 & 1.53282172\end{array}$

9a , IEF-PCM (chloroform) /M06/6-311G(d), E=-1504.824943870307

$\begin{array}{lrrr}\mathrm{C} & -2.84269822 & -1.73276971 & -0.83087004 \\ \mathrm{C} & -2.70205741 & -2.68106651 & 0.18257248 \\ \mathrm{C} & -3.77647110 & -3.45078473 & 0.57183514 \\ \mathrm{C} & -5.01052040 & -3.25516740 & -0.05088218 \\ \mathrm{C} & -5.14325624 & -2.29937123 & -1.04739017 \\ \mathrm{C} & -4.06028583 & -1.51956133 & -1.45240833 \\ \mathrm{C} & -0.79722749 & -1.31411312 & 0.10528515 \\ \mathrm{C} & -1.26143407 & -2.70293457 & 0.63713255 \\ \mathrm{H} & -3.66885453 & -4.19623506 & 1.35919915 \\ \mathrm{H} & -5.87105974 & -3.84704628 & 0.25072645 \\ \mathrm{H} & -6.11107713 & -2.14547950 & -1.51940059 \\ \mathrm{H} & -4.18532686 & -0.76850969 & -2.22909014 \\ \mathrm{~N} & -1.59931962 & -1.12345577 & -1.08164603 \\ \mathrm{C} & -0.52657016 & -3.82993089 & -0.09035067 \\ \mathrm{H} & 0.53184033 & -3.86763304 & 0.19461451 \\ \mathrm{H} & -0.98086977 & -4.78907974 & 0.18120472 \\ \mathrm{H} & -0.59234492 & -3.72687116 & -1.17875039 \\ \mathrm{C} & -1.07312978 & -2.85852076 & 2.13486619 \\ \mathrm{H} & -1.34501736 & -3.87629388 & 2.43701584 \\ \mathrm{H} & -0.02357720 & -2.70458954 & 2.41591847 \\ \mathrm{H} & -1.68947782 & -2.15764704 & 2.70170659 \\ \mathrm{O} & -1.16939119 & -0.35367690 & 1.12962435 \\ \mathrm{C} & -1.51811563 & 0.20619279 & -1.65015187 \\ \mathrm{H} & -2.24372555 & 0.30297959 & -2.46393511 \\ \mathrm{H} & -0.52709127 & 0.33063740 & -2.10916780 \\ \mathrm{C} & -1.74196366 & 1.24227729 & -0.58983418 \\ \mathrm{C} & -1.53454352 & 0.88431979 & 0.74967147 \\ \mathrm{C} & -1.72232617 & 1.81764307 & 1.77192391 \\ \mathrm{H} & -1.55752212 & 1.50334432 & 2.79881723 \\ \mathrm{C} & -2.13927720 & 2.53399179 & -0.88082453 \\ \mathrm{H} & -2.31730851 & 2.83780975 & -1.90909643 \\ \mathrm{C} & -2.11218997 & 3.10368426 & 1.47259316 \\ \mathrm{C} & -2.31767037 & 3.45062989 & 0.14237870 \\ \mathrm{~N} & -2.74099006 & 4.80894019 & -0.18655754 \\ \mathrm{O} & -2.87626829 & 5.60063225 & 0.72688521 \\ \mathrm{O} & -2.93573312 & 5.07766377 & -1.35716649\end{array}$




$\begin{array}{rrrr}\mathrm{H} & -2.26202787 & 3.84253132 & 2.25220697 \\ \mathrm{C} & 0.66105386 & -1.18219322 & -0.19288135 \\ \mathrm{H} & 0.98006449 & -1.72279880 & -1.08384595 \\ \mathrm{C} & 1.52718048 & -0.48007975 & 0.53627792 \\ \mathrm{H} & 1.15807201 & 0.03474757 & 1.42451840 \\ \mathrm{C} & 2.95594499 & -0.32617281 & 0.26920646 \\ \mathrm{C} & 3.56946646 & -0.80115255 & -0.89732445 \\ \mathrm{C} & 3.74436759 & 0.33843295 & 1.21472765 \\ \mathrm{C} & 4.92027669 & -0.62270766 & -1.11125518 \\ \mathrm{H} & 2.98375016 & -1.31086107 & -1.65771299 \\ \mathrm{C} & 5.09977399 & 0.52328452 & 1.01637639 \\ \mathrm{H} & 3.28023011 & 0.71660549 & 2.12286019 \\ \mathrm{C} & 5.67032015 & 0.03872863 & -0.14843132 \\ \mathrm{H} & 5.40078327 & -0.98343209 & -2.01420813 \\ \mathrm{H} & 5.71480044 & 1.03799168 & 1.74656088 \\ \mathrm{O} & 7.58281864 & -0.19998411 & -1.40465212 \\ \mathrm{~N} & 7.10785726 & 0.23453115 & -0.37447535 \\ \mathrm{O} & 7.74120211 & 0.81958602 & 0.48095322\end{array}$

$10 \mathrm{a}, \mathrm{IEF}-\mathrm{PCM}$ (chloroform) /M06/6-311G(d), E=-1413.656373187413

$\begin{array}{lrrr}\mathrm{C} & -2.49406256 & -1.80667927 & -0.84646568 \\ \mathrm{C} & -2.31699437 & -2.75157390 & 0.16454413 \\ \mathrm{C} & -3.36059847 & -3.56318054 & 0.55209774 \\ \mathrm{C} & -4.60161856 & -3.41373414 & -0.06987926 \\ \mathrm{C} & -4.77143450 & -2.46067063 & -1.06325244 \\ \mathrm{C} & -3.71953142 & -1.63814636 & -1.46630173 \\ \mathrm{C} & -0.47107502 & -1.30535596 & 0.09803219 \\ \mathrm{C} & -0.87783554 & -2.71512814 & 0.62257732 \\ \mathrm{H} & -3.22430135 & -4.30506491 & 1.33852337 \\ \mathrm{H} & -5.43882400 & -4.03884647 & 0.23039602 \\ \mathrm{H} & -5.74487183 & -2.34207603 & -1.53397603 \\ \mathrm{H} & -3.87365921 & -0.88793271 & -2.23873956 \\ \mathrm{~N} & -1.27641347 & -1.14583342 & -1.09144307 \\ \mathrm{C} & -0.09466838 & -3.80692604 & -0.10800616 \\ \mathrm{H} & 0.96359509 & -3.80066521 & 0.17942811 \\ \mathrm{H} & -0.50900652 & -4.78554096 & 0.15822748 \\ \mathrm{H} & -0.16199213 & -3.70178843 & -1.19608603 \\ \mathrm{C} & -0.68692566 & -2.86962668 & 2.11979256 \\ \mathrm{H} & -0.91833992 & -3.89881920 & 2.41703369 \\ \mathrm{H} & 0.35508746 & -2.67459575 & 2.40325711 \\ \mathrm{H} & -1.33217845 & -2.19626237 & 2.68782721 \\ \mathrm{O} & -0.89271869 & -0.36595596 & 1.12478758 \\ \mathrm{C} & -1.24951923 & 0.18792065 & -1.65520332 \\ \mathrm{H} & -1.97104867 & 0.25528759 & -2.47573799 \\ \mathrm{H} & -0.26047897 & 0.35694156 & -2.10412781 \\ \mathrm{C} & -1.52954227 & 1.20755018 & -0.59257220 \\ \mathrm{C} & -1.31807082 & 0.85258352 & 0.74728541 \\ \mathrm{C} & -1.56354885 & 1.77025675 & 1.77197517 \\ \mathrm{H} & -1.39557484 & 1.45862130 & 2.79917948 \\ \mathrm{C} & -1.98371729 & 2.48060499 & -0.88177960 \\ \mathrm{H} & -2.16458918 & 2.78057425 & -1.91067823 \\ \mathrm{C} & -2.00996846 & 3.03802246 & 1.47445867 \\ \mathrm{C} & -2.21567205 & 3.38286977 & 0.14367535 \\ \mathrm{~N} & -2.69591289 & 4.72234395 & -0.18286849 \\ \mathrm{O} & -2.87275904 & 5.50341196 & 0.73280703 \\ \mathrm{O} & -2.89317028 & 4.98797090 & -1.35386214 \\ \mathrm{H} & -2.20314386 & 3.76471560 & 2.25594925 \\ \mathrm{C} & 0.98109297 & -1.10749469 & -0.19057393 \\ \mathrm{H} & 1.32647961 & -1.62884834 & -1.08312717 \\ \mathrm{C} & 1.81325592 & -0.37613506 & 0.54987037 \\ \mathrm{H} & 1.41411895 & 0.11820651 & 1.43706437 \\ \mathrm{C} & 3.23794453 & -0.16355215 & 0.30168300 \\ \mathrm{C} & 3.89160429 & -0.63564306 & -0.84738060 \\ \mathrm{C} & 3.98059134 & 0.54950192 & 1.24721286 \\ \mathrm{H} & 5.23567271 & -0.40446587 & -1.03657737 \\ & 3.33686399 & -1.18445607 & -1.60465805\end{array}$




$\begin{array}{lrrr}\mathrm{C} & 5.33230528 & 0.77989308 & 1.05954443 \\ \mathrm{H} & 3.48302078 & 0.92438932 & 2.13967369 \\ \mathrm{C} & 5.96928579 & 0.30550722 & -0.08226073 \\ \mathrm{H} & 5.74647735 & -0.76539027 & -1.92619537 \\ \mathrm{H} & 5.90301299 & 1.33510291 & 1.80289385 \\ \mathrm{C} & 7.40438910 & 0.55418203 & -0.27755481 \\ \mathrm{O} & 8.04623027 & 0.18824339 & -1.23061727 \\ \mathrm{H} & 7.88682771 & 1.12803112 & 0.54948364\end{array}$

$11 \mathrm{a}, \mathrm{IEF}-\mathrm{PCM}$ (chloroform) /M06/6-311G(d), $E=-1777.54812811057$

\begin{tabular}{|c|c|c|c|}
\hline C & 4.62166418 & -1.51650440 & 0.68332173 \\
\hline C & 4.53910121 & -2.42429604 & -0.37376285 \\
\hline C & 5.67343076 & -3.04061380 & -0.85408566 \\
\hline C & 6.90834530 & -2.73313292 & -0.27877089 \\
\hline C & 6.98138924 & -1.82065328 & 0.76312870 \\
\hline C & 5.83799068 & -1.19470553 & 1.25980050 \\
\hline C & 2.49648801 & -1.29090749 & -0.14049694 \\
\hline C & 3.08842878 & -2.59084998 & -0.76000370 \\
\hline $\mathrm{H}$ & 5.61158881 & -3.75206552 & -1.67706834 \\
\hline $\mathrm{H}$ & 7.81456192 & -3.20307674 & -0.65297874 \\
\hline $\mathrm{H}$ & 7.94790786 & -1.57871838 & 1. 19981053 \\
\hline $\mathrm{H}$ & 5.91594565 & -0.47397581 & 2.07065319 \\
\hline $\mathrm{N}$ & 3.33334869 & -1.06642158 & 1.01869160 \\
\hline C & 2.52506201 & -3.82295149 & -0.04895898 \\
\hline $\mathrm{H}$ & 1.46488342 & -3.97211043 & -0.28563589 \\
\hline $\mathrm{H}$ & 3.07336679 & -4.71192614 & -0.38029185 \\
\hline $\mathrm{H}$ & 2.62926096 & -3.75135152 & 1.03890259 \\
\hline C & 2.83737988 & -2.70888893 & -2.25186211 \\
\hline $\mathrm{H}$ & 3.20078374 & -3.67794355 & -2.61321097 \\
\hline $\mathrm{H}$ & 1.76305631 & -2.66132295 & -2.47007003 \\
\hline $\mathrm{H}$ & 3.34085568 & -1.92329324 & -2.81950189 \\
\hline 0 & 2.72615493 & -0.24385690 & -1.13049755 \\
\hline C & 3. 12958777 & $\odot .21550392$ & 1.65882234 \\
\hline $\mathrm{H}$ & 3.86369848 & $\odot .35241886$ & 2.45930903 \\
\hline $\mathrm{H}$ & 2.14427115 & $\odot .20702192$ & 2.14458916 \\
\hline C & 3.20796273 & 1.31758048 & 0.64599674 \\
\hline C & 2.97613282 & 1.00346960 & -0.70182072 \\
\hline C & 3.02538781 & 2. 00169321 & -1.67948034 \\
\hline $\mathrm{H}$ & 2.84381188 & 1.72378632 & -2.71410693 \\
\hline C & 3.49784062 & 2.62488497 & 0.98691661 \\
\hline $\mathrm{H}$ & 3.69536254 & 2.89212586 & 2.02171620 \\
\hline C & 3.30857770 & 3.30273670 & -1.32978847 \\
\hline C & 3.54487960 & 3.60383958 & $\odot .00675500$ \\
\hline $\mathrm{N}$ & 3.85925348 & 4.97688104 & $\odot .38683085$ \\
\hline 0 & 3.89943846 & 5.81796673 & -0.49184681 \\
\hline 0 & 4.06582640 & 5. 21089695 & 1.56333138 \\
\hline $\mathrm{H}$ & 3.35335268 & 4.08783839 & -2.07648418 \\
\hline C & 1.04904459 & -1.33601347 & ๑. 21989591 \\
\hline $\mathrm{H}$ & $\odot .80541068$ & -1.95010323 & 1.08235457 \\
\hline C & $\odot .08950696$ & -0.68929073 & -0.44789082 \\
\hline $\mathrm{H}$ & $\odot .38665185$ & -0.08495997 & -1.30790649 \\
\hline C & -1.33844647 & -0.67124174 & -0.18200693 \\
\hline C & -2.17012914 & $\odot .06217798$ & -0.97933961 \\
\hline C & -1.90866225 & -1.41657844 & 0.93299263 \\
\hline C & -3.56446560 & $\odot .13148295$ & -0.76367970 \\
\hline $\mathrm{H}$ & -1.74463531 & $\odot .62137176$ & -1.81395029 \\
\hline C & -4.47016924 & $\odot .86990266$ & -1.54306139 \\
\hline C & -4.09424339 & -0.58637077 & ๑. 31614388 \\
\hline C & -5.80988170 & $\odot .88695665$ & -1.26200750 \\
\hline $\mathrm{H}$ & -4.09141938 & 1.43410444 & -2.39353906 \\
\hline C & -5.43728484 & $-\odot .58162877$ & 0.62525375 \\
\hline C & -6.34075349 & $\odot .15714994$ & -0.16161294 \\
\hline $\mathrm{H}$ & -6.46887947 & 1.45858142 & -1.90611825 \\
\hline $\mathrm{H}$ & -5.75300475 & -1.14772655 & 1.49483041 \\
\hline 0 & -1.31763948 & -2.11009017 & 1. 71841232 \\
\hline 0 & -3.27519671 & -1.31713648 & 1. 11232456 \\
\hline
\end{tabular}




$\begin{array}{rrrr}\mathrm{N} & -7.67453396 & 0.17178693 & 0.11626895 \\ \mathrm{C} & -8.24739076 & -0.65861574 & 1.16571154 \\ \mathrm{H} & -9.28337132 & -0.87814465 & 0.87961165 \\ \mathrm{H} & -7.73979493 & -1.62976836 & 1.18327853 \\ \mathrm{C} & -8.60918061 & 1.01895760 & -0.61094676 \\ \mathrm{H} & -9.42811329 & 1.26364150 & 0.07675897 \\ \mathrm{H} & -8.13355177 & 1.97796322 & -0.84401767 \\ \mathrm{C} & -9.15686738 & 0.35787986 & -1.86106127 \\ \mathrm{H} & -9.87670410 & 1.01027760 & -2.36616506 \\ \mathrm{H} & -8.35822572 & 0.12021295 & -2.57200699 \\ \mathrm{H} & -9.66877320 & -0.57920477 & -1.61461336 \\ \mathrm{C} & -8.21001976 & 0.00448700 & 2.52938300 \\ \mathrm{H} & -8.66456258 & -0.63854475 & 3.29009270 \\ \mathrm{H} & -7.18277718 & 0.22200691 & 2.84071088 \\ \mathrm{H} & -8.76034452 & 0.95213862 & 2.51781001\end{array}$

$12 \mathrm{a}, \operatorname{IEF}-\mathrm{PCM}$ (chloroform) /M06/6-311G(d), $E=-1624.717617375786$

\begin{tabular}{|c|c|c|c|}
\hline & & & \\
\hline$c$ & -3.41802765 & -2.09179611 & -0.74627575 \\
\hline C & -3.21021474 & -2.90570980 & ๑. 36829381 \\
\hline C & -4.16877237 & -3.81541143 & $\odot .75730666$ \\
\hline C & -5.35774991 & -3.90071838 & $\odot .02991495$ \\
\hline C & -5.55995680 & -3.07940970 & -1.06892262 \\
\hline C & -4.59324318 & -2.15896407 & -1.47421246 \\
\hline C & -1.56939636 & -1.22918247 & ๑. 29316684 \\
\hline C & -1.83517027 & -2.62747592 & ๑. 92684959 \\
\hline $\mathrm{H}$ & -4.00680244 & -4.45351318 & 1.62534732 \\
\hline $\mathrm{H}$ & -6.12899263 & -4.60599009 & 0. 32946283 \\
\hline $\mathrm{H}$ & -6.49254744 & -3.14478566 & -1.62508240 \\
\hline $\mathrm{H}$ & -4.77337910 & -1.51752032 & -2.33370612 \\
\hline $\mathrm{N}$ & -2.28257608 & -1.29300944 & -0.96386866 \\
\hline C & -0.85359968 & -3.65987953 & 0.36987781 \\
\hline $\mathrm{H}$ & 0.16555793 & -3.47666861 & $\odot .73133267$ \\
\hline H & -1.15393257 & -4.65994413 & 0.70185468 \\
\hline $\mathrm{H}$ & -0.83904268 & -3.66180053 & -0.72537275 \\
\hline C & -1.75649231 & -2.62070593 & 2.44201399 \\
\hline $\mathrm{H}$ & -1.89088927 & -3.63940252 & 2.82391884 \\
\hline $\mathrm{H}$ & -0.77235469 & -2.27191607 & 2.77927486 \\
\hline $\mathrm{H}$ & -2.52178200 & -1.98413717 & 2.89132082 \\
\hline 0 & -2.21377510 & $-\odot .27184985$ & 1.17981749 \\
\hline C & -2.37418403 & -0.03073394 & -1.66688738 \\
\hline $\mathrm{H}$ & -3.05533576 & $-\odot .13507189$ & -2.51758705 \\
\hline $\mathrm{H}$ & -1.38928341 & ๑. 20945395 & -2.09127822 \\
\hline C & -2.82369084 & 1.05854864 & -0.73853475 \\
\hline C & -2.69841289 & 0.86061372 & 0.64413470 \\
\hline C & -3.09960572 & 1.85328732 & 1.54257935 \\
\hline $\mathrm{H}$ & -2.99487677 & 1.66189940 & 2.60706393 \\
\hline C & -3.34111162 & 2.25602917 & -1.19582857 \\
\hline $\mathrm{H}$ & -3.45193758 & 2.43636210 & -2.26205215 \\
\hline C & -3.61128550 & 3.04381586 & 1.07833353 \\
\hline C & -3.72429946 & 3.23587979 & -0.29407393 \\
\hline N & -4.26220423 & 4.49501930 & -0.79791573 \\
\hline 0 & -4.58151109 & 5.34400898 & ๑.01317095 \\
\hline 0 & -4.36192819 & 4.63257025 & -2.00321925 \\
\hline $\mathrm{H}$ & -3.92316837 & 3.82734556 & 1.76030526 \\
\hline C & -0.13509192 & -0.85738864 & ๑. 11402291 \\
\hline $\mathrm{H}$ & $\odot .36148987$ & -1.38422944 & -0.70162777 \\
\hline C & 0.52824983 & 0.01176801 & 0.87777961 \\
\hline $\mathrm{H}$ & -0.02916395 & $\odot .53305981$ & 1.65892331 \\
\hline C & 1.94412027 & $\odot .35768285$ & $\odot .78812365$ \\
\hline C & 2.40442211 & 1.48328741 & 1.49685637 \\
\hline C & 2.85990190 & $-\odot .38998186$ & $\odot .04750694$ \\
\hline C & 3.72523459 & 1.88963523 & 1.47327638 \\
\hline $\mathrm{H}$ & 1.68740440 & 2.05527219 & 2.08391114 \\
\hline C & 4. 19177738 & $-\odot .0 \odot 782003$ & 0.00839996 \\
\hline $\mathrm{H}$ & 2.54043302 & -1.28582751 & -0.48370685 \\
\hline C & 4.62130923 & 1.13847852 & $\odot .71979467$ \\
\hline
\end{tabular}




$\begin{array}{lrrr}\mathrm{H} & 4.03999348 & 2.76299088 & 2.03886815 \\ \mathrm{C} & 5.36264490 & -0.55815983 & -0.62756179 \\ \mathrm{C} & 6.44036450 & 0.28092783 & -0.27392893 \\ \mathrm{C} & 5.59160179 & -1.66371840 & -1.44109947 \\ \mathrm{C} & 7.73663897 & 0.03013891 & -0.71066476 \\ \mathrm{C} & 6.87976027 & -1.91781331 & -1.88197130 \\ \mathrm{H} & 4.77131412 & -2.32101513 & -1.72453236 \\ \mathrm{C} & 7.93765087 & -1.07871166 & -1.51782244 \\ \mathrm{H} & 8.56714565 & 0.67330665 & -0.42869587 \\ \mathrm{H} & 7.07455022 & -2.77930897 & -2.51593105 \\ \mathrm{H} & 8.94122547 & -1.30075958 & -1.87350330 \\ \mathrm{~N} & 5.97664871 & 1.30466343 & 0.53064875 \\ \mathrm{C} & 6.81055339 & 2.33324402 & 1.09597353 \\ \mathrm{H} & 7.39428516 & 2.82779281 & 0.31220884 \\ \mathrm{H} & 6.18917466 & 3.08830572 & 1.57906034 \\ \mathrm{H} & 7.50200210 & 1.92301617 & 1.84149223\end{array}$

Photochemically open (b)

\begin{tabular}{|c|c|c|c|}
\hline C & -3.69723629 & -1.04625676 & -0.01610528 \\
\hline C & -3.77649031 & ๑. 29912096 & -0.33914761 \\
\hline C & -4.98410291 & ๑.95481430 & -0.50079874 \\
\hline C & -6.14004787 & 0.20232877 & -0.32935185 \\
\hline C & -6.07857709 & -1.15074592 & -0.01174956 \\
\hline C & -4.85065679 & -1.78695584 & 0.14910520 \\
\hline C & -2.25029666 & -1.43954615 & ๑. 11102366 \\
\hline C & -1.55665493 & -0.12280559 & -0.20237616 \\
\hline $\mathrm{H}$ & -5.03916383 & 2.01271792 & -0.73936511 \\
\hline $\mathrm{H}$ & -7.10782111 & 0.68366293 & -0.44416819 \\
\hline $\mathrm{H}$ & -6.99914832 & -1.71424446 & 0.11659779 \\
\hline $\mathrm{H}$ & -4.80564162 & -2.84429986 & $\odot .4 \odot 499061$ \\
\hline $\mathrm{N}$ & -2.46806347 & 0.81824653 & -0.47214291 \\
\hline C & -0.17228363 & ๑. 14230143 & -0.24819152 \\
\hline $\mathrm{H}$ & $\odot .10685843$ & 1.16385150 & -0.49516376 \\
\hline C & $\odot .82301426$ & -0.75522061 & $\odot .00 \odot 54928$ \\
\hline $\mathrm{H}$ & $\odot .55753732$ & -1.77938820 & $\odot .26050895$ \\
\hline C & 2.23042191 & -0.49961600 & -0.02975742 \\
\hline C & 3. 12813226 & -1.54844250 & $\odot .23628171$ \\
\hline C & 2.79396666 & 0.76028739 & -0.30796568 \\
\hline C & 4.49031272 & -1.36938861 & $\odot .22777581$ \\
\hline $\mathrm{H}$ & 2.72917720 & -2.53802016 & $\odot .45636593$ \\
\hline C & 4.15076153 & $\odot .95895274$ & -0.31900928 \\
\hline $\mathrm{H}$ & 2.15123429 & 1.61237422 & -0.51705071 \\
\hline C & 5.04988607 & -0.10158640 & -0.04898917 \\
\hline $\mathrm{H}$ & 5.13233565 & -2.21771068 & $\odot .44060835$ \\
\hline $\mathrm{H}$ & 4.52826812 & 1.95247407 & -0.53635755 \\
\hline $\mathrm{N}$ & 6.39594268 & ๑.09419139 & -0.05422485 \\
\hline C & 7. 29116930 & $-1.0 \odot 984761$ & $\odot .21446346$ \\
\hline $\mathrm{H}$ & 7.18279480 & -1.81244567 & -0.52705882 \\
\hline $\mathrm{H}$ & 8.32157408 & $-\odot .65690607$ & $\odot .17678600$ \\
\hline $\mathrm{H}$ & 7.11996276 & -1.44021409 & 1.20968117 \\
\hline C & 6.94377137 & 1.40215957 & -0.34389354 \\
\hline $\mathrm{H}$ & 6.60700620 & 2.15383287 & 0.38139039 \\
\hline $\mathrm{H}$ & 8.03152915 & 1.35588460 & -0.29565900 \\
\hline $\mathrm{H}$ & 6.66567346 & 1.74827445 & -1.34786365 \\
\hline C & -2.21611208 & 2.23482093 & -0.54431827 \\
\hline $\mathrm{H}$ & -1.30941509 & 2.44169874 & -1.11725339 \\
\hline $\mathrm{H}$ & -3.05197493 & 2.72809575 & -1.04453787 \\
\hline C & -2.04124547 & 2.86281947 & $\odot .90497191$ \\
\hline $\mathrm{H}$ & -1.21774184 & 2.20588777 & 1.33965834 \\
\hline $\mathrm{H}$ & -2.97338368 & 2.52021777 & 1.45867007 \\
\hline 0 & -1.81624025 & 4.13638621 & 0.84530354 \\
\hline C & -1.90835603 & -2.53228519 & -0.90393182 \\
\hline $\mathrm{H}$ & $-\odot .85850796$ & -2.83417215 & -0.85281430 \\
\hline $\mathrm{H}$ & -2.52004115 & -3.41972542 & -0.70721909 \\
\hline $\mathrm{H}$ & -2.11700826 & -2.19857991 & -1.92611673 \\
\hline
\end{tabular}




\begin{tabular}{|c|c|c|c|}
\hline $\begin{array}{l}\mathrm{C} \\
\mathrm{H} \\
\mathrm{H} \\
\mathrm{H}\end{array}$ & $\begin{array}{l}-1.96293184 \\
-0.91632382 \\
-2.19907677 \\
-2.58260106\end{array}$ & $\begin{array}{l}-1.88647252 \\
-2.16969264 \\
-1.08660809 \\
-2.75573533\end{array}$ & $\begin{array}{l}1.54820683 \\
1.69188274 \\
2.25896503 \\
1.79591720\end{array}$ \\
\hline $\begin{array}{l}\mathrm{C} \\
\mathrm{C} \\
\mathrm{C} \\
\mathrm{C} \\
\mathrm{C} \\
\mathrm{H} \\
\mathrm{H} \\
\mathrm{H} \\
\mathrm{H} \\
\mathrm{C} \\
\mathrm{C} \\
\mathrm{C} \\
\mathrm{C} \\
\mathrm{C} \\
\mathrm{C} \\
\mathrm{H} \\
\mathrm{C} \\
\mathrm{C} \\
\mathrm{C} \\
\mathrm{H} \\
\mathrm{H} \\
\mathrm{S} \\
\mathrm{N} \\
0 \\
\mathrm{C} \\
\mathrm{H} \\
\mathrm{H} \\
\mathrm{H} \\
\mathrm{C} \\
\mathrm{H} \\
\mathrm{H} \\
\mathrm{H} \\
\mathrm{N} \\
0 \\
\mathrm{C} \\
\mathrm{H} \\
\mathrm{H} \\
\mathrm{H} \\
0 \\
0\end{array}$ & $\begin{array}{r}\text { IEF-PCM (chlor of } \\
4.52534794 \\
4.03531056 \\
4.88122098 \\
6.21562181 \\
6.70487976 \\
5.86340784 \\
4.50843557 \\
6.88995354 \\
7.75613417 \\
6.23716953 \\
2.07798395 \\
0.70280499 \\
-2.18594965 \\
-0.21744164 \\
-3.65204641 \\
-1.62550846 \\
0.18172596 \\
-4.43672803 \\
-2.48155282 \\
-3.83801290 \\
-5.51818293 \\
-2.10167681 \\
3.24060650 \\
2.67512706 \\
-1.48998445 \\
0.32998088 \\
1.19034758 \\
-0.37443887 \\
-0.14553465 \\
2.06525752 \\
2.80872685 \\
1.71987530 \\
1.21994099 \\
-4.69119167 \\
-4.09947780 \\
-5.49162909 \\
-5.91869541 \\
-5.99335138 \\
-5.65798599 \\
-4.18066753 \\
-5.90204253\end{array}$ & $\begin{array}{r}r m) / M 06 / 6-31 \\
-0.62197354 \\
0.58723574 \\
1.68064230 \\
1.52457444 \\
0.30900708 \\
-0.77938562 \\
2.63769703 \\
2.36974172 \\
0.21445624 \\
-1.72757780 \\
-0.64266400 \\
-0.97406234 \\
1.42471278 \\
0.04071759 \\
1.50984480 \\
0.06887872 \\
1.03757334 \\
0.40438269 \\
-1.04473810 \\
-0.87704203 \\
0.45794509 \\
-2.05361631 \\
-1.80039992 \\
0.53172708 \\
2.43822703 \\
-2.42250295 \\
-3.05945832 \\
-2.56974372 \\
-2.81220751 \\
1.60278717 \\
1.98038787 \\
2.42176493 \\
1.20714688 \\
-2.03341157 \\
2.77264778 \\
2.95635511 \\
2.43649148 \\
2.59867293 \\
4.02868632 \\
-3.14004872 \\
-1.86212474\end{array}$ & 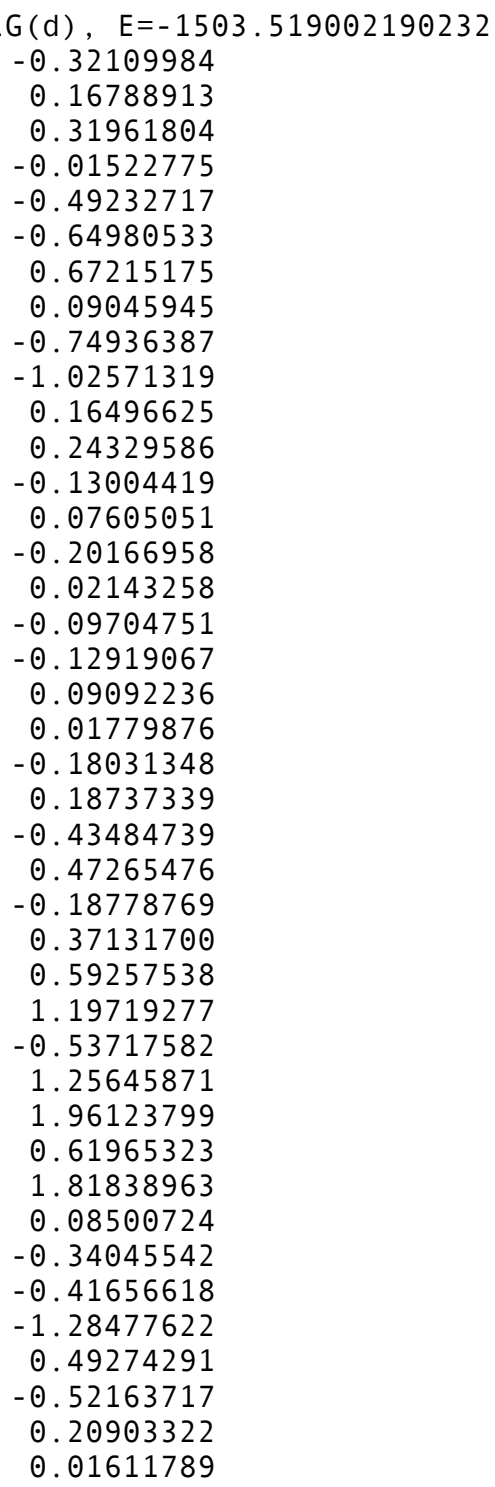 \\
\hline $\begin{array}{l}\text { C } \\
\text { C } \\
\text { C } \\
\text { C } \\
\text { C } \\
\text { C } \\
\text { C } \\
\text { H } \\
\text { H } \\
\text { H } \\
\text { H } \\
\text { N } \\
\text { C } \\
\text { H } \\
\text { H } \\
\text { H } \\
\text { C }\end{array}$ & $\begin{array}{c}\text { EF-PCM (chlor of } \\
-1.83264828 \\
-1.35031147 \\
-2.18214514 \\
-3.50190862 \\
-3.97160944 \\
-3.14099976 \\
0.32939987 \\
0.09337896 \\
-1.82075328 \\
-4.17483735 \\
-5.00699203 \\
-3.52233461 \\
-0.78474069 \\
0.26254390 \\
1.30181149 \\
-0.04910598 \\
-0.35517386 \\
0.98385461\end{array}$ & $\begin{array}{l}\text { m) } / \mathrm{M} 06 / 6-31 \\
1.96215723 \\
2.94360784 \\
3.95962244 \\
3.96691243 \\
2.97132183 \\
1.94476723 \\
1.39222345 \\
2.65829395 \\
4.73736813 \\
4.75598112 \\
2.98896282 \\
1.16937954 \\
1.05659558 \\
2.40127705 \\
2.18629614 \\
3.28944870 \\
1.55923639 \\
3.80664850\end{array}$ & $\begin{array}{l}G(d), E=-1621.143988332227 \\
-0.67890173 \\
0.17555293 \\
0.59942076 \\
0.15578394 \\
-0.69370873 \\
-1.12849055 \\
-0.31354688 \\
0.49137881 \\
1.26986850 \\
0.48052781 \\
-1.02239460 \\
-1.78650130 \\
-0.95837162 \\
1.99207722 \\
2.25659811 \\
2.55209999 \\
2.32162608 \\
0.00931200\end{array}$ \\
\hline
\end{tabular}




$\begin{array}{rrrr}\mathrm{H} & 0.69569372 & 4.73042813 & 0.52223779 \\ \mathrm{H} & 2.04148719 & 3.62579057 & 0.22081491 \\ \mathrm{H} & 0.87297766 & 3.97024644 & -1.06772158 \\ \mathrm{O} & 1.01234360 & -2.16965414 & -1.71542732 \\ \mathrm{C} & -0.95987262 & -0.13557895 & -1.81407728 \\ \mathrm{H} & -0.08509697 & -0.21957091 & -2.46417303 \\ \mathrm{H} & -1.81904728 & 0.07741997 & -2.45522103 \\ \mathrm{C} & -1.12989055 & -1.40612645 & -1.04003959 \\ \mathrm{C} & -0.05302398 & -2.37741806 & -1.08298957 \\ \mathrm{C} & -0.28730189 & -3.58859358 & -0.34346786 \\ \mathrm{H} & 0.50563980 & -4.33378229 & -0.36263886 \\ \mathrm{C} & -2.28486710 & -1.65062435 & -0.33767187 \\ \mathrm{H} & -3.09345178 & -0.92192264 & -0.31864287 \\ \mathrm{C} & -1.43798243 & -3.81350350 & 0.35515786 \\ \mathrm{C} & -2.45213603 & -2.84225788 & 0.36918785 \\ \mathrm{~N} & -3.65922256 & -3.07435579 & 1.09430157 \\ \mathrm{O} & -3.78366151 & -4.13116637 & 1.70557633 \\ \mathrm{O} & -4.53169922 & -2.20963813 & 1.08329157 \\ \mathrm{H} & -1.58865937 & -4.73635613 & 0.90759264 \\ \mathrm{C} & 1.49078741 & 0.60264376 & -0.41135084 \\ \mathrm{H} & 1.41930144 & -0.29990388 & -1.02118760 \\ \mathrm{C} & 2.67621194 & 0.85003666 & 0.21332792 \\ \mathrm{H} & 2.80749089 & 1.74646831 & 0.81832768 \\ \mathrm{C} & 3.79654850 & -0.02111099 & 0.12552695 \\ \mathrm{C} & 3.88732747 & -1.26173550 & -0.48189881 \\ \mathrm{C} & 6.03394360 & -1.04017359 & 0.34437686 \\ \mathrm{H} & 3.04238680 & -1.73945531 & -0.97989161 \\ \mathrm{C} & 5.16129997 & -1.83396928 & -0.35406186 \\ \mathrm{H} & 7.07136722 & -1.24197951 & 0.58083777 \\ \mathrm{H} & 5.43596586 & -2.80260490 & -0.75764470 \\ \mathrm{~S} & 5.31685188 & 0.42100083 & 0.86631766\end{array}$

4b, IEF-PCM (chloroform) /M06/6-311G(d), E=-1621.143449971587

$\begin{array}{lrrr}\mathrm{C} & 2.04919919 & -1.81507928 & -0.66131974 \\ \mathrm{C} & 1.70589933 & -2.85530887 & 0.19020993 \\ \mathrm{C} & 2.65963895 & -3.77403851 & 0.57811977 \\ \mathrm{C} & 3.95846844 & -3.62537857 & 0.09901996 \\ \mathrm{C} & 4.28741831 & -2.57278899 & -0.74822970 \\ \mathrm{C} & 3.33332869 & -1.64333935 & -1.14674955 \\ \mathrm{C} & -0.15573994 & -1.51066941 & -0.24403990 \\ \mathrm{C} & 0.24571990 & -2.74817891 & 0.53791979 \\ \mathrm{H} & 2.40715905 & -4.59742819 & 1.24372951 \\ \mathrm{H} & 4.72561814 & -4.33655829 & 0.39310984 \\ \mathrm{H} & 5.30798790 & -2.46963903 & -1.10648956 \\ \mathrm{H} & 3.60373858 & -0.82341967 & -1.80595929 \\ \mathrm{~N} & 0.89409965 & -1.03670959 & -0.90562964 \\ \mathrm{C} & 0.07531997 & -2.53727900 & 2.04557919 \\ \mathrm{H} & -0.97619961 & -2.44700903 & 2.33260908 \\ \mathrm{H} & 0.49624980 & -3.39426866 & 2.58223898 \\ \mathrm{H} & 0.60041976 & -1.63709935 & 2.38153906 \\ \mathrm{C} & -0.51140980 & -3.98655843 & 0.04959998 \\ \mathrm{H} & -0.11145996 & -4.87630808 & 0.54748978 \\ \mathrm{H} & -1.58051938 & -3.93020845 & 0.27349989 \\ \mathrm{H} & -0.39442984 & -4.12285837 & -1.03068959 \\ \mathrm{O} & -1.31372948 & 1.93861924 & -1.58841937 \\ \mathrm{C} & 0.90351965 & 0.16653993 & -1.76589930 \\ \mathrm{H} & 0.01200000 & 0.13669995 & -2.39797905 \\ \mathrm{H} & 1.76947930 & 0.05613998 & -2.42333905 \\ \mathrm{C} & 0.93591963 & 1.45483943 & -1.00233960 \\ \mathrm{C} & -0.25573990 & 2.28309910 & -1.00397960 \\ \mathrm{C} & -0.14298994 & 3.52367861 & -0.28542989 \\ \mathrm{H} & -1.02328959 & 4.16345836 & -0.27520989 \\ \mathrm{C} & 2.08073918 & 1.85455927 & -0.35620986 \\ \mathrm{H} & 2.97637883 & 1.23622951 & -0.36921985 \\ \mathrm{C} & 0.99953961 & 3.90155846 & 0.35806986 \\ \mathrm{C} & 2.12773916 & 3.06625879 & 0.33336987\end{array}$




$\begin{array}{rrrr}\mathrm{N} & 3.32765869 & 3.45814864 & 0.99979961 \\ \mathrm{O} & 3.34429868 & 4.52913822 & 1.59804937 \\ \mathrm{O} & 4.30133830 & 2.71104893 & 0.95271962 \\ \mathrm{H} & 1.05650959 & 4.84445809 & 0.89434965 \\ \mathrm{C} & -1.40972944 & -0.86944966 & -0.31152988 \\ \mathrm{H} & -1.46249942 & 0.03935998 & -0.91411964 \\ \mathrm{C} & -2.53983900 & -1.26945950 & 0.32759987 \\ \mathrm{H} & -2.54574900 & -2.18074914 & 0.92555964 \\ \mathrm{C} & -3.78312851 & -0.55736978 & 0.28064989 \\ \mathrm{C} & -3.96652844 & 0.67860973 & -0.31271988 \\ \mathrm{C} & -6.03679761 & -0.20620992 & 0.71102972 \\ \mathrm{H} & -3.22129873 & 1.30094949 & -0.80819968 \\ \mathrm{H} & -7.05943724 & -0.32206987 & 1.04581959 \\ \mathrm{~S} & -5.57182783 & 1.22078952 & -0.15616994 \\ \mathrm{C} & -4.99078803 & -1.04967959 & 0.87125966 \\ \mathrm{H} & -5.05746801 & -2.00294921 & 1.38801945\end{array}$

5b, IEF-PCM (chloroform) /M06/6-311G(d), E=-1817.507016782477

\begin{tabular}{|c|c|c|c|}
\hline C & -5.03090469 & -1.31354671 & -0.89950945 \\
\hline C & -4.84646298 & -2.54272837 & -0.28236198 \\
\hline C & -5.93250471 & -3.34658715 & -0.00223989 \\
\hline C & -7.20290194 & -2.89474600 & -0.35099220 \\
\hline C & -7.37128681 & -1.65934940 & -0.96587274 \\
\hline C & -6.28331906 & -0.84231340 & -1.25297083 \\
\hline C & -2.78804298 & -1.47052636 & -0.59331836 \\
\hline C & -3.37774132 & -2.75173466 & -0.02483051 \\
\hline $\mathrm{H}$ & -5.80463829 & -4.31383869 & ๑.48090992 \\
\hline $\mathrm{H}$ & -8.07200912 & -3.51092605 & -0.13691835 \\
\hline $\mathrm{H}$ & -8.37026106 & -1.31984449 & -1.22605287 \\
\hline $\mathrm{H}$ & -6.43087424 & $\odot .12263475$ & -1.72941785 \\
\hline $\mathrm{N}$ & -3.76934714 & -0.70511078 & -1.07567314 \\
\hline C & -2.88058269 & -3.98846397 & -0.77774675 \\
\hline $\mathrm{H}$ & -1.81343417 & -4.16875994 & -0.62150564 \\
\hline $\mathrm{H}$ & -3.42274638 & -4.87281542 & -0.42585357 \\
\hline $\mathrm{H}$ & -3.05610463 & -3.89245261 & -1.85452030 \\
\hline C & -3.12244802 & -2.85861410 & 1.48165121 \\
\hline $\mathrm{H}$ & -3.67089467 & -3.71657388 & 1.88507777 \\
\hline $\mathrm{H}$ & -2.06268336 & -2.99977148 & 1.71179140 \\
\hline $\mathrm{H}$ & -3.46801613 & -1.96010912 & 2.00393721 \\
\hline 0 & -1.10859931 & 1.96445094 & -1.44351532 \\
\hline C & -3.59784994 & 0.63292636 & -1.67295110 \\
\hline $\mathrm{H}$ & -4.50240717 & $\odot .82365878$ & -2.25624195 \\
\hline $\mathrm{H}$ & -2.76373185 & $\odot .58702704$ & -2.37873851 \\
\hline C & -3.33667530 & $1.7138540 \odot$ & -0.66846532 \\
\hline C & -2.02326577 & 2.33168445 & -0.66642137 \\
\hline C & -1.83995139 & 3.38506853 & 0.29610323 \\
\hline $\mathrm{H}$ & -0.86370370 & 3.86610543 & $\odot .31378515$ \\
\hline C & -4.31900914 & 2.12439984 & ๑. 19879827 \\
\hline $\mathrm{H}$ & -5.30529629 & 1.66370653 & ๑. 18638444 \\
\hline C & -2.82424854 & 3.77610983 & 1.15599757 \\
\hline C & -4.07892617 & 3.14532513 & 1. 12070358 \\
\hline $\mathrm{N}$ & -5.11153996 & 3.55353101 & 2.01507491 \\
\hline 0 & -4.87552715 & 4.45420710 & 2.81536748 \\
\hline 0 & -6.20262824 & 2.99084749 & 1.95706632 \\
\hline $\mathrm{H}$ & -2.65792939 & 4.57144584 & 1.87669389 \\
\hline C & -1.44841326 & -1.05243563 & -0.62976715 \\
\hline $\mathrm{H}$ & -1.25563359 & -0.06640838 & -1.05455478 \\
\hline C & -0.38325844 & -1.77005067 & -0.16103523 \\
\hline $\mathrm{H}$ & -0.54340637 & -2.76921519 & ๑. 24525901 \\
\hline C & $\odot .97234524$ & -1.32893251 & -0.14475087 \\
\hline C & 1.37003465 & -0.03222730 & -0.53837676 \\
\hline C & 1.97623656 & -2.21102171 & $\odot .29764563$ \\
\hline C & 2.68811001 & 0.34323118 & -0.49567135 \\
\hline $\mathrm{H}$ & 0.62906315 & 0.70309219 & -0.85553816 \\
\hline $\mathrm{C}$ & 3.29800909 & -1.84259600 & $\odot .34233579$ \\
\hline $\mathrm{H}$ & 1.69816404 & -3.21990252 & ๑. 59920437 \\
\hline
\end{tabular}




$\begin{array}{lrrr}\mathrm{C} & 3.68773191 & -0.54954490 & -0.05482407 \\ \mathrm{H} & 2.96491756 & 1.35406457 & -0.78150189 \\ \mathrm{H} & 4.04899977 & -2.55437502 & 0.67255949 \\ \mathrm{~N} & 5.01081781 & -0.15893114 & -0.00888333 \\ \mathrm{C} & 5.96241072 & -0.83702415 & 0.80612446 \\ \mathrm{C} & 7.15364361 & -1.28547890 & 0.24332081 \\ \mathrm{C} & 5.72563667 & -1.01941721 & 2.16606058 \\ \mathrm{C} & 8.09828313 & -1.91884565 & 1.03614953 \\ \mathrm{H} & 7.33260032 & -1.13209551 & -0.81902946 \\ \mathrm{C} & 6.66913798 & -1.66486738 & 2.95049278 \\ \mathrm{H} & 4.80049779 & -0.64926567 & 2.60317839 \\ \mathrm{C} & 7.85765746 & -2.11494706 & 2.38955062 \\ \mathrm{H} & 9.02676456 & -2.26774139 & 0.59074584 \\ \mathrm{H} & 6.47989418 & -1.80367644 & 4.01222514 \\ \mathrm{H} & 8.59835868 & -2.61438603 & 3.00893287 \\ \mathrm{C} & 5.48961626 & 0.93195894 & -0.79292501 \\ \mathrm{C} & 5.30489634 & 0.94182127 & -2.17237821 \\ \mathrm{C} & 6.17942314 & 1.97047521 & -0.17587604 \\ \mathrm{C} & 5.79653437 & 1.99608889 & -2.92665041 \\ \mathrm{H} & 4.77438651 & 0.11891848 & -2.64740282 \\ \mathrm{C} & 6.67823745 & 3.01629836 & -0.93739672 \\ \mathrm{H} & 6.32019793 & 1.95100006 & 0.90291971 \\ \mathrm{C} & 6.48594786 & 3.03391530 & -2.31256177 \\ \mathrm{H} & 5.64942883 & 2.00061931 & -4.00384108 \\ \mathrm{H} & 7.21391290 & 3.82758969 & -0.45062625 \\ \mathrm{H} & 6.87451031 & 3.85687879 & -2.90707787\end{array}$

6b, IEF-PCM (chloroform) /M06/6-311G(d), E=-1222.975016162796

$\begin{array}{lrrr}\mathrm{C} & 0.41223551 & 2.02250392 & 0.24659855 \\ \mathrm{C} & 1.28169196 & 2.28196259 & -0.79968651 \\ \mathrm{C} & 1.24309524 & 3.51061763 & -1.42935699 \\ \mathrm{C} & 0.32141321 & 4.45001259 & -0.97541541 \\ \mathrm{C} & -0.54156599 & 4.16308548 & 0.07875282 \\ \mathrm{C} & -0.51653886 & 2.92924766 & 0.71996342 \\ \mathrm{C} & 1.63325876 & 0.14164800 & 0.03990534 \\ \mathrm{C} & 2.13425946 & 1.06943367 & -1.04150753 \\ \mathrm{H} & 1.91224836 & 3.74307672 & -2.25566882 \\ \mathrm{H} & 0.27247857 & 5.42516145 & -1.45303898 \\ \mathrm{H} & -1.25382826 & 4.91506159 & 0.40699366 \\ \mathrm{H} & -1.19642810 & 2.68676348 & 1.53740520 \\ \mathrm{~N} & 0.67269224 & 0.70059485 & 0.71954259 \\ \mathrm{C} & 3.62418919 & 1.35168966 & -0.84111241 \\ \mathrm{H} & 4.21460410 & 0.43643192 & -0.95489016 \\ \mathrm{H} & 3.96064587 & 2.07218630 & -1.59369591 \\ \mathrm{H} & 3.82422681 & 1.77695280 & 0.14885025 \\ \mathrm{C} & 1.87087788 & 0.48146112 & -2.43303825 \\ \mathrm{H} & 2.08901810 & 1.24647885 & -3.18517941 \\ \mathrm{H} & 2.51785050 & -0.37655857 & -2.63652580 \\ \mathrm{H} & 0.82361283 & 0.17995744 & -2.55382534 \\ \mathrm{O} & -2.52200620 & 1.35281710 & 2.66480925 \\ \mathrm{C} & -0.19305019 & 0.05595503 & 1.73038796 \\ \mathrm{H} & -0.27594626 & 0.73305229 & 2.58591944 \\ \mathrm{H} & 0.31299433 & -0.85843900 & 2.04798870 \\ \mathrm{C} & -1.55145930 & -0.19324063 & 1.15911093 \\ \mathrm{C} & -2.67197502 & 0.51720103 & 1.74839061 \\ \mathrm{C} & -3.95797997 & 0.19805717 & 1.17848555 \\ \mathrm{H} & -4.82064890 & 0.70282495 & 1.60931526 \\ \mathrm{C} & -1.71540188 & -1.05777522 & 0.10754244 \\ \mathrm{H} & -0.86391750 & -1.57656174 & -0.33079812 \\ \mathrm{C} & -4.10614243 & -0.66583450 & 0.13368789 \\ \mathrm{C} & -2.98084210 & -1.29853542 & -0.42632064 \\ \mathrm{~N} & -3.12161742 & -2.17734511 & -1.53778400 \\ \mathrm{O} & -4.24616178 & -2.39650269 & -1.97794579 \\ \mathrm{O} & -2.11099271 & -2.68332146 & -2.02461817 \\ \mathrm{H} & -5.08525920 & -0.87085878 & -0.28976557 \\ \mathrm{C} & 2.20599659 & -1.16695860 & 0.35551943\end{array}$




$\begin{array}{lrrr}\mathrm{C} & 2.15023991 & -2.22866749 & -0.54839857 \\ \mathrm{C} & 2.86608256 & -1.32622291 & 1.57700098 \\ \mathrm{C} & 2.73813973 & -3.44065267 & -0.22120866 \\ \mathrm{H} & 1.61798340 & -2.12468229 & -1.49034577 \\ \mathrm{C} & 3.46695231 & -2.53509976 & 1.88624284 \\ \mathrm{H} & 2.92101344 & -0.49147636 & 2.27336532 \\ \mathrm{C} & 3.40015624 & -3.59293602 & 0.98941281 \\ \mathrm{H} & 2.67354323 & -4.27209845 & -0.91741100 \\ \mathrm{H} & 3.98540805 & -2.65177069 & 2.83401205 \\ \mathrm{H} & 3.86436565 & -4.54390743 & 1.23728291\end{array}$

7b, IEF-PCM (chloroform) /M06/6-311G(d), E=-992.0500559452157

$\begin{array}{lrrr}\mathrm{C} & 1.80051362 & 0.63453186 & 0.14537557 \\ \mathrm{C} & 2.71766901 & -0.24978296 & -0.40682155 \\ \mathrm{C} & 3.54286941 & 0.17777761 & -1.42704006 \\ \mathrm{C} & 3.41613247 & 1.49465895 & -1.86203186 \\ \mathrm{C} & 2.48465958 & 2.35868166 & -1.29252932 \\ \mathrm{C} & 1.64516228 & 1.94377856 & -0.26399681 \\ \mathrm{C} & 1.53122370 & -1.27003770 & 1.28202384 \\ \mathrm{C} & 2.58661368 & -1.58297481 & 0.27760663 \\ \mathrm{H} & 4.27090245 & -0.49140827 & -1.88113952 \\ \mathrm{H} & 4.05474855 & 1.85531999 & -2.66421900 \\ \mathrm{H} & 2.40662710 & 3.37814919 & -1.66003395 \\ \mathrm{H} & 0.89790581 & 2.60201543 & 0.18074513 \\ \mathrm{~N} & 1.09924750 & -0.05933310 & 1.18180538 \\ \mathrm{C} & 3.87880279 & -2.04840118 & 0.94782352 \\ \mathrm{H} & 3.72192062 & -2.98491761 & 1.49282134 \\ \mathrm{H} & 4.63931254 & -2.22661004 & 0.18038966 \\ \mathrm{H} & 4.26625666 & -1.29714711 & 1.64293140 \\ \mathrm{C} & 2.04306012 & -2.65829427 & -\odot .67498345 \\ \mathrm{H} & 2.77379038 & -2.82860605 & -1.47241020 \\ \mathrm{H} & 1.88295020 & -3.60399183 & -\odot .14714174 \\ \mathrm{H} & 1.09925530 & -2.34828008 & -1.13665432 \\ \mathrm{O} & -1.02867700 & 3.00590316 & 1.04454494 \\ \mathrm{C} & -0.04119805 & 0.51621093 & 1.92662865 \\ \mathrm{H} & 0.26173854 & 1.50492137 & 2.28462219 \\ \mathrm{H} & -0.20369306 & -0.14293330 & 2.78491031 \\ \mathrm{C} & -1.23743032 & 0.64840993 & 1.04432567 \\ \mathrm{C} & -1.65775307 & 1.98997230 & 0.68073270 \\ \mathrm{C} & -2.84304033 & 2.05208675 & -0.13868998 \\ \mathrm{H} & -3.19086716 & 3.04436428 & -0.41958195 \\ \mathrm{C} & -1.90967499 & -0.46574045 & 0.60738574 \\ \mathrm{H} & -1.58003633 & -1.46651746 & 0.88607682 \\ \mathrm{C} & -3.50072576 & 0.93677380 & -0.56544703 \\ \mathrm{C} & -3.03744338 & -0.34175623 & -0.20237081 \\ \mathrm{~N} & -3.71817557 & -1.51059133 & -0.65203476 \\ \mathrm{O} & -4.71470680 & -1.37749349 & -1.35528045 \\ \mathrm{O} & -3.28298342 & -2.61214205 & -0.32218699 \\ \mathrm{H} & -4.38481420 & 1.01153520 & -1.19210878 \\ \mathrm{H} & 1.11079235 & -1.96193222 & 2.00782947\end{array}$

8b, IEF-PCM (chlor oform) /M06/6-311G(d), E=-1434.251395889676

$\begin{array}{lllr}\mathrm{C} & 3.13059692 & -1.55366421 & -0.65793000 \\ \mathrm{C} & 2.89078640 & -2.63098682 & 0.18373717 \\ \mathrm{C} & 3.93760270 & -3.42085315 & 0.61210239 \\ \mathrm{C} & 5.22666234 & -3.10889544 & 0.18621691 \\ \mathrm{C} & 5.45140365 & -2.02271448 & -0.65153042 \\ \mathrm{C} & 4.40282938 & -1.22129914 & -1.09019709 \\ \mathrm{C} & 0.88449992 & -1.53224294 & -0.32542381 \\ \mathrm{C} & 1.41649316 & -2.70824796 & 0.48157263 \\ \mathrm{H} & 3.76475786 & -4.26861239 & 1.27306652 \\ \mathrm{H} & 6.06552001 & -3.71691158 & 0.51430562 \\ \mathrm{H} & 6.46400966 & -1.78898531 & -0.96929897 \\ \mathrm{H} & 4.59503347 & -0.37331418 & -1.74152649 \\ \mathrm{~N} & 1.90068167 & -0.92748017 & -0.94986515 \\ \mathrm{C} & 1.17996742 & -2.51045261 & 1.98174379 \\ \mathrm{H} & 0.11898417 & -2.55283161 & 2.24374223\end{array}$




$\begin{array}{rrrr}\mathrm{H} & 1.69374743 & -3.30099433 & 2.53954280 \\ \mathrm{H} & 1.57588242 & -1.54675924 & 2.31930227 \\ \mathrm{C} & 0.84686590 & -4.03970885 & -0.01393713 \\ \mathrm{H} & 1.34686443 & -4.86631233 & 0.50278429 \\ \mathrm{H} & -0.22633160 & -4.13023656 & 0.17560426 \\ \mathrm{H} & 1.01419522 & -4.16477440 & -1.08904498 \\ \mathrm{O} & -0.62751286 & 1.73788243 & -1.87623369 \\ \mathrm{C} & 1.79085306 & 0.26937752 & -1.80211513 \\ \mathrm{H} & 0.95494560 & 0.12445694 & -2.49231776 \\ \mathrm{H} & 2.70301682 & 0.29716657 & -2.40382621 \\ \mathrm{C} & 1.58267255 & 1.54222240 & -1.03963408 \\ \mathrm{C} & 0.30235260 & 2.21145472 & -1.17908583 \\ \mathrm{C} & 0.17175016 & 3.44841799 & -0.45570001 \\ \mathrm{H} & -0.77951312 & 3.96952301 & -0.54821634 \\ \mathrm{C} & 2.58355898 & 2.07228606 & -0.26451383 \\ \mathrm{H} & 3.54508527 & 1.57037116 & -0.17080763 \\ \mathrm{C} & 1.17550614 & 3.96011158 & 0.31391333 \\ \mathrm{C} & 2.39653613 & 3.27407003 & 0.42259928 \\ \mathrm{~N} & 3.45010854 & 3.80843008 & 1.21984989 \\ \mathrm{O} & 3.26612709 & 4.87504929 & 1.80026473 \\ \mathrm{O} & 4.50734623 & 3.18679485 & 1.30095801 \\ \mathrm{H} & 1.05014022 & 4.89544875 & 0.85164420 \\ \mathrm{C} & -0.42951331 & -1.06144612 & -0.44024093 \\ \mathrm{H} & -0.57871861 & -0.17430836 & -1.05582290 \\ \mathrm{C} & -1.52427149 & -1.60805973 & 0.17698577 \\ \mathrm{H} & -1.40624613 & -2.51258675 & 0.77370167 \\ \mathrm{C} & -2.84930962 & -1.09832187 & 0.12980764 \\ \mathrm{C} & -3.20066413 & 0.10253114 & -0.52971660 \\ \mathrm{C} & -3.87960316 & -1.79642221 & 0.79088788 \\ \mathrm{C} & -4.49060046 & 0.55983830 & -0.52733550 \\ \mathrm{H} & -2.43909508 & 0.69561165 & -1.03816845 \\ \mathrm{C} & -5.17513314 & -1.35046014 & 0.79921408 \\ \mathrm{H} & -3.63679082 & -2.72039635 & 1.31444302 \\ \mathrm{C} & -5.52649212 & -0.14988136 & 0.13434333 \\ \mathrm{H} & -4.71023147 & 1.49099497 & -1.03881011 \\ \mathrm{H} & -5.92800771 & -1.92761389 & 1.32505120 \\ \mathrm{H} & -6.80209027 & 0.30687019 & 0.13428056 \\ \mathrm{H} & -7.83777480 & -0.41783888 & 0.84198909 \\ \mathrm{H} & -7.96375581 & -1.43329968 & 0.44638184 \\ \mathrm{H} & -7.62012732 & -0.49141892 & 1.91493071 \\ \mathrm{H} & -8.78673728 & 0.10535754 & 0.72804419 \\ \mathrm{C} & -7.14081440 & 1.54053575 & -0.54737848 \\ \mathrm{H} & -6.61234507 & 2.40104794 & -0.11841992 \\ \mathrm{H} & -6.90247551 & 1.48911295 & -1.61646220 \\ \mathrm{H} & -8.21095529 & 1.72161352 & -0.45183865\end{array}$

9b, IEF-PCM (chloroform) /M06/6-311G(d), E=-1504.806210729824

$\begin{array}{lrrr}\mathrm{C} & -2.91667922 & -1.66491426 & -0.63402686 \\ \mathrm{C} & -2.63282216 & -2.74408773 & 0.18955581 \\ \mathrm{C} & -3.65043567 & -3.57492696 & 0.61267533 \\ \mathrm{C} & -4.94889673 & -3.29517371 & 0.19680049 \\ \mathrm{C} & -5.21654495 & -2.20498282 & -0.62544291 \\ \mathrm{C} & -4.19895453 & -1.36449353 & -1.05921716 \\ \mathrm{C} & -0.68185303 & -1.56097591 & -0.30615015 \\ \mathrm{C} & -1.15608781 & -2.77456485 & 0.47179012 \\ \mathrm{H} & -3.44785321 & -4.42632285 & 1.25971820 \\ \mathrm{H} & -5.76648490 & -3.93323238 & 0.52102302 \\ \mathrm{H} & -6.23840807 & -2.00128018 & -0.93255173 \\ \mathrm{H} & -4.42117288 & -0.51405287 & -1.69726595 \\ \mathrm{~N} & -1.70520043 & -0.98887844 & -0.91713158 \\ \mathrm{C} & -0.54105871 & -4.06514223 & -0.08115983 \\ \mathrm{H} & 0.53600918 & -4.12231560 & 0.09662525 \\ \mathrm{H} & -1.00659328 & -4.92566245 & 0.41062151 \\ \mathrm{H} & -0.71578534 & -4.15772069 & -1.15817622 \\ \mathrm{C} & -0.90350830 & -2.61757035 & 1.97474474 \\ \mathrm{H} & -1.38114176 & -3.44519435 & 2.50941092\end{array}$




$\begin{array}{lrrr}\mathrm{H} & 0.16153604 & -2.63362920 & 2.22119551 \\ \mathrm{H} & -1.32829109 & -1.68183829 & 2.35233930 \\ \mathrm{O} & 0.67685582 & 1.81125621 & -1.57558379 \\ \mathrm{C} & -1.65790622 & 0.22654884 & -1.76579071 \\ \mathrm{H} & -2.54045615 & 0.17112779 & -2.40713725 \\ \mathrm{H} & -0.78025535 & 0.15131893 & -2.41239932 \\ \mathrm{C} & -1.59905934 & 1.50110546 & -0.98436088 \\ \mathrm{C} & -0.34659075 & 2.23280439 & -0.98107059 \\ \mathrm{C} & -0.35879008 & 3.47023097 & -0.24959713 \\ \mathrm{H} & 0.56924564 & 4.03831122 & -0.23411981 \\ \mathrm{C} & -2.71066611 & 1.98423554 & -0.33596474 \\ \mathrm{H} & -3.65296474 & 1.44008896 & -0.35611173 \\ \mathrm{C} & -1.46794884 & 3.93046840 & 0.39827420 \\ \mathrm{C} & -2.65959650 & 3.18857903 & 0.36459081 \\ \mathrm{~N} & -3.82666212 & 3.67073053 & 1.03290735 \\ \mathrm{O} & -3.75986947 & 4.73621584 & 1.63596002 \\ \mathrm{O} & -4.85495066 & 3.00173652 & 0.98090341 \\ \mathrm{H} & -1.44952604 & 4.86875868 & 0.94489796 \\ \mathrm{C} & 0.62896328 & -1.01758989 & -0.40314672 \\ \mathrm{H} & 0.72571886 & -0.09080085 & -0.97287109 \\ \mathrm{C} & 1.72925361 & -1.54308716 & 0.17955170 \\ \mathrm{H} & 1.66362273 & -2.47642714 & 0.73712156 \\ \mathrm{C} & 3.05567011 & -0.95786835 & 0.13523262 \\ \mathrm{C} & 4.11516872 & -1.68252944 & 0.69849547 \\ \mathrm{C} & 3.30949800 & 0.30379814 & -0.42845783 \\ \mathrm{C} & 5.40151978 & -1.18070640 & 0.69659892 \\ \mathrm{H} & 3.92180214 & -2.65582030 & 1.14395958 \\ \mathrm{C} & 4.59257894 & 0.81271697 & -0.43088554 \\ \mathrm{H} & 2.50219874 & 0.90298260 & -0.85096926 \\ \mathrm{C} & 5.61772816 & 0.06359431 & 0.12833607 \\ \mathrm{H} & 6.22898147 & -1.73262525 & 1.12800421 \\ \mathrm{H} & 4.80906501 & 1.78651839 & -0.85647682 \\ \mathrm{~N} & 6.98547047 & 0.61400425 & 0.12455998 \\ \mathrm{O} & 7.15441795 & 1.69770484 & -0.39329018 \\ \mathrm{O} & 7.86074733 & -0.05016207 & 0.63983059\end{array}$

$10 \mathrm{~b}, \quad \mathrm{IEF}-\mathrm{PCM}$ (chloroform) /M06/6-311G(d), E=-1413.638407385316

$\begin{array}{lrrr}\mathrm{C} & -2.63359276 & -1.66947759 & -0.64780976 \\ \mathrm{C} & -2.35577564 & -2.74659763 & 0.18041505 \\ \mathrm{C} & -3.37505017 & -3.58140432 & 0.59140904 \\ \mathrm{C} & -4.66952636 & -3.30863339 & 0.15847203 \\ \mathrm{C} & -4.93116775 & -2.22110441 & -0.66889088 \\ \mathrm{C} & -3.91162976 & -1.37633688 & -1.09024651 \\ \mathrm{C} & -0.40267287 & -1.55451053 & -0.29074060 \\ \mathrm{C} & -0.88205446 & -2.77173368 & 0.47995805 \\ \mathrm{H} & -3.17685201 & -4.43098946 & 1.24230375 \\ \mathrm{H} & -5.48826093 & -3.95006106 & 0.47309520 \\ \mathrm{H} & -5.94975207 & -2.02263227 & -0.99016122 \\ \mathrm{H} & -4.12933905 & -0.52795664 & -1.73264248 \\ \mathrm{~N} & -1.42228454 & -0.98763006 & -0.91547676 \\ \mathrm{C} & -0.25752651 & -4.05925677 & -0.06951837 \\ \mathrm{H} & 0.81789415 & -4.11366375 & 0.11886326 \\ \mathrm{H} & -0.72560074 & -4.92257054 & 0.41505757 \\ \mathrm{H} & -0.42096414 & -4.14945269 & -1.14853945 \\ \mathrm{C} & -0.64718500 & -2.61824082 & 1.98579075 \\ \mathrm{H} & -1.12953739 & -3.44786317 & 2.51325431 \\ \mathrm{H} & 0.41496553 & -2.63271904 & 2.24461840 \\ \mathrm{H} & -1.07802288 & -1.68406207 & 2.36040092 \\ \mathrm{O} & 0.95179581 & 1.82813382 & -1.55839230 \\ \mathrm{C} & -1.36927209 & 0.22649976 & -1.76396718 \\ \mathrm{H} & -2.24274577 & 0.16673803 & -2.41738178 \\ \mathrm{H} & -0.48244718 & 0.15512493 & -2.39856500 \\ \mathrm{C} & -1.32636374 & 1.50317561 & -0.98456145 \\ \mathrm{C} & -0.07861094 & 2.24366929 & -0.97333223 \\ \mathrm{C} & -0.10547826 & 3.48305877 & -0.24463764 \\ \mathrm{H} & 0.81874701 & 4.05712607 & -0.22290449\end{array}$




$\begin{array}{lrrr}\mathrm{C} & -2.44604498 & 1.98007667 & -0.34617555 \\ \mathrm{H} & -3.38426571 & 1.42915953 & -0.37232532 \\ \mathrm{C} & -1.22288059 & 3.93723680 & 0.39287542 \\ \mathrm{C} & -2.40932007 & 3.18696111 & 0.35196271 \\ \mathrm{~N} & -3.58421490 & 3.66230112 & 1.00961728 \\ \mathrm{O} & -3.53064262 & 4.73120148 & 1.60870953 \\ \mathrm{O} & -4.60725087 & 2.98502474 & 0.95365363 \\ \mathrm{H} & -1.21528979 & 4.87702377 & 0.93725845 \\ \mathrm{C} & 0.90507764 & -1.00622373 & -0.37014098 \\ \mathrm{H} & 1.00596602 & -0.07741744 & -0.93536637 \\ \mathrm{C} & 2.00191705 & -1.53229770 & 0.22248380 \\ \mathrm{H} & 1.92910415 & -2.46812602 & 0.77527019 \\ \mathrm{C} & 3.32747350 & -0.94996838 & 0.19650600 \\ \mathrm{C} & 4.37676257 & -1.67524645 & 0.77715287 \\ \mathrm{C} & 3.59237952 & 0.31228434 & -0.36895343 \\ \mathrm{C} & 5.66143913 & -1.16676413 & 0.78740446 \\ \mathrm{H} & 4.17233514 & -2.64727880 & 1.22171370 \\ \mathrm{C} & 4.87501964 & 0.81431903 & -0.35050367 \\ \mathrm{H} & 2.78939477 & 0.90701111 & -0.80566022 \\ \mathrm{C} & 5.91576975 & 0.08002821 & 0.22377845 \\ \mathrm{H} & 6.47556207 & -1.73267862 & 1.23735306 \\ \mathrm{H} & 5.09706221 & 1.78959954 & -0.77693503 \\ \mathrm{C} & 7.28721725 & 0.62078141 & 0.24464354 \\ \mathrm{O} & 7.61072315 & 1.68667162 & -0.21250823 \\ \mathrm{H} & 8.03711398 & -0.04702976 & 0.73036425\end{array}$

11 b, IEF-PCM (chloroform) /M06/6-311G(d), E=-1777.529621498088

$\begin{array}{lrrr}\mathrm{C} & -4.51464622 & -1.50825641 & -0.73545571 \\ \mathrm{C} & -4.17942535 & -2.60669197 & 0.03890698 \\ \mathrm{C} & -5.16766996 & -3.46338363 & 0.48079781 \\ \mathrm{C} & -6.48746946 & -3.18493374 & 0.13582295 \\ \mathrm{C} & -6.80493031 & -2.06731519 & -0.63016475 \\ \mathrm{C} & -5.81736669 & -1.20016552 & -1.08189457 \\ \mathrm{C} & -2.26330011 & -1.37182746 & -0.50293380 \\ \mathrm{C} & -2.69024594 & -2.61657897 & 0.26292790 \\ \mathrm{H} & -4.92688406 & -4.33230329 & 1.09061757 \\ \mathrm{H} & -7.28219113 & -3.84336149 & 0.47633281 \\ \mathrm{H} & -7.84335492 & -1.86181127 & -0.87546766 \\ \mathrm{H} & -6.06777060 & -0.31644788 & -1.66205335 \\ \mathrm{~N} & -3.33159369 & -0.80853968 & -1.05990058 \\ \mathrm{C} & -2.07869918 & -3.88973947 & -0.32852987 \\ \mathrm{H} & -0.99472461 & -3.93628545 & -0.19345092 \\ \mathrm{H} & -2.51401901 & -4.76457912 & 0.16641093 \\ \mathrm{H} & -2.29244010 & -3.96863143 & -1.39967345 \\ \mathrm{C} & -2.39304806 & -2.47705403 & 1.76057631 \\ \mathrm{H} & -2.84699488 & -3.31511969 & 2.29987009 \\ \mathrm{H} & -1.32102948 & -2.48550602 & 1.97529122 \\ \mathrm{H} & -2.81193189 & -1.54995639 & 2.16758415 \\ \mathrm{O} & -3.75276752 & 2.90009986 & -2.90932485 \\ \mathrm{C} & -3.36638467 & 0.43988683 & -1.81087029 \\ \mathrm{H} & -4.14567736 & 0.34802586 & -2.57677199 \\ \mathrm{H} & -2.43460604 & 0.54762578 & -2.37604306 \\ \mathrm{C} & -3.62280657 & 1.62818636 & -0.93656363 \\ \mathrm{C} & -3.80572950 & 2.87204287 & -1.66527934 \\ \mathrm{C} & -4.04488241 & 4.03250641 & -0.84381867 \\ \mathrm{H} & -4.18415835 & 4.97963304 & -1.36232846 \\ \mathrm{C} & -3.68110655 & 1.57321638 & 0.42595883 \\ \mathrm{H} & -3.55615360 & 0.63849875 & 0.96868562 \\ \mathrm{C} & -4.09792138 & 3.96586044 & 0.51818280 \\ \mathrm{C} & -3.91763546 & 2.73563192 & 1.17466054 \\ \mathrm{~N} & -3.97498643 & 2.65820995 & 2.58857198 \\ \mathrm{O} & -4.18511735 & 3.68534855 & 3.23297472 \\ \mathrm{O} & -3.81229250 & 1.56421638 & 3.13481577 \\ \mathrm{H} & -4.28039731 & 4.85382609 & 1.11683256 \\ \mathrm{C} & -0.97539261 & -0.81266668 & -0.63633075 \\ \mathrm{H} & -0.88065065 & 0.11786695 & -1.18576753\end{array}$




$\begin{array}{lrrr}\mathrm{C} & 0.15730794 & -1.35745847 & -0.10508696 \\ \mathrm{H} & 0.07671297 & -2.28133110 & 0.46647882 \\ \mathrm{C} & 1.49095341 & -0.86734666 & -0.19606692 \\ \mathrm{C} & 2.51057301 & -1.55141039 & 0.43934183 \\ \mathrm{C} & 1.81402529 & 0.33961387 & -0.95429962 \\ \mathrm{C} & 3.83916349 & -1.13020955 & 0.38696485 \\ \mathrm{H} & 2.26830411 & -2.45094304 & 1.00651660 \\ \mathrm{C} & 4.92036906 & -1.78208930 & 1.01882560 \\ \mathrm{C} & 4.13440537 & 0.03482699 & -0.34964386 \\ \mathrm{C} & 6.19333256 & -1.30668148 & 0.92168664 \\ \mathrm{H} & 4.72293014 & -2.68814794 & 1.58832737 \\ \mathrm{C} & 5.40724087 & 0.53454579 & -0.45958782 \\ \mathrm{C} & 6.48396043 & -0.12321595 & 0.17401593 \\ \mathrm{H} & 6.99321725 & -1.85236027 & 1.40911144 \\ \mathrm{H} & 5.53961682 & 1.44929443 & -1.02591759 \\ \mathrm{O} & 1.05244859 & 1.03718459 & -1.56332939 \\ \mathrm{O} & 3.14466076 & 0.71149872 & -0.97637161 \\ \mathrm{~N} & 7.74813793 & 0.34560386 & 0.07588097 \\ \mathrm{C} & 8.08484183 & 1.48833341 & -0.76775770 \\ \mathrm{H} & 9.11939942 & 1.34797647 & -1.10209957 \\ \mathrm{H} & 7.47790406 & 1.46064643 & -1.67858334 \\ \mathrm{C} & 8.86181352 & -0.25061990 & 0.80800068 \\ \mathrm{H} & 9.59046019 & 0.54812779 & 0.98901961 \\ \mathrm{H} & 8.52308463 & -0.56493778 & 1.80045329 \\ \mathrm{C} & 7.93919289 & 2.81188089 & -0.04327398 \\ \mathrm{H} & 8.22644075 & 3.64344856 & -0.69418772 \\ \mathrm{H} & 6.90815829 & 2.98043183 & 0.28463489 \\ \mathrm{H} & 8.57960264 & 2.84479888 & 0.84512567 \\ \mathrm{C} & 9.51176724 & -1.39440445 & 0.05479698 \\ \mathrm{H} & 10.35979592 & -1.79660329 & 0.61780675 \\ \mathrm{H} & 8.80444851 & -2.21161713 & -0.12236295 \\ \mathrm{H} & 9.88483411 & -1.06084558 & -0.91982664\end{array}$

12 b, IEF-PCM (chloroform) /M06/6-311G(d), E=-1624.701107513979

$\begin{array}{lrrr}\mathrm{C} & -4.06469740 & -0.88390065 & -0.97710561 \\ \mathrm{C} & -4.11838738 & -2.06509019 & -0.25436490 \\ \mathrm{C} & -5.33971587 & -2.64282696 & 0.02761999 \\ \mathrm{C} & -6.49387742 & -2.00541521 & -0.42048083 \\ \mathrm{C} & -6.41958547 & -0.81025668 & -1.12870356 \\ \mathrm{C} & -5.19447195 & -0.22190091 & -1.42085944 \\ \mathrm{C} & -1.89744025 & -1.37762045 & -0.52971979 \\ \mathrm{C} & -2.71923493 & -2.48895602 & 0.10733696 \\ \mathrm{H} & -5.40417688 & -3.57118659 & 0.59236577 \\ \mathrm{H} & -7.46608304 & -2.44192404 & -0.20765892 \\ \mathrm{H} & -7.33348013 & -0.32287887 & -1.45728343 \\ \mathrm{H} & -5.14079997 & 0.71903572 & -1.96104322 \\ \mathrm{~N} & -2.71018193 & -0.52009980 & -1.14199155 \\ \mathrm{C} & -2.40925605 & -3.86067748 & -0.49798480 \\ \mathrm{H} & -1.40239445 & -4.20966934 & -0.25261090 \\ \mathrm{H} & -3.11917477 & -4.59644519 & -0.10492296 \\ \mathrm{H} & -2.51068501 & -3.84469448 & -1.58806737 \\ \mathrm{C} & -2.55377400 & -2.48681602 & 1.63146735 \\ \mathrm{H} & -3.26752371 & -3.18895374 & 2.07520418 \\ \mathrm{H} & -1.54942939 & -2.79282790 & 1.93702424 \\ \mathrm{H} & -2.74977292 & -1.49528841 & 2.05450719 \\ \mathrm{O} & -1.35997347 & 3.10804578 & -2.69742193 \\ \mathrm{C} & -2.30404109 & 0.71072872 & -1.80969929 \\ \mathrm{H} & -3.05930280 & 0.94013563 & -2.57034699 \\ \mathrm{H} & -1.38756145 & 0.52485879 & -2.38115706 \\ \mathrm{C} & -2.12049117 & 1.85414127 & -0.85976966 \\ \mathrm{C} & -1.60513737 & 3.06442879 & -1.47626142 \\ \mathrm{C} & -1.40200745 & 4.17038336 & -0.57422477 \\ \mathrm{H} & -1.01410360 & 5.09104099 & -1.00689960 \\ \mathrm{C} & -2.39372606 & 1.78206230 & 0.47622881 \\ \mathrm{H} & -2.78487190 & 0.87557366 & 0.93351163 \\ \mathrm{C} & -1.67577234 & 4.08560939 & 0.76049570\end{array}$




$\begin{array}{lrrr}\mathrm{C} & -2.17557614 & 2.89067586 & 1.30628748 \\ \mathrm{~N} & -2.44578204 & 2.78965290 & 2.69543794 \\ \mathrm{O} & -2.24900511 & 3.76960752 & 3.41217366 \\ \mathrm{O} & -2.86844887 & 1.72237532 & 3.14498376 \\ \mathrm{H} & -1.51137640 & 4.93143306 & 1.42189544 \\ \mathrm{C} & -0.50456880 & -1.17613054 & -0.48489081 \\ \mathrm{H} & -0.13460895 & -0.25684090 & -0.93147363 \\ \mathrm{C} & 0.39023985 & -2.02455020 & 0.09807896 \\ \mathrm{H} & 0.02050999 & -2.94594284 & 0.54587578 \\ \mathrm{C} & 1.80441729 & -1.84315327 & 0.19478892 \\ \mathrm{C} & 2.56845199 & -2.86825387 & 0.80415568 \\ \mathrm{C} & 2.46187703 & -0.69367972 & -0.27838889 \\ \mathrm{C} & 3.93323845 & -2.78631290 & 0.94704962 \\ \mathrm{H} & 2.04982019 & -3.75175452 & 1.17150654 \\ \mathrm{C} & 3.82714549 & -0.58683377 & -0.14255594 \\ \mathrm{H} & 1.90402425 & 0.11720395 & -0.74192171 \\ \mathrm{C} & 4.56796720 & -1.63744736 & 0.46969481 \\ \mathrm{H} & 4.48560623 & -3.59132858 & 1.42313944 \\ \mathrm{C} & 4.78212111 & 0.43317483 & -0.50206380 \\ \mathrm{C} & 6.04153861 & -0.04469098 & -0.09589596 \\ \mathrm{C} & 4.68139916 & 1.67773034 & -1.11432356 \\ \mathrm{C} & 7.20361215 & 0.69044473 & -0.28519189 \\ \mathrm{C} & 5.83605971 & 2.41824605 & -1.30865448 \\ \mathrm{H} & 3.71552254 & 2.06469919 & -1.43275544 \\ \mathrm{C} & 7.07891223 & 1.92846624 & -0.89790764 \\ \mathrm{H} & 8.17427777 & 0.31901687 & 0.03404699 \\ \mathrm{H} & 5.77683470 & 3.39399466 & -1.78377330 \\ \mathrm{H} & 7.96905488 & 2.53115600 & -1.06075358 \\ \mathrm{~N} & 5.88958067 & -1.29874949 & 0.48588981 \\ \mathrm{C} & 6.98345325 & -2.07542418 & 1.01544360 \\ \mathrm{H} & 7.44979005 & -1.56389438 & 1.86424626 \\ \mathrm{H} & 6.62030537 & -3.04619480 & 1.35328847 \\ \mathrm{H} & 7.74166895 & -2.24109712 & 0.24323990\end{array}$

Acidly open $(b+)$

$\begin{array}{lrrr}\text { 1 b }+, \text { IEF-PCM(chloroform)/M06/6-311G }(\mathrm{d}), \quad E=-1038.58545028827 \\ \mathrm{C} & -3.65524503 & -1.13229169 & -0.00513550 \\ \mathrm{C} & -3.75773747 & 0.22971342 & -0.23906774 \\ \mathrm{C} & -4.97255679 & 0.88092622 & -0.35234376 \\ \mathrm{C} & -6.11746503 & 0.10100396 & -0.22920029 \\ \mathrm{C} & -6.03487008 & -1.26823227 & -0.00179495 \\ \mathrm{C} & -4.79754429 & -1.89763810 & 0.11324663 \\ \mathrm{C} & -2.20011128 & -1.51525239 & 0.07762069 \\ \mathrm{C} & -1.51909197 & -0.16981262 & -0.14061871 \\ \mathrm{H} & -5.04675132 & 1.95116970 & -0.52537235 \\ \mathrm{H} & -7.09221923 & 0.57399926 & -0.31222548 \\ \mathrm{H} & -6.94636260 & -1.85261275 & 0.08896800 \\ \mathrm{H} & -4.73861807 & -2.96938964 & 0.29365975 \\ \mathrm{~N} & -2.45652736 & 0.76966474 & -0.33274662 \\ \mathrm{C} & -0.15361724 & 0.12867606 & -0.14800251 \\ \mathrm{H} & 0.11722961 & 1.17200053 & -0.29076771 \\ \mathrm{C} & 0.86289086 & -0.78265062 & 0.01423275 \\ \mathrm{H} & 0.60408309 & -1.82915335 & 0.16979545 \\ \mathrm{C} & 2.25161536 & -0.51523643 & -0.00009690 \\ \mathrm{C} & 3.16175573 & -1.57776990 & 0.19520634 \\ \mathrm{C} & 2.80450178 & 0.76932047 & -0.20847469 \\ \mathrm{C} & 4.51612695 & -1.38846695 & 0.18613181 \\ \mathrm{H} & 2.76978490 & -2.58057845 & 0.35716761 \\ \mathrm{C} & 4.15358129 & 0.97754055 & -0.22326710 \\ \mathrm{H} & 2.15290347 & 1.62529925 & -0.36601805 \\ \mathrm{C} & 5.06452121 & -0.09756933 & -0.02767951 \\ \mathrm{H} & 5.16770131 & -2.24149769 & 0.33960676 \\ \mathrm{H} & 4.52544113 & 1.98291677 & -0.38673634 \\ \mathrm{~N} & 6.39834054 & 0.10395564 & -0.04666588 \\ \mathrm{C} & 7.30949364 & -1.00467943 & 0.17168621 \\ \mathrm{H} & 7.20180681 & -1.77288320 & -0.60332212 \\ & & & \end{array}$




\begin{tabular}{|c|c|c|c|}
\hline $\mathrm{H}$ & 8.33465766 & $-\odot .63855906$ & $\odot .14371414$ \\
\hline $\mathrm{H}$ & 7. 14432946 & -1.47255148 & 1.14915941 \\
\hline C & 6.94325590 & 1.42782413 & $-\odot .28752385$ \\
\hline $\mathrm{H}$ & 6.65342804 & 2.13328949 & $\odot .50016695$ \\
\hline $\mathrm{H}$ & 8.03061695 & 1.37055394 & -0.30497450 \\
\hline $\mathrm{H}$ & 6.61199496 & 1.82775970 & -1.25263598 \\
\hline C & -2.22525093 & 2.18758605 & -0.55117956 \\
\hline $\mathrm{H}$ & -1.29516264 & 2.33234286 & -1.10636875 \\
\hline $\mathrm{H}$ & -3.02777810 & 2.57596428 & -1.18500284 \\
\hline C & -2.18375515 & 2.94854606 & 0.75782857 \\
\hline $\mathrm{H}$ & -1.38304379 & 2.53990798 & 1.39747733 \\
\hline $\mathrm{H}$ & -3.13689766 & 2.80847887 & 1.29333611 \\
\hline 0 & -1.95779996 & 4.28701653 & $\odot .41497846$ \\
\hline C & -1.85739184 & -2.51188977 & -1.03366903 \\
\hline $\mathrm{H}$ & -0.80766929 & -2.81673589 & $-1 . \odot 119 \odot \odot 17$ \\
\hline $\mathrm{H}$ & -2.46742544 & -3.41335040 & -0.91288046 \\
\hline $\mathrm{H}$ & -2.07111142 & -2.09123737 & -2.02175056 \\
\hline C & -1.89038525 & -2.08189411 & 1.46659979 \\
\hline $\mathrm{H}$ & -0.84322060 & -2.37815373 & 1.57181523 \\
\hline $\mathrm{H}$ & -2.11912889 & -1.35273096 & 2.25104290 \\
\hline $\mathrm{H}$ & -2.50678470 & -2.96960052 & 1.64303057 \\
\hline $\mathrm{H}$ & -1.97841835 & 4.82735709 & 1. 21008284 \\
\hline $2 b+$ & IEF-PCM (chloro & orm) / M०6/6-3 & $11 G(d), \quad E=-1503.960956857782$ \\
\hline & 4.49056454 & -0.72495624 & -0.23121849 \\
\hline C & 4.10506908 & $\odot .59085797$ & 0.02059634 \\
\hline C & 5.02530037 & 1.63182871 & -0.05418351 \\
\hline $\mathrm{C}$ & 6.32915282 & 1.31245629 & -0.37956772 \\
\hline C & 6.71619330 & $-\odot . \odot \odot 899768$ & -0.62341589 \\
\hline C & 5.80395978 & -1.04413585 & -0.55253967 \\
\hline $\mathrm{H}$ & 4.73402211 & 2.66312745 & 0.11996023 \\
\hline $\mathrm{H}$ & 7.06676661 & 2.10603766 & -0.45222120 \\
\hline $\mathrm{H}$ & $7.75000 \bullet 63$ & $-\odot .22616011$ & -0.87545886 \\
\hline $\mathrm{H}$ & 6.09997192 & -2.07112221 & -0.74369216 \\
\hline $\mathrm{C}$ & 2.09410248 & -0.46885006 & $\odot .29371940$ \\
\hline C & $\odot .67321245$ & -0.68480992 & $\odot .51687828$ \\
\hline C & -2.32763807 & 1.35112696 & -0.06191312 \\
\hline C & -0.19011237 & $\odot .17863431$ & -0.05241608 \\
\hline C & -3.73484933 & 1.39232909 & -0.10907248 \\
\hline C & -1.64329166 & $\odot .13081103$ & -0.04960838 \\
\hline $\mathrm{H}$ & $\odot .22015312$ & 1.02679259 & -0.59886719 \\
\hline C & -4.45644941 & 0.22043236 & -0.15028722 \\
\hline C & -2.38455877 & -1.05236078 & -0.10259671 \\
\hline C & -3.75847705 & -0.98687671 & -0.15012005 \\
\hline $\mathrm{H}$ & -5.53964471 & ๑. 20554133 & -0.18705928 \\
\hline $\mathrm{H}$ & -1.89999783 & -2.02059687 & -0.15010611 \\
\hline$S$ & 3.12166254 & -1.79528920 & -0.08687694 \\
\hline$N$ & 2.74835145 & 0.69032675 & 0.33828846 \\
\hline 0 & -1.62910607 & 2.48884972 & $-\odot .01274272$ \\
\hline C & $\odot .31034277$ & -1.90005600 & 1.31538248 \\
\hline $\mathrm{H}$ & 1.02099144 & -2.06029954 & 2.13124092 \\
\hline $\mathrm{H}$ & -0.68302447 & -1.79813042 & 1.75757255 \\
\hline $\mathrm{H}$ & $\odot .30611498$ & -2.81178275 & 0.70428414 \\
\hline C & 2.18871602 & 1.96613887 & ๑. 78937990 \\
\hline $\mathrm{H}$ & 2.89964150 & 2.42700360 & 1.47647692 \\
\hline $\mathrm{H}$ & 2.01875368 & 2.62765429 & -0.06363214 \\
\hline $\mathrm{H}$ & 1.24926236 & 1.79378227 & 1.31091800 \\
\hline $\mathrm{N}$ & -4.52585162 & -2.23314314 & -0.21598975 \\
\hline 0 & -4.23592734 & 2.64270482 & -0.10713071 \\
\hline C & -5.64298636 & 2.79994757 & -0.16784914 \\
\hline $\mathrm{H}$ & -6.04406961 & 2.36691654 & -1.09112984 \\
\hline $\mathrm{H}$ & -6.12489579 & 2.33427745 & 0.69912218 \\
\hline $\mathrm{H}$ & -5.83143206 & 3.87213276 & -0.15645798 \\
\hline 0 & -3.90992662 & -3.28045050 & -0.22587394 \\
\hline 0 & -5.73697756 & -2.14769573 & -0.25484034 \\
\hline $\mathrm{H}$ & -2.24071604 & 3.24078187 & $-\odot .04931607$ \\
\hline
\end{tabular}




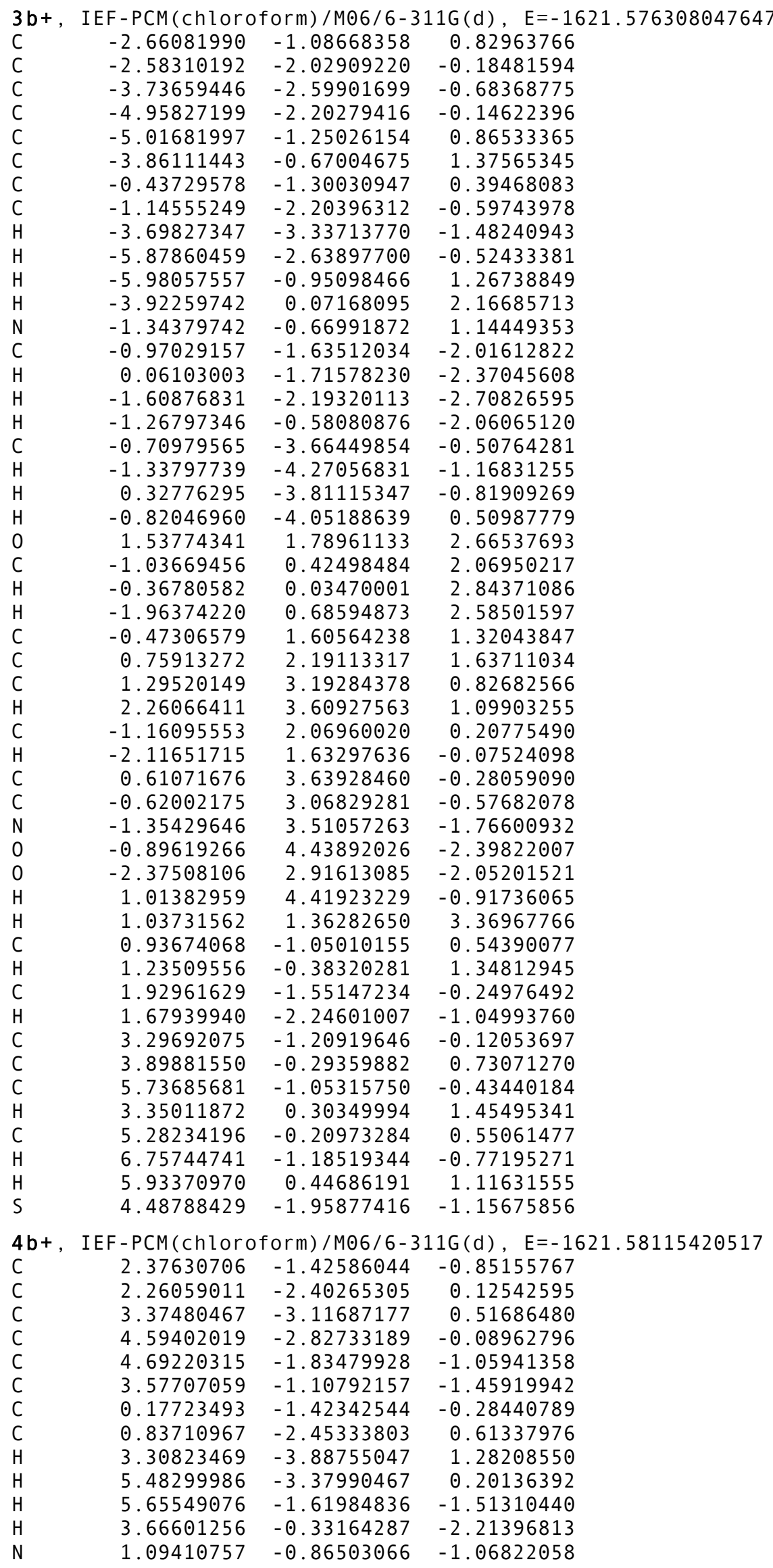




$\begin{array}{lrrr}\mathrm{C} & 0.79285469 & -1.96559223 & 2.06967918 \\ \mathrm{H} & -0.22379691 & -1.95791023 & 2.47247703 \\ \mathrm{H} & 1.39726345 & -2.63403196 & 2.69124494 \\ \mathrm{H} & 1.20834452 & -0.95483062 & 2.16006515 \\ \mathrm{C} & 0.24669090 & -3.85393248 & 0.45738782 \\ \mathrm{H} & 0.84001667 & -4.56248520 & 1.04425159 \\ \mathrm{H} & -0.78447269 & -3.91527046 & 0.81498268 \\ \mathrm{H} & 0.26741389 & -4.18069635 & -0.58691277 \\ \mathrm{O} & -1.32627248 & 1.96893522 & -2.37262407 \\ \mathrm{C} & 0.84653166 & 0.26921389 & -1.96162822 \\ \mathrm{H} & -0.01267799 & 0.04242698 & -2.59595498 \\ \mathrm{H} & 1.70471433 & 0.34732186 & -2.63373196 \\ \mathrm{C} & 0.64772274 & 1.54055539 & -1.18181553 \\ \mathrm{C} & -0.44947382 & 2.37403306 & -1.43460043 \\ \mathrm{C} & -0.62045976 & 3.55746860 & -0.71696772 \\ \mathrm{H} & -1.47709742 & 4.19434335 & -0.92641963 \\ \mathrm{C} & 1.54551039 & 1.90127625 & -0.19285592 \\ \mathrm{H} & 2.40239905 & 1.27306350 & 0.04001998 \\ \mathrm{C} & 0.28417789 & 3.91422546 & 0.26073290 \\ \mathrm{C} & 1.35658547 & 3.07372979 & 0.51807280 \\ \mathrm{~N} & 2.31082409 & 3.42724465 & 1.57193538 \\ \mathrm{O} & 2.14725115 & 4.48183123 & 2.15000015 \\ \mathrm{O} & 3.20756773 & 2.64207296 & 1.80884129 \\ \mathrm{H} & 0.16492193 & 4.82887310 & 0.83119967 \\ \mathrm{H} & -2.01007921 & 2.63385996 & -2.52255301 \\ \mathrm{C} & -1.17250754 & -1.01714960 & -0.33190987 \\ \mathrm{H} & -1.43139844 & -0.26293090 & -1.07100758 \\ \mathrm{C} & -2.16987314 & -1.49022041 & 0.46206982 \\ \mathrm{H} & -1.95080923 & -2.24727112 & 1.21273052 \\ \mathrm{C} & -3.53508761 & -1.06748458 & 0.40862584 \\ \mathrm{C} & -4.04525341 & -0.09981496 & -0.43772883 \\ \mathrm{C} & -5.77202670 & -1.07615558 & 1.02639559 \\ \mathrm{H} & -3.51696261 & 0.48943481 & -1.17862253 \\ \mathrm{H} & -6.71436934 & -1.29975149 & 1.50905741 \\ \mathrm{~S} & -5.71412675 & 0.12782495 & -0.21671791 \\ \mathrm{C} & -4.55302221 & -1.61890036 & 1.25072751 \\ \mathrm{H} & -4.36327228 & -2.39292406 & 1.98864621\end{array}$

$5 \mathrm{~b}+$, IEF-PCM (chloroform) /M06/6-311G(d) , E=-1817.942851144821

$\begin{array}{llll}\mathrm{C} & -5.18881434 & -0.89614172 & -1.10657665 \\ \mathrm{C} & -5.05466539 & -2.15676631 & -0.54456283 \\ \mathrm{C} & -6.17604503 & -2.92415607 & -0.30315190 \\ \mathrm{C} & -7.42421964 & -2.40100423 & -0.63155680 \\ \mathrm{C} & -7.53951461 & -1.12992464 & -1.18244162 \\ \mathrm{C} & -6.41635796 & -0.34772789 & -1.42963955 \\ \mathrm{C} & -2.94588306 & -1.16126063 & -0.78974175 \\ \mathrm{C} & -3.59920786 & -2.42885423 & -0.26172692 \\ \mathrm{H} & -6.09417908 & -3.91494176 & 0.13956595 \\ \mathrm{H} & -8.31909234 & -2.98935805 & -0.44903385 \\ \mathrm{H} & -8.52257826 & -0.73496477 & -1.42295055 \\ \mathrm{H} & -6.52084989 & 0.64815380 & -1.85160841 \\ \mathrm{~N} & -3.89931076 & -0.33348889 & -1.24409860 \\ \mathrm{C} & -3.12910900 & -3.68321083 & -0.99929669 \\ \mathrm{H} & -2.07940434 & -3.91583876 & -0.80100175 \\ \mathrm{H} & -3.72410882 & -4.54165056 & -0.67089679 \\ \mathrm{H} & -3.25856197 & -3.57942286 & -2.08153034 \\ \mathrm{C} & -3.39201592 & -2.54097819 & 1.25456261 \\ \mathrm{H} & -3.98426273 & -3.37676493 & 1.64167049 \\ \mathrm{H} & -2.34585126 & -2.72218714 & 1.51649552 \\ \mathrm{H} & -3.72131981 & -1.62998948 & 1.76791644 \\ \mathrm{O} & -1.47645153 & 3.01467004 & -1.88948540 \\ \mathrm{C} & -3.65463284 & 1.01886868 & -1.72840845 \\ \mathrm{H} & -4.57099155 & 1.36496056 & -2.21480430 \\ \mathrm{H} & -2.90578908 & 0.95093069 & -2.52628419 \\ \mathrm{C} & -3.23439598 & 1.93973838 & -0.60989781 \\ \mathrm{C} & -2.18463931 & 2.85731309 & -0.75368476\end{array}$




\begin{tabular}{|c|c|c|c|}
\hline C & -1.77092043 & 3.63442785 & ๑. 32985189 \\
\hline $\mathrm{H}$ & -0.94614269 & 4.32462963 & ๑. 17870194 \\
\hline C & -3.86540677 & 1.84411141 & 0.62183380 \\
\hline $\mathrm{H}$ & -4.68193651 & 1.14339164 & $\odot .77946875$ \\
\hline C & -2.39743423 & 3.52602188 & 1.54995851 \\
\hline C & -3.44683190 & 2.62579717 & 1.68113247 \\
\hline N & -4.11174069 & 2.47636121 & 2.97708205 \\
\hline 0 & -3.75748981 & 3.20636298 & 3.87992377 \\
\hline 0 & -4.97553642 & 1.62647148 & 3.07693703 \\
\hline $\mathrm{H}$ & -2.08658633 & 4.12414669 & 2.39947123 \\
\hline $\mathrm{H}$ & -1.92897539 & 2.66732116 & -2.66559115 \\
\hline C & -1.59620749 & -0.80356674 & -0.80540874 \\
\hline $\mathrm{H}$ & -1.35295457 & ๑. 17431294 & -1.21289261 \\
\hline C & $-\odot .56004682$ & -1.57230850 & -0.33012789 \\
\hline $\mathrm{H}$ & -0.78491775 & -2.55854419 & $\odot .07341098$ \\
\hline C & $\odot .80797674$ & -1.21607562 & -0.29425190 \\
\hline C & 1.30852659 & 0.03582399 & -0.72190078 \\
\hline C & 1.74466844 & -2.14583131 & ๑.20850494 \\
\hline C & 2.64106116 & 0.32994989 & -0.66079479 \\
\hline $\mathrm{H}$ & 0.63184380 & $\odot .80238074$ & -1.09317166 \\
\hline C & 3.08120902 & -1.86175741 & 0.28374991 \\
\hline $\mathrm{H}$ & 1.39164555 & -3.12285601 & $\odot .53432083$ \\
\hline C & 3.57061186 & -0.61066681 & -0.15201695 \\
\hline H & 2.99092205 & 1.30761159 & -0.97767770 \\
\hline $\mathrm{H}$ & 3.77317180 & -2.60761317 & 0.66210879 \\
\hline $\mathrm{N}$ & 4.90262744 & -0.30864890 & -0.07981798 \\
\hline C & 5.79964815 & -1.05604766 & $\odot .74602777$ \\
\hline C & 6.92556181 & -1.64125447 & $\odot .17850294$ \\
\hline C & 5.56939822 & -1.15742163 & 2.11423433 \\
\hline C & 7.81613950 & -2.33730926 & ๑. 98173768 \\
\hline $\mathrm{H}$ & 7.09782671 & -1.54532851 & -0.89148672 \\
\hline C & 6.45989595 & -1.86198341 & 2.90978407 \\
\hline $\mathrm{H}$ & 4.69437451 & -0.67802878 & 2.54853019 \\
\hline C & 7.58395161 & -2.45211822 & 2.34614326 \\
\hline H & 8.69568724 & -2.79597012 & 0.53733883 \\
\hline $\mathrm{H}$ & 6.28086701 & -1.93877539 & 3.97918873 \\
\hline H & 8.28383037 & -2.99820905 & 2.97324906 \\
\hline C & 5.46916225 & ๑.77386176 & -0.82309074 \\
\hline C & 5.37098330 & ๑.79516174 & -2.21048530 \\
\hline C & 6.15275103 & 1.78109044 & -0.15201495 \\
\hline C & 5.94359113 & 1.83767442 & -2.92334107 \\
\hline $\mathrm{H}$ & 4.84988946 & $-\odot . \odot 1 \odot 707 \odot \odot$ & -2.72354814 \\
\hline C & 6.72993190 & 2.81609410 & -0.87206072 \\
\hline $\mathrm{H}$ & 6.22894501 & 1.74624544 & ๑.93275670 \\
\hline C & 6.62377492 & 2.84856510 & -2.25653428 \\
\hline 11 & 5.86915211 & 1.85280141 & -4.00769873 \\
\hline 11 & 7. 26284764 & 3.60465185 & -0.34703089 \\
\hline $\mathrm{H}$ & 7.07730173 & 3.66121484 & -2.8180291 \\
\hline
\end{tabular}

$6 \mathrm{~b}+$, IEF-PCM (chloroform) /M06/6-311G(d), E=-1223.416772490666

$\begin{array}{lrrr}\mathrm{C} & 0.37629075 & 2.05204124 & 0.24784053 \\ \mathrm{C} & 1.23677373 & 2.30184492 & -0.80775596 \\ \mathrm{C} & 1.18217376 & 3.51999762 & -1.45648185 \\ \mathrm{C} & 0.25504875 & 4.45829381 & -1.01214061 \\ \mathrm{C} & -0.59753099 & 4.18354165 & 0.05367760 \\ \mathrm{C} & -0.55445336 & 2.96061427 & 0.71289482 \\ \mathrm{C} & 1.61339504 & 0.17428106 & 0.05824461 \\ \mathrm{C} & 2.10026292 & 1.09393050 & -1.03287501 \\ \mathrm{H} & 1.84480418 & 3.74639838 & -2.28911217 \\ \mathrm{H} & 0.19526298 & 5.42519881 & -1.50405162 \\ \mathrm{H} & -1.30936545 & 4.93705036 & 0.37806701 \\ \mathrm{H} & -1.21505108 & 2.74434887 & 1.54902328 \\ \mathrm{~N} & 0.65097233 & 0.73919730 & 0.73586098 \\ \mathrm{C} & 3.58842263 & 1.39054721 & -0.82950835 \\ \mathrm{H} & 4.18661859 & 0.48033243 & -0.93854806 \\ \mathrm{H} & 3.91784872 & 2.11096441 & -1.58465404\end{array}$




\begin{tabular}{|c|c|c|c|}
\hline $\mathrm{H}$ & 3.78069235 & 1.82143398 & ๑. 15927501 \\
\hline C & 1.84535291 & 0.48442042 & -2.41761211 \\
\hline $\mathrm{H}$ & 2.08933980 & 1.23425170 & -3.17681695 \\
\hline $\mathrm{H}$ & 2.48034335 & $-\odot .38748666$ & -2.59666309 \\
\hline $\mathrm{H}$ & $\odot .79419114$ & ๑. 20198122 & -2.55230665 \\
\hline 0 & -2.60252200 & 1.11294435 & 2.76945302 \\
\hline C & -0.19712010 & $\odot .10707300$ & 1.75799496 \\
\hline $\mathrm{H}$ & -0.27770371 & 0.79051162 & 2.60547090 \\
\hline $\mathrm{H}$ & 0.31187880 & -0.79603917 & 2.10249853 \\
\hline C & -1.53871926 & -0.21387860 & 1.16072365 \\
\hline C & -2.72129063 & 0.30101822 & 1.70763964 \\
\hline C & -3.95618951 & $-\odot .02 \odot 78348$ & 1.14210698 \\
\hline $\mathrm{H}$ & -4.86824703 & $\odot .38309968$ & 1.57610307 \\
\hline C & -1.61605023 & -1.02926127 & 0.04729491 \\
\hline $\mathrm{H}$ & -0.71991997 & -1.44952453 & -0.40288369 \\
\hline C & -4.02315901 & -0.83657699 & 0.03230597 \\
\hline C & -2.84513351 & -1.32938793 & -0.50993379 \\
\hline $\mathrm{N}$ & -2.88942876 & -2.17674858 & -1.70211351 \\
\hline 0 & -3.97907168 & -2.46983416 & -2.14714502 \\
\hline 0 & -1.82919824 & -2.53419875 & -2.18017117 \\
\hline $\mathrm{H}$ & -4.97559100 & -1.08853827 & -0.42143483 \\
\hline $\mathrm{H}$ & -3.46474902 & 1.40892222 & 3.08773503 \\
\hline C & 2.18695282 & -1.12821386 & 0.38811649 \\
\hline C & 2.09889379 & -2.21120294 & -0.48819359 \\
\hline C & 2.87848037 & -1.25792873 & 1.59631549 \\
\hline C & 2.68892112 & -3.41760484 & $-\odot .14523008$ \\
\hline $\mathrm{H}$ & 1.54172001 & -2.12891669 & -1.41814239 \\
\hline $\mathrm{C}$ & 3.48120915 & -2.46226252 & 1.91837105 \\
\hline $\mathrm{H}$ & 2.96371769 & -0.40385689 & 2.26591596 \\
\hline C & 3.38348847 & -3.54179542 & 1.05039293 \\
\hline $\mathrm{H}$ & 2.60323275 & -4.26571804 & -0.81833523 \\
\hline $\mathrm{H}$ & 4.02876720 & -2.55725634 & 2.85183467 \\
\hline $\mathrm{H}$ & 3.85162239 & -4.48783363 & 1.30856870 \\
\hline $7 b+$ & IEF-PCM (chloro & orm) / M०6/6-3 & $11 G(d), \quad E=-992.4899481334312$ \\
\hline & -1.74666267 & $\odot .69254867$ & $-\odot .08475128$ \\
\hline C & -2.66225856 & -0.22494576 & $\odot .41123954$ \\
\hline C & -3.43147595 & $\odot .10815876$ & 1.50795744 \\
\hline C & -3.25479522 & 1.36858858 & 2.07147391 \\
\hline C & -2.33225312 & 2.27292049 & 1.55133360 \\
\hline C & -1.54847334 & 1.95017751 & 0.44960128 \\
\hline C & -1.57408827 & -1.09224658 & -1.42364890 \\
\hline C & -2.59418969 & -1.48008685 & -0.41374160 \\
\hline $\mathrm{H}$ & -4.15507592 & -0.59116770 & 1.92168647 \\
\hline $\mathrm{H}$ & -3.84931663 & 1.65513243 & 2.93454452 \\
\hline $\mathrm{H}$ & -2.22165258 & 3.24949959 & 2.01365201 \\
\hline $\mathrm{H}$ & -0.83021383 & 2.65462576 & 0.03711385 \\
\hline $\mathrm{N}$ & -1.10318298 & $\odot .09195040$ & -1.21277006 \\
\hline C & -3.92207932 & -1.85897934 & -1.07042917 \\
\hline $\mathrm{H}$ & -3.80191947 & -2.73444295 & -1.71615166 \\
\hline $\mathrm{H}$ & -4.64907483 & -2.10970285 & -0.29180599 \\
\hline $\mathrm{H}$ & -4.32899627 & -1.03545143 & -1.66457488 \\
\hline C & -2.02763665 & -2.65582243 & 0.40184199 \\
\hline $\mathrm{H}$ & -2.72430509 & -2.88419642 & 1. 21464577 \\
\hline $\mathrm{H}$ & -1.91944788 & -3.54865332 & -0.22127212 \\
\hline $\mathrm{H}$ & -1.05521427 & -2.41277303 & $\odot .84453845$ \\
\hline 0 & 1.25936899 & 3.06220156 & -1.05470248 \\
\hline C & 0.02365492 & $\odot .71597099$ & -1.92486191 \\
\hline $\mathrm{H}$ & -0.27665142 & 1.72572498 & -2.21276267 \\
\hline $\mathrm{H}$ & $\odot .18457261$ & $\odot .13818262$ & -2.83937924 \\
\hline C & 1.24105057 & $\odot .72058714$ & -1.04466012 \\
\hline C & 1.83410875 & 1.92365930 & -0.63712650 \\
\hline C & 2.96350601 & 1.90947106 & $\odot .18187237$ \\
\hline $\mathrm{H}$ & 3.41406225 & 2.84931032 & $\odot .49281512$ \\
\hline C & 1.78798383 & -0.47537836 & -0.61639960 \\
\hline $\mathrm{H}$ & 1.36349045 & -1.42956463 & $-\odot .92084074$ \\
\hline
\end{tabular}




$\begin{array}{rrrr}\mathrm{C} & 3.49955403 & 0.71239420 & 0.60642452 \\ \mathrm{C} & 2.90036425 & -0.47234737 & 0.20470390 \\ \mathrm{~N} & 3.44838181 & -1.75117070 & 0.66132969 \\ \mathrm{O} & 4.45489104 & -1.72394202 & 1.33781717 \\ \mathrm{O} & 2.85804723 & -2.76412494 & 0.33990226 \\ \mathrm{H} & 4.37324189 & 0.68522113 & 1.24852523 \\ \mathrm{H} & 1.74996662 & 3.83848194 & -0.75576684 \\ \mathrm{H} & -1.20617322 & -1.71678115 & -2.23399699\end{array}$

$8 b+, \quad I E F-P C M(c h l o r$ oform) /M06/6-311G(d), E=-1434.688094554862

\begin{tabular}{|c|c|c|c|}
\hline & -3.41754706 & -1.16451786 & ๑.78195093 \\
\hline C & -3.30889674 & -2.15832677 & -0.17 \\
\hline C & -4.44516446 & -2.76816166 & -0.67020110 \\
\hline C & -5.68452205 & -2.35897452 & -0.18416391 \\
\hline C & -5.77470413 & -1.35510425 & ๑. 77286516 \\
\hline C & -4.63580955 & -0.73557440 & 1.27650038 \\
\hline C & -1.17820896 & -1.36567512 & ๑. 39596641 \\
\hline C & -1.85773270 & -2.35778186 & -0.53535892 \\
\hline $\mathrm{H}$ & -4.37898128 & -3.54916791 & -1.42538559 \\
\hline $\mathrm{H}$ & -6.59114491 & -2.82523601 & -0.5597 \\
\hline $\mathrm{H}$ & -6.75008518 & -1.04441753 & 1.13706010 \\
\hline $\mathrm{H}$ & -4.72334537 & ๑.๑4970711 & 2.02224254 \\
\hline N & -2.11673223 & -0.71504570 & 1.10440409 \\
\hline C & -1.63370283 & -1.94733053 & -1.99629406 \\
\hline $\mathrm{H}$ & -0.59141316 & -2.06838438 & -2.30478190 \\
\hline $\mathrm{H}$ & -2.25250860 & -2.57032362 & -2.65050233 \\
\hline $\mathrm{H}$ & -1.92018161 & $-\odot .90160134$ & -2.15910377 \\
\hline C & -1.43080871 & -3.80219563 & -0.27146520 \\
\hline $\mathrm{H}$ & -2.03321788 & -4.47785284 & -0.88810514 \\
\hline $\mathrm{H}$ & $-\odot .38088002$ & -3.98066725 & -0.51925345 \\
\hline $\mathrm{H}$ & -1.58708062 & -4.07537525 & $\odot .77727083$ \\
\hline 0 & 0.66453674 & 1.88930092 & 2.59605072 \\
\hline C & -1.84151306 & ๑.39718984 & $2.0 \odot 871275$ \\
\hline $\mathrm{H}$ & -1.14313244 & ๑.03930071 & 2.77362017 \\
\hline $\mathrm{H}$ & -2.76788917 & 0.62442450 & 2.54225031 \\
\hline C & -1.33811908 & 1.60246938 & 1.25485427 \\
\hline C & -0.14039014 & 2.25437789 & 1.57515319 \\
\hline C & $\odot .33146477$ & 3.30046950 & ๑. 78094178 \\
\hline $\mathrm{H}$ & 1.27162429 & 3.76914687 & 1.05692153 \\
\hline C & -2.05484921 & 2.04138204 & $\odot .15044618$ \\
\hline $\mathrm{H}$ & -2.98473786 & 1.55513887 & -0.13544139 \\
\hline C & -0.38468741 & 3.72599586 & -0.31416837 \\
\hline C & -1.58044900 & 3.08788032 & -0.61611025 \\
\hline $\mathrm{N}$ & -2.35220161 & 3.51726420 & -1.78524263 \\
\hline 0 & -1.93432367 & 4.46269860 & -2.42138867 \\
\hline 0 & -3.36393827 & 2.89935526 & -2.05242479 \\
\hline $\mathrm{H}$ & -0.03316340 & 4.54222524 & -0.93562269 \\
\hline $\mathrm{H}$ & ๑. 20352351 & 1.38025618 & 3.27185811 \\
\hline C & $\odot .17625060$ & -1.06406304 & ๑.52469662 \\
\hline $\mathrm{H}$ & $\odot .44064316$ & -0.28803031 & 1.23669417 \\
\hline C & 1.20322390 & -1.63310336 & -0.19565083 \\
\hline $\mathrm{H}$ & ๑.97162586 & -2.43723099 & -0.89327566 \\
\hline C & 2.56453272 & -1.26625652 & -0.14101720 \\
\hline C & 3.06177325 & -0.19473919 & 0.64120307 \\
\hline C & 3.50276646 & -1.97579052 & -0.92541813 \\
\hline C & 4.38534979 & ๑. 13688055 & 0.63947822 \\
\hline $\mathrm{H}$ & 2.38843720 & $\odot .39670071$ & 1.25923319 \\
\hline C & 4.83222083 & -1.65960116 & -0.93543721 \\
\hline $\mathrm{H}$ & 3.15174316 & -2.80149402 & -1.54219335 \\
\hline C & 5.32480403 & -0.58334246 & -0.15148670 \\
\hline $\mathrm{H}$ & 4.71563425 & ๑.96876411 & 1.25167488 \\
\hline $\mathrm{H}$ & 5.50621919 & -2.23877523 & -1.55655079 \\
\hline $\mathrm{N}$ & 6.63012071 & -0.24919516 & -0.15878245 \\
\hline C & 7.57481140 & $-\odot .99479357$ & -0.97156812 \\
\hline $\mathrm{H}$ & 7.61135335 & -2.05006191 & -0.67769121 \\
\hline $\mathrm{H}$ & 7. 31893744 & -0.93879355 & -2.03594398 \\
\hline
\end{tabular}




$\begin{array}{rrrr}\mathrm{H} & 8.57099869 & -0.57446480 & -0.84204631 \\ \mathrm{C} & 7.11260874 & 0.86225809 & 0.64193761 \\ \mathrm{H} & 6.61700717 & 1.79974629 & 0.36546372 \\ \mathrm{H} & 6.95367762 & 0.68625827 & 1.71222287 \\ \mathrm{H} & 8.18162163 & 0.98678014 & 0.47637514\end{array}$

$9 \mathrm{~b}+, \quad$ IEF-PCM (chloroform) /M06/6-311G(d), E=-1505.241517064337

$\begin{array}{llll}C & -3.28291008 & -1.28387612 & -0.81747111\end{array}$

0.15765678

C $\quad-4.28762441-2.94157913 \quad 0.58666102$

C $\quad-5.51457431-2.61476301 \quad 0.01668166$

$\begin{array}{llll}\text { C } & -5.61346511 & -1.62174281 & -0.95364537\end{array}$

C $\quad-4.49095484-0.93014383-1.39071710$

C $\quad-1.07021893-1.35822542-0.33020267$

$\begin{array}{llll}C & -1.73089123 & -2.35138466 & 0.60326422\end{array}$

$\mathrm{H} \quad-4.22096703-3.71249138 \quad 1.35137228$

$\begin{array}{llll}\mathrm{H} & -6.41056813 & -3.13986299 & 0.33547344\end{array}$

$\mathrm{H} \quad-6.58327632-1.38080175-1.37909065$

$\mathrm{H} \quad-4.58012428 \quad-0.15711497 \quad-2.14834311$

$\begin{array}{llll}\mathrm{N} & -1.98872340 & -0.76537560 & -1.07521637\end{array}$

$\begin{array}{llll}\text { C } & -1.18179660 & -3.76961209 & 0.46222400\end{array}$

$\begin{array}{llll}\mathrm{H} & -0.13976495 & -3.84586297 & 0.78374198\end{array}$

$\begin{array}{llll}\mathrm{H} & -1.77064316 & -4.44689727 & 1.08869899\end{array}$

$\begin{array}{llll}\mathrm{H} & -1.24879450 & -4.12235363 & -0.57142412\end{array}$

$\begin{array}{llll}\text { C } & -1.63305927 & -1.83392818 & 2.04974562\end{array}$

$\mathrm{H} \quad-2.25616202 \quad-2.46174212 \quad 2.69437575$

$\mathrm{H}-\begin{array}{llll}-0.61055573 & -1.86888508 & 2.43474505\end{array}$

$\mathrm{H} \quad-1.99741605 \quad-0.80291195 \quad 2.12783364$

$\begin{array}{llll}0 & 0.52802076 & 1.83970983 & -2.62521887\end{array}$

$\begin{array}{llll}\text { C } & -1.73387263 & 0.36671594 & -1.97575917\end{array}$

$\begin{array}{llll}\mathrm{H} & -2.65280551 & 0.54568596 & -2.53827916\end{array}$

$\begin{array}{llll}\mathrm{H} & -0.97899007 & 0.07676046 & -2.70904961\end{array}$

$\begin{array}{llll}\text { C } & -1.31941331 & 1.58998936 & -1.20566746\end{array}$

$\begin{array}{llll}\text { C } & -0.17904720 & 2.31123565 & -1.58150326\end{array}$

$\begin{array}{llll}C & 0.20717663 & 3.44640641 & -0.87023151\end{array}$

$\begin{array}{llll}\mathrm{H} & 1.09393645 & 3.99713227 & -1.17674585\end{array}$

C $\quad-2.04544563 \quad 2.01195368-0.10606752$

$\begin{array}{llll}\mathrm{H} & -2.93184112 & 1.47442413 & 0.22365209\end{array}$

$\begin{array}{llll}\text { C } & -0.52564820 & 3.86440957 & 0.22092270\end{array}$

$\begin{array}{llll}\mathrm{C} & -1.64245056 & 3.13390548 & 0.59727294\end{array}$

$\begin{array}{llll}\mathrm{N} & -2.41008638 & 3.54443673 & 1.77569806\end{array}$

$\begin{array}{lllll}0 & -3.33145571 & 2.83198781 & 2.12351206\end{array}$

$\begin{array}{llll}0 & -2.07794826 & 4.56658671 & 2.33836386\end{array}$

$\mathrm{H} \quad-0.23532343 \quad 4.74164128 \quad 0.78876682$

$\begin{array}{llll}\mathrm{H} & 1.26044792 & 2.42829866 & -2.84764229\end{array}$

C $\quad 0.30527063-1.01939990-0.45026681$

$\begin{array}{llll}\mathrm{H} & 0.57052219 & -0.35238214 & -1.26656565\end{array}$

$\begin{array}{llll}\text { C } & 1.28872331 & -1.47386998 & 0.35805359\end{array}$

$\begin{array}{llll}\mathrm{H} & 1.04147736 & -2.14863941 & 1.17525365\end{array}$

$\begin{array}{llll}\text { C } & 2.69218193 & -1.13185157 & 0.25629843\end{array}$

$\begin{array}{llll}\text { C } & 3.58441929 & -1.73840814 & 1.14991845\end{array}$

$\begin{array}{llll}C & 3.18898493 & -0.22277013 & -0.69052824\end{array}$

C $\quad 4.93731531-1.46032369 \quad 1.10261011$

$\begin{array}{llll}\mathrm{H} & 3.20785174 & -2.44069222 & 1.88944088\end{array}$

$\begin{array}{llll}\text { C } & 4.53671764 & 0.06030676 & -0.74809747\end{array}$

$\begin{array}{llll}\mathrm{H} & 2.52105555 & 0.27429217 & -1.38962501\end{array}$

$\begin{array}{llll}\text { C } & 5.38949502 & -0.56493026 & 0.15041979\end{array}$

$\begin{array}{llll}\mathrm{H} & 5.63742450 & -1.92433293 & 1.78822089\end{array}$

$\begin{array}{llll}\mathrm{H} & 4.93895394 & 0.75812737 & -1.47391658\end{array}$

$\begin{array}{llll}\mathrm{N} & 6.83381041 & -0.25846917 & 0.08908487\end{array}$

$\begin{array}{llll}0 & 7.20261640 & 0.52394071 & -0.76008067\end{array}$

$\begin{array}{llll}0 & 7.55601974 & -0.80816592 & 0.89126247\end{array}$

$10 \mathrm{~b}+$, IEF-PCM (chloroform) /M06/6-311G(d), E=-1414.068481771956

$\begin{array}{llll}\text { C } & 2.96413062 & -1.27976746 & 0.83096597 \\ \text { C } & 2.86773600 & -2.25758317 & -0.14683042 \\ \text { C } & 4.00656881 & -2.90125636 & -0.58721424\end{array}$




\begin{tabular}{|c|c|c|c|}
\hline C & 5.22899756 & -2.53734799 & -0.03014382 \\
\hline C & 5.30630739 & -1.54441047 & ๑.94169457 \\
\hline C & 4.16652381 & -0.89081130 & 1.39312204 \\
\hline C & $\odot .75049544$ & -1.39482915 & 0.33671551 \\
\hline C & 1.43410895 & -2.38518935 & -0.58614853 \\
\hline $\mathrm{H}$ & 3.95694592 & -3.67039644 & -1.35509407 \\
\hline $\mathrm{H}$ & 6.13791513 & -3.03090554 & -0.36237903 \\
\hline $\mathrm{H}$ & 6.27242944 & -1.27121931 & 1.35592638 \\
\hline $\mathrm{H}$ & 4.24086286 & -0.11458269 & 2.14928608 \\
\hline $\mathrm{N}$ & 1.65858513 & -0.79349837 & 1.09368425 \\
\hline C & $\odot .92847088$ & -3.81821409 & -0.42131421 \\
\hline $\mathrm{H}$ & -0.09906674 & -3.94368675 & -0.77131148 \\
\hline $\mathrm{H}$ & 1.55793756 & -4.49044312 & -1.01280764 \\
\hline $\mathrm{H}$ & ๑.97927466 & -4.14229405 & 0.62249009 \\
\hline C & 1.31448852 & -1.89210176 & -2.03783744 \\
\hline $\mathrm{H}$ & 1.93774920 & -2.52012391 & -2.68210069 \\
\hline $\mathrm{H}$ & 0.28726716 & -1.94227829 & -2.40887322 \\
\hline $\mathrm{H}$ & 1.66551779 & -0.85805757 & -2.13400359 \\
\hline 0 & -1.09468649 & 1.89580923 & 2.37993931 \\
\hline C & 1.38304791 & ๑. 31776294 & 2.01357141 \\
\hline $\mathrm{H}$ & 2.27573896 & $\odot .45975110$ & 2.62637512 \\
\hline $\mathrm{H}$ & 0.60162998 & -0.01328409 & 2.70235076 \\
\hline C & 1.02475826 & 1. 56462091 & 1.24917863 \\
\hline C & -0.17460504 & 2.25870163 & 1.45704033 \\
\hline C & -0.51760165 & 3.33601822 & $\odot .64096234$ \\
\hline $\mathrm{H}$ & -1.46324059 & 3.83773681 & 0.82403366 \\
\hline C & 1.88322883 & 2.00321918 & $\odot .25008957$ \\
\hline $\mathrm{H}$ & 2.82755514 & 1.49680543 & ๑.06213294 \\
\hline C & ๑. 33231917 & 3.75499370 & -0.35806940 \\
\hline C & 1.53390722 & 3.08308487 & -0.53617216 \\
\hline $\mathrm{N}$ & 2.45202133 & 3.51663819 & -1.59523339 \\
\hline 0 & 2.11922833 & 4.46657973 & -2.27215653 \\
\hline 0 & 3.48786924 & 2.89754242 & -1.73330147 \\
\hline $\mathrm{H}$ & $\odot .08321985$ & 4.59626868 & -0.99550812 \\
\hline $\mathrm{H}$ & -0.70379209 & 1.48015008 & 3.15702154 \\
\hline C & -0.62144121 & -1.04726814 & $\odot .41319543$ \\
\hline $\mathrm{H}$ & -0.89671810 & -0.28540554 & 1.13683319 \\
\hline C & -1.60697424 & -1.58395593 & -0.34648321 \\
\hline $\mathrm{H}$ & -1.36800723 & -2.37378511 & -1.05587893 \\
\hline C & -3.00033312 & -1.20776878 & -0.30817712 \\
\hline C & -3.90827240 & -1.97804227 & -1.04784071 \\
\hline C & -3.47516315 & -0.10601014 & 0.42809895 \\
\hline C & -5.25712607 & -1.67548596 & -1.03619206 \\
\hline $\mathrm{H}$ & -3.54337653 & -2.82201445 & -1.62919392 \\
\hline C & -4.81765696 & $\odot .19446578$ & 0.43157398 \\
\hline $\mathrm{H}$ & -2.79148397 & 0.52653973 & $\odot .99021081$ \\
\hline C & -5.71540619 & -0.59141498 & -0.29593114 \\
\hline $\mathrm{H}$ & -5.96176047 & -2.27904465 & -1.60506573 \\
\hline $\mathrm{H}$ & -5.20170353 & 1.04298521 & 0.99188253 \\
\hline C & -7.15810007 & -0.26639273 & -0.28506992 \\
\hline 0 & -7.63903026 & 0.64892079 & ๑. 32919337 \\
\hline $\mathrm{H}$ & $-7.7906419 \odot$ & -0.94561475 & -0.90158625 \\
\hline
\end{tabular}

$11 \mathrm{~b}+$, IEF-PCM (chloroform) /M06/6-311G(d), E=-1777.980797436603

$-4.46379624-2.46339103$

0.05304998

$-5.50197784-3.25465872$

0.50060480

$-6.80358530 \quad-2.90355786$

0.15062494

$-7.05418125$

$-1.77646530$

$-0.62462975$

$-6.01591761$

$-0.97283762$

$-2.47020503$

$-1.35991047$

$-1.08228357$

$-2.97847182$

$-2.55952899$

$-0.49457681$

๑. 28936689

$-5.31326692$

$-4.13029237$

1. 11891656

$-7.63531798$

$-3.51263062$

0.49372381

$-8.07803280$

$-1.51407041$

$-0.87599965$

$\mathrm{H}$

$-6.22108255$

$-0.08678597$

$-1.67713234$ 


\begin{tabular}{|c|c|c|c|}
\hline $\mathrm{N}$ & -3.50717662 & -0.73127871 & -1.05798058 \\
\hline C & -2.43068004 & -3.87706547 & -0.26426790 \\
\hline $\mathrm{H}$ & -1.35295547 & -3.97745243 & -0.10902796 \\
\hline $\mathrm{H}$ & -2.91865185 & -4.71477714 & ๑. 24490190 \\
\hline $\mathrm{H}$ & -2.63170796 & -3.97078343 & -1.33643847 \\
\hline C & -2.68579394 & -2.40348605 & 1.78654229 \\
\hline$\pi$ & -3.18988475 & -3.20336874 & 2.33886408 \\
\hline $\mathrm{H}$ & -1.61728536 & -2.46799403 & 2.01014121 \\
\hline $\mathrm{H}$ & -3.05684479 & -1.44609743 & 2.16904815 \\
\hline 0 & -2.28781210 & 2.75754991 & -2.84929488 \\
\hline C & -3.41100866 & ๑. 48794481 & -1.84717527 \\
\hline $\mathrm{H}$ & -4.31607430 & ๑. 56148178 & -2.45908703 \\
\hline $\mathrm{H}$ & -2.58790498 & ๑.38748985 & -2.56090299 \\
\hline C & -3.22642373 & 1.70987533 & -0.98947461 \\
\hline C & -2.63317996 & 2.84732988 & -1.55711739 \\
\hline C & -2.42323205 & 3.99340843 & -0.79221569 \\
\hline $\mathrm{H}$ & -1.95173523 & 4.86382508 & -1.24350251 \\
\hline C & -3.60926258 & 1. 74820431 & ๑. 33578687 \\
\hline $\mathrm{H}$ & -4.07759239 & ๑.89125965 & 0.81214568 \\
\hline C & -2.80790189 & 4.02313242 & 0.53270379 \\
\hline C & -3.39846266 & 2.89701186 & 1.08362057 \\
\hline $\mathrm{N}$ & -3.79905350 & 2.91010285 & 2.48881102 \\
\hline 0 & -3.64742856 & 3.94181245 & 3.11169577 \\
\hline 0 & -4.26034732 & 1.88664626 & 2.95818883 \\
\hline $\mathrm{H}$ & -2.65111596 & 4.90533507 & 1. 14371155 \\
\hline $\mathrm{H}$ & -1.84156428 & 3.55820760 & -3.15229676 \\
\hline C & -1.16325054 & -0.87257966 & -0.63936475 \\
\hline $\mathrm{H}$ & -1.02295260 & 0.03776199 & -1.21120652 \\
\hline C & -0.04599498 & -1.44519043 & $-\odot .09088396$ \\
\hline $\mathrm{H}$ & $-\odot .15545194$ & -2.36153907 & ๑.48823481 \\
\hline C & 1.29025649 & -0.98209962 & -0.17736893 \\
\hline C & 2.30104709 & -1.69556033 & 0.45289582 \\
\hline C & 1.63476235 & ๑.23965991 & -0.90295364 \\
\hline C & 3.63081657 & -1.29685249 & ๑.40902484 \\
\hline $\mathrm{H}$ & 2.04131519 & -2.59948798 & $1 . \odot \odot 48716 \odot$ \\
\hline C & $4.7 \odot \odot 52415$ & -1.97664322 & 1.03768459 \\
\hline C & 3.94966244 & -0.12580195 & -0.31 \\
\hline C & 5.97950864 & -1.52287940 & ๑. 95088262 \\
\hline $\mathrm{H}$ & 4.48452223 & -2.88555686 & 1.59545537 \\
\hline C & 5. 22969194 & $\odot .34867186$ & -0.41830383 \\
\hline C & 6.29405352 & -0.33635487 & ๑.21311492 \\
\hline $\mathrm{H}$ & 6.76800635 & -2.08733718 & 1.43510943 \\
\hline $\mathrm{H}$ & 5.38203490 & 1. 26647650 & -0.97436961 \\
\hline 0 & $\odot .88352165$ & $\odot .97749162$ & -1.47952042 \\
\hline 0 & 2.97148483 & ๑.58093177 & -0.92709664 \\
\hline $\mathrm{N}$ & 7.56265303 & ๑.10914396 & ๑. 12324895 \\
\hline C & 7.92625589 & 1. 24790451 & -0.71737072 \\
\hline $\mathrm{H}$ & 8.95842145 & 1.08499157 & -1.04776459 \\
\hline $\mathrm{H}$ & 7. 32161809 & 1.23385552 & -1.62976136 \\
\hline C & 8.66444961 & -0.50609280 & ๑.86165966 \\
\hline $\mathrm{H}$ & 9.40180330 & ๑. 28298789 & 1.04781159 \\
\hline $\mathrm{H}$ & 8. 31417671 & -0.81653268 & 1.85088627 \\
\hline C & 9.30228231 & -1.65637735 & ๑. 10888996 \\
\hline $\mathrm{H}$ & 10.14137600 & -2.06959818 & ๑.67679273 \\
\hline $\mathrm{H}$ & 8.58610564 & -2.46455403 & -0.07336997 \\
\hline $\mathrm{H}$ & 9.68615820 & -1.32632648 & -0.86252866 \\
\hline C & 7.80714990 & 2.57153699 & $\odot .01114300$ \\
\hline $\mathrm{H}$ & 8.11697282 & 3.39696366 & -0.63697575 \\
\hline $\mathrm{H}$ & 6.77876134 & 2.76343191 & $\odot .33458387$ \\
\hline $\mathrm{H}$ & 8.44435166 & 2.58848798 & $\odot .90206964$ \\
\hline
\end{tabular}

$12 \mathrm{~b}+$, IEF-PCM (chloroform) /M06/6-311G(d), E=-1625.148940755211

$\begin{array}{llll}C & -3.91818646 & -1.05658259 & -1.00267060 \\ C & -3.90716146 & -2.26623711 & -0.32795887 \\ C & -5.09382799 & -2.93110685 & -0.09372196 \\ C & -6.27825854 & -2.35252907 & -0.54219579\end{array}$




\begin{tabular}{|c|c|c|c|}
\hline C & -6.26900154 & -1.12906756 & -1.20393452 \\
\hline C & $-5.0794 \odot 10 \odot$ & -0.45171982 & -1.44670743 \\
\hline C & -1.72324732 & -1.43062844 & -0.52446679 \\
\hline$r$ & $-2.49080 \odot \odot 2$ & -2.61663597 & $\odot .04647798$ \\
\hline $\mathrm{H}$ & -5.11020198 & -3.88373547 & 0.43211583 \\
\hline $\mathrm{H}$ & -7.22364415 & -2.85871587 & -0.36731586 \\
\hline $\mathrm{H}$ & -7.20601313 & -0.69098973 & -1.53629239 \\
\hline H & -5.08022800 & $\odot .50926480$ & -1.95400823 \\
\hline N & -2.58481898 & $-\odot .59889876$ & -1.12125056 \\
\hline C & $-2.08104 \odot 18$ & -3.93823145 & -0.61036776 \\
\hline H & -1.06614758 & -4.24235433 & -0.34066187 \\
\hline $\mathrm{H}$ & -2.76034491 & -4.73042113 & -0.27871089 \\
\hline H & -2.14355415 & -3.87636247 & -1.70166133 \\
\hline 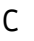 & -2.36069907 & -2.67733695 & 1.57291238 \\
\hline H & $-3.02407 \odot 81$ & -3.45730864 & 1.96092423 \\
\hline $\mathrm{H}$ & -1.34319547 & -2.91605585 & 1.89275426 \\
\hline $\mathrm{H}$ & -2.65148895 & -1.73020532 & 2.04056020 \\
\hline 0 & -1.91661124 & 3.19765174 & -2.64589896 \\
\hline C & -2.26419011 & 0.66543674 & -1.75963330 \\
\hline $\mathrm{H}$ & -2.97226583 & $\odot .81245468$ & -2.58239598 \\
\hline $\mathrm{H}$ & -1.28501049 & $\odot .58964477$ & -2.24337811 \\
\hline C & -2.31477009 & 1.82655228 & -0.80481068 \\
\hline C & -2.13058916 & 3.11722577 & -1.32423448 \\
\hline C & -2.17099315 & 4.23193533 & -0.49102581 \\
\hline $\mathrm{H}$ & -2.03025720 & 5.22391994 & -0.91504464 \\
\hline C & -2.53457800 & 1.68052434 & $\odot .54852479$ \\
\hline $\mathrm{H}$ & -2.69135994 & 0.70312272 & ๑. 99432261 \\
\hline C & -2.39002306 & 4.07607740 & 0.86351066 \\
\hline C & -2.56698499 & 2.79894890 & 1.36914546 \\
\hline $\mathrm{N}$ & -2.79432790 & 2.61656797 & 2.80227490 \\
\hline 0 & -2.83443488 & 3.60965858 & 3.49986762 \\
\hline 0 & -2.92812284 & 1.48044441 & 3.21593973 \\
\hline $\mathrm{H}$ & -2.42377205 & 4.93174006 & 1. 52876040 \\
\hline C & 4.73707013 & -1.47068742 & 0.51382780 \\
\hline $\mathrm{H}$ & -1.80862129 & 4.11434038 & -2.92913185 \\
\hline C & $-\odot .35139386$ & -1.15339354 & -0.44555882 \\
\hline $\mathrm{H}$ & -0.01894999 & $-\odot .2 \odot 217592$ & -0.85395666 \\
\hline C & $\odot .58297077$ & -1.98191522 & ๑. 11823095 \\
\hline $\mathrm{H}$ & $\odot .24852990$ & -2.93011685 & 0.53576479 \\
\hline C & 1.98244922 & -1.75352731 & ๑. 22584191 \\
\hline C & 2.77058991 & -2.74837092 & 0.86067966 \\
\hline C & 2.61426897 & -0.59449577 & -0.26712489 \\
\hline C & 4.12884637 & -2.62612696 & 1.01360460 \\
\hline $\mathrm{H}$ & 2.27244410 & -3.63828056 & 1. 24002651 \\
\hline C & 3.97318543 & -0.44964482 & -0.12622495 \\
\hline $\mathrm{H}$ & 2.03993220 & $\odot .18634293$ & -0.76141370 \\
\hline $\mathrm{H}$ & 4.69965115 & -3.40570266 & 1. 50935541 \\
\hline C & 4.90841207 & 0.58061677 & -0.51018780 \\
\hline C & 6.17567059 & 0.13752395 & -0.09175296 \\
\hline C & 4.78697611 & 1.80654529 & -1.15487855 \\
\hline $\mathrm{C}$ & 7.32507113 & 0.88563065 & $-\odot .30084988$ \\
\hline $\mathrm{C}$ & 5.92877168 & 2.56174399 & -1.36874646 \\
\hline $\mathrm{H}$ & 3.81519249 & 2.16812214 & -1.48538941 \\
\hline $\mathrm{C}$ & 7.17977516 & 2.10465617 & -0.94598163 \\
\hline $\mathrm{H}$ & 8.30167274 & 0.53794679 & ๑. $0264 \odot 199$ \\
\hline $\mathrm{H}$ & 5.85369268 & 3.52289061 & -1.87057926 \\
\hline $\mathrm{H}$ & 8.05959384 & 2.71704793 & -1.12621856 \\
\hline $\mathrm{N}$ & 6.04706761 & -1.10554557 & $\odot .52227779$ \\
\hline $\mathrm{C}$ & 7.15785120 & -1.84651027 & 1.07110958 \\
\hline $\mathrm{H}$ & 7.59554101 & -1.31648648 & 1.92332624 \\
\hline $\mathrm{H}$ & 6.82082329 & -2.82736489 & 1.40600745 \\
\hline H & 7.92939788 & -1.98948222 & $\odot .3084798$ \\
\hline
\end{tabular}


<smiles>O=[N+]([O-])c1ccc(O)cc1</smiles>

frag-A<smiles>O=[N+]([O-])c1ccc([O-])cc1</smiles>

frag-B

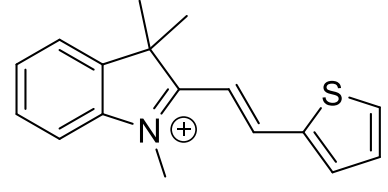

frag-C

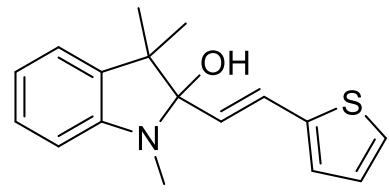

frag-D

frag-A, IEF-PCM(acetonitrile)/M066-311G(d), $E=-511.7895511491907$

C

C

C

C

C

C

$\mathrm{H}$

$\mathrm{H}$

$\mathrm{H}$

O

$\mathrm{H}$

O
$-0.00000470$

0.00000168

0.00000212

0.00000084

$-0.00000172$

$-0.00000352$

0.00000743

0.00000422

$-0.00000003$

$-0.00000395$

0.00000217

0.00000406

0.00000131

$-0.00000605$

0.00000524

frag-B, IEF-PCM (acetonitrile)/M066-311G(d), E=-511.3278614232449

$-2.19604708$

0.00000287

0.00000555

$-1.41398334$

1. 21901448

$-0.00000362$

$-0.05049880$

1.21687387

$-0.00001102$

0.65915874

$-0.00000292$

$-0.00000713$

$-0.05050130$

$-1.21687706$

$-0.00001271$

$-1.41398565$

$-1.21901517$

$-0.00000925$

$-1.96578797$

2. 15847034

0.51162877

2.14693792

$-0.00001334$

0.51163573

$-2.14693523$

$-0.00000800$

$-1.96579450$

$-2.15846825$

$-3.44215532$

$-0.00000013$

$-0.00001325$

$-0.00000428$

2.06936854

0.00000141

0.00001494

2.67219303

1. 07843273

0.00000569

2.67219763

$-1.07843148$

0.00000417

0.00000941

frag-C, IEF-PCM(acetonitrile)/M066-311G(d), E=-1110.996369725116

2.82404468

0.74132685

$-0.00039106$

2.70199659

$-0.64003089$

0.00035102

3. 83235428

$-1.43223776$

0.00159281

5.07807154

$-0.80968425$

0.00239861

5.18055661

0.57757794

0.00196317

4.04839749

1.38406091

0.00055767

0.58850382

0.35988265

1.24279899

$-1.01167943$

$-0.00110397$

3.75652231

$-2.51783820$

$-0.00053901$

5.98094674

$-1.41418600$

0.00206407

6.16209429

1.04353576

0.00348849

4.14364379

2.46545628

0.00284893

1. 52572978

1. 30232565

0.00059158

0.90057435

$-1.79910822$

$-0.00216502$

$-0.15230816$

$-2.09049564$

1.26811186

1.30655054 


$\begin{array}{lrrr}\mathrm{H} & 1.49852786 & -2.71577779 & 1.29435757 \\ \mathrm{H} & 1.13003340 & -1.21913603 & 2.16790476 \\ \mathrm{C} & 0.90233381 & -1.79908365 & -1.26974915 \\ \mathrm{H} & 1.50039530 & -2.71571013 & -1.29519540 \\ \mathrm{H} & -0.15047011 & -2.09053663 & -1.30965876 \\ \mathrm{H} & 1.13303059 & -1.21904981 & -2.16918695 \\ \mathrm{C} & 1.26854544 & 2.73471449 & -0.00317489 \\ \mathrm{H} & 0.69198376 & 3.01413963 & -0.88774190 \\ \mathrm{H} & 2.21528710 & 3.26949701 & -0.02331438 \\ \mathrm{C} & -0.78180164 & 0.68807547 & 0.00013076 \\ \mathrm{H} & -1.03500934 & 1.74572688 & 0.00263148 \\ \mathrm{C} & -1.79891498 & -0.22025978 & -0.00091649 \\ \mathrm{H} & -1.56238371 & -1.28240438 & -0.00304171 \\ \mathrm{C} & -3.18046103 & 0.10272683 & 0.00031394 \\ \mathrm{C} & -3.80374400 & 1.33895714 & 0.00266053 \\ \mathrm{C} & -5.64382310 & -0.04989781 & 0.00095928 \\ \mathrm{H} & -3.26259898 & 2.27982021 & 0.00401334 \\ \mathrm{C} & -5.20052688 & 1.24928029 & 0.00302125 \\ \mathrm{H} & -6.66877185 & -0.39929850 & 0.00072592 \\ \mathrm{H} & -5.86702578 & 2.10455974 & 0.00466887 \\ \mathrm{~S} & -4.36698632 & -1.18025104 & -0.00144520 \\ \mathrm{H} & 0.72433958 & 3.02170997 & 0.89945389\end{array}$

frag-D, IEF-PCM (acetonitrile)/M066-311G(d), $E=-1186.969857121705$

$\begin{array}{lrrr}\mathrm{C} & -2.74242818 & 0.74990844 & -0.18447411 \\ \mathrm{C} & -2.75052556 & -0.64494516 & -0.09068828 \\ \mathrm{C} & -3.93988165 & -1.33779601 & -0.06122174 \\ \mathrm{C} & -5.14377849 & -0.62726444 & -0.11197187 \\ \mathrm{C} & -5.12807729 & 0.75708504 & -0.19320009 \\ \mathrm{C} & -3.92762869 & 1.46924577 & -0.22466471 \\ \mathrm{C} & -0.57248058 & 0.16952855 & 0.32783876 \\ \mathrm{C} & -1.32336127 & -1.13466103 & -0.10189496 \\ \mathrm{H} & -3.94649081 & -2.42529075 & 0.00831938 \\ \mathrm{H} & -6.09159627 & -1.15941660 & -0.08097300 \\ \mathrm{H} & -6.06815690 & 1.30446808 & -0.22318945 \\ \mathrm{H} & -3.93158897 & 2.55565355 & -0.27520561 \\ \mathrm{~N} & -1.43374811 & 1.22130916 & -0.22608705 \\ \mathrm{C} & -0.95954856 & -1.53182248 & -1.53422539 \\ \mathrm{H} & 0.07687902 & -1.88407404 & -1.60392353 \\ \mathrm{H} & -1.61336875 & -2.34912501 & -1.86003941 \\ \mathrm{H} & -1.08962556 & -0.69962328 & -2.23464473 \\ \mathrm{C} & -1.04716293 & -2.30022203 & 0.82828299 \\ \mathrm{H} & -1.53593695 & -3.20522504 & 0.44822215 \\ \mathrm{H} & 0.02928657 & -2.50680168 & 0.88069981 \\ \mathrm{H} & -1.41041713 & -2.11323315 & 1.84125728 \\ \mathrm{C} & -1.16283706 & 2.59287279 & 0.12787829 \\ \mathrm{H} & -1.52005455 & 2.85146645 & 1.13839037 \\ \mathrm{H} & -1.64294746 & 3.27148646 & -0.58372854 \\ \mathrm{C} & 0.81753894 & 0.28292617 & -0.21697038 \\ \mathrm{H} & 0.88443787 & 0.58148531 & -1.26349283 \\ \mathrm{C} & 1.92205064 & 0.00875844 & 0.48055306 \\ \mathrm{H} & 1.81599798 & -0.28247178 & 1.52713193 \\ \mathrm{C} & 3.27256848 & 0.04886097 & -0.04622432 \\ \mathrm{C} & 3.71380913 & 0.19902122 & -1.33536729 \\ \mathrm{C} & 5.75066213 & 0.02554348 & -0.25203302 \\ \mathrm{H} & 3.04139644 & 0.30233270 & -2.18244549 \\ \mathrm{C} & 5.12306074 & 0.18814469 & -1.45117721 \\ \mathrm{H} & 6.81273916 & -0.02192565 & -0.04767852 \\ \mathrm{H} & 5.65435224 & 0.29110586 & -2.39222351\end{array}$




\begin{tabular}{|c|c|c|c|}
\hline S & 4.62380862 & -0.11696383 & 1.04167215 \\
\hline O & -0.58202014 & 0.19693392 & 1.74238408 \\
\hline $\mathrm{H}$ & -0.08013321 & 0.96633621 & 2.03859463 \\
\hline $\mathrm{H}$ & -0.08519160 & 2.77853170 & 0.07907297 \\
\hline $3 a$, & IEF-PCM (acetoni & trile)/M06/6- & $311 G(d), \quad-1621.160499103334$ \\
\hline $\mathrm{C}$ & 2.06652657 & -1.86390789 & 0.73182133 \\
\hline $\mathrm{C}$ & 1.75322998 & -2.79190606 & -0.26286597 \\
\hline $\mathrm{C}$ & 2.72416907 & -3.62747388 & -0.77036267 \\
\hline $\mathrm{C}$ & 4.03003041 & -3.52237852 & -0.28572634 \\
\hline $\mathrm{C}$ & 4.33368725 & -2.58994884 & 0.69553196 \\
\hline $\mathrm{C}$ & 3.35658930 & -1.74197578 & 1.21859781 \\
\hline $\mathrm{C}$ & -0.03592214 & -1.30282710 & 0.02321398 \\
\hline $\mathrm{C}$ & 0.27232743 & -2.71760484 & -0.55038249 \\
\hline $\mathrm{H}$ & 2.47980202 & -4.35534888 & -1.54308168 \\
\hline $\mathrm{H}$ & 4.81026305 & -4.16802866 & -0.68105696 \\
\hline $\mathrm{H}$ & 5.35422675 & -2.50801483 & 1.06306615 \\
\hline $\mathrm{H}$ & 3.61602585 & -1.01259125 & 1.98238477 \\
\hline $\mathrm{N}$ & 0.90344176 & -1.17337423 & 1.11491373 \\
\hline C & -0.44490993 & -3.79369037 & 0.26728540 \\
\hline $\mathrm{H}$ & -1.52839217 & -3.76587807 & 0.10120023 \\
\hline $\mathrm{H}$ & -0.08366105 & -4.78050115 & -0.04288233 \\
\hline $\mathrm{H}$ & -0.25503144 & -3.68952262 & 1.34106038 \\
\hline C & -0.10395054 & -2.86283948 & -2.01318507 \\
\hline $\mathrm{H}$ & 0.06300505 & -3.89633747 & -2.33750150 \\
\hline $\mathrm{H}$ & -1.16801980 & -2.64091583 & -2.16271655 \\
\hline $\mathrm{H}$ & 0.48006154 & -2.20368155 & -2.65903360 \\
\hline O & 0.30488904 & -0.36912347 & -1.04145302 \\
\hline $\mathrm{C}$ & 0.97380425 & 0.15414552 & 1.69026736 \\
\hline $\mathrm{H}$ & 1.80258442 & 0.20182980 & 2.40321903 \\
\hline $\mathrm{H}$ & 0.06001533 & 0.33511399 & 2.27280554 \\
\hline C & 1.12098583 & 1.18668555 & 0.61211925 \\
\hline $\mathrm{C}$ & 0.75852431 & 0.84842613 & -0.69962128 \\
\hline $\mathrm{C}$ & 0.87316885 & 1.78236097 & -1.73310358 \\
\hline $\mathrm{H}$ & 0.58737471 & 1.48340683 & -2.73779734 \\
\hline $\mathrm{C}$ & 1.58718814 & 2.46309737 & 0.86543956 \\
\hline $\mathrm{H}$ & 1.87888315 & 2.75042764 & 1.87207730 \\
\hline $\mathrm{C}$ & 1.33668132 & 3.05150126 & -1.47165122 \\
\hline $\mathrm{C}$ & 1.68750472 & 3.38240072 & -0.16718390 \\
\hline N & 2.18095090 & 4.72297797 & 0.12044832 \\
\hline O & 2.26795868 & 5.51190821 & -0.80290636 \\
\hline O & 2.48098242 & 4.98998268 & 1.27008542 \\
\hline $\mathrm{C}$ & -1.44384882 & -1.06436219 & 0.45747015 \\
\hline $\mathrm{H}$ & -1.72017409 & -1.55725951 & 1.39002572 \\
\hline C & -2.33330563 & -0.33663597 & -0.22131203 \\
\hline $\mathrm{H}$ & -2.00894190 & 0.15350699 & -1.14048609 \\
\hline $\mathrm{C}$ & -3.71791908 & -0.14214797 & 0.15888933 \\
\hline $\mathrm{C}$ & -4.45528558 & -0.73324192 & 1.15239007 \\
\hline C & -6.06766212 & 0.65999630 & 0.25290294 \\
\hline $\mathrm{H}$ & -4.05105298 & -1.48763496 & 1.82156425 \\
\hline $\mathrm{C}$ & -5.79103638 & -0.27446832 & 1.20648721 \\
\hline $\mathrm{H}$ & -7.00164402 & 1.17428177 & 0.06448753 \\
\hline $\mathrm{H}$ & -6.52497980 & -0.62821051 & 1.92365550 \\
\hline S & -4.69528425 & 0.99483925 & -0.72909789 \\
\hline $\mathrm{H}$ & 1.42935038 & 3.78933646 & -2.26089750 \\
\hline $3 \mathrm{~b}^{+}$ & \multicolumn{3}{|c|}{ IEF-PCM (acetonitrile)/6-311G(d), $\quad-1621.59804454192$} \\
\hline $\mathrm{C}$ & -2.22695844 & 1.60678330 & -0.82091622 \\
\hline $\mathrm{C}$ & -1.93906538 & 2.58575832 & 0.11770514 \\
\hline $\mathrm{C}$ & -2.93358326 & 3.44175193 & 0.54596104 \\
\hline
\end{tabular}




\begin{tabular}{|c|c|c|c|}
\hline $\mathrm{C}$ & -4.21185641 & 3.29144055 & 0.01437818 \\
\hline $\mathrm{C}$ & -4.48341276 & 2.29713778 & -0.91987558 \\
\hline C & -3.48944859 & 1.42865661 & -1.35692495 \\
\hline $\mathrm{C}$ & -0.01582906 & 1.34549682 & -0.36767126 \\
\hline $\mathrm{C}$ & -0.49220477 & 2.48007980 & 0.52215162 \\
\hline $\mathrm{H}$ & -2.72922566 & 4.21688553 & 1.28180656 \\
\hline $\mathrm{H}$ & -5.00894873 & 3.95640909 & 0.33529477 \\
\hline $\mathrm{H}$ & -5.48885017 & 2.19300140 & -1.31803184 \\
\hline $\mathrm{H}$ & -3.71383447 & 0.65266667 & -2.08346442 \\
\hline $\mathrm{N}$ & -1.03955057 & 0.88408829 & -1.08382495 \\
\hline $\mathrm{C}$ & -0.41049202 & 2.05385223 & 1.99504087 \\
\hline $\mathrm{H}$ & 0.62256109 & 1.95666701 & 2.34047856 \\
\hline $\mathrm{H}$ & -0.90422160 & 2.80689140 & 2.61755657 \\
\hline $\mathrm{H}$ & -0.91777734 & 1.09530022 & 2.15547190 \\
\hline $\mathrm{C}$ & 0.24444017 & 3.79406675 & 0.25969029 \\
\hline $\mathrm{H}$ & -0.23543859 & 4.59654245 & 0.82926833 \\
\hline $\mathrm{H}$ & 1.29258054 & 3.75611709 & 0.56770366 \\
\hline $\mathrm{H}$ & 0.20757179 & 4.06348877 & -0.80065890 \\
\hline $\mathrm{C}$ & -0.98265691 & -0.28143845 & -1.96526968 \\
\hline $\mathrm{H}$ & -0.14387197 & -0.16795727 & -2.65514289 \\
\hline $\mathrm{H}$ & -1.88288223 & -0.26652432 & -2.58454314 \\
\hline $\mathrm{C}$ & -0.88290315 & -1.56355556 & -1.18359410 \\
\hline $\mathrm{C}$ & 0.07038465 & -2.53044879 & -1.53460657 \\
\hline $\mathrm{C}$ & 0.16100381 & -3.72723185 & -0.82301387 \\
\hline $\mathrm{H}$ & 0.90648900 & -4.46608427 & -1.10751019 \\
\hline $\mathrm{C}$ & -1.71907098 & -1.80851579 & -0.11033676 \\
\hline $\mathrm{H}$ & -2.46215242 & -1.07624031 & 0.19634818 \\
\hline $\mathrm{C}$ & -0.68173892 & -3.96681182 & 0.24063982 \\
\hline $\mathrm{C}$ & -1.61235167 & -2.99876874 & 0.59136854 \\
\hline $\mathrm{N}$ & -2.49770190 & -3.23459513 & 1.72799178 \\
\hline O & -2.41767660 & -4.30475833 & 2.29973472 \\
\hline O & -3.26759043 & -2.34764591 & 2.04529396 \\
\hline $\mathrm{C}$ & 1.26653345 & 0.77591689 & -0.46862377 \\
\hline $\mathrm{H}$ & 1.39485305 & -0.03533603 & -1.18130796 \\
\hline $\mathrm{C}$ & 2.35443015 & 1.17122646 & 0.25448438 \\
\hline $\mathrm{H}$ & 2.25301160 & 1.98880685 & 0.96549610 \\
\hline $\mathrm{C}$ & 3.64242798 & 0.58761169 & 0.15801475 \\
\hline $\mathrm{C}$ & 4.07361752 & -0.48421826 & -0.60675391 \\
\hline $\mathrm{C}$ & 6.03848399 & 0.08302432 & 0.45658590 \\
\hline $\mathrm{H}$ & 3.42016077 & -1.04927870 & -1.26479582 \\
\hline $\mathrm{C}$ & 5.43284346 & -0.76748482 & -0.43564567 \\
\hline $\mathrm{H}$ & 7.07563064 & 0.08669054 & 0.76781034 \\
\hline $\mathrm{H}$ & 5.95881026 & -1.56775726 & -0.94410230 \\
\hline S & 4.96166488 & 1.23694733 & 1.10137666 \\
\hline $\mathrm{H}$ & -0.62030257 & -4.88987138 & 0.80650468 \\
\hline O & 0.88802766 & -2.23751981 & -2.55803660 \\
\hline $\mathrm{H}$ & 1.51232406 & -2.95461084 & -2.72804238 \\
\hline
\end{tabular}

